\title{
Role of SOX9 in cancer stem cell regulation in lung cancer chemoresistance and particle-induced carcinogenesis
}

\author{
Maria Voronkova \\ mavoronkova@mix.wvu.edu
}

Follow this and additional works at: https://researchrepository.wvu.edu/etd

Part of the Medical Cell Biology Commons

\section{Recommended Citation}

Voronkova, Maria, "Role of SOX9 in cancer stem cell regulation in lung cancer chemoresistance and particle-induced carcinogenesis" (2019). Graduate Theses, Dissertations, and Problem Reports. 4075. https://researchrepository.wvu.edu/etd/4075

This Dissertation is protected by copyright and/or related rights. It has been brought to you by the The Research Repository @ WVU with permission from the rights-holder(s). You are free to use this Dissertation in any way that is permitted by the copyright and related rights legislation that applies to your use. For other uses you must obtain permission from the rights-holder(s) directly, unless additional rights are indicated by a Creative Commons license in the record and/ or on the work itself. This Dissertation has been accepted for inclusion in WVU Graduate Theses, Dissertations, and Problem Reports collection by an authorized administrator of The Research Repository @ WVU.

For more information, please contact researchrepository@mail.wvu.edu. 


\title{
Role of SOX9 in cancer stem cell regulation in lung cancer chemoresistance and particle-induced carcinogenesis
}

\author{
Maria A. Voronkova \\ Dissertation submitted \\ to the School of Medicine \\ at West Virginia University
}

in partial fulfillment of the requirements for the degree of

Doctor of Philosophy in

Cancer Cell Biology

\author{
Karen Martin, Ph.D., Chair \\ J. Michael Ruppert, M.D./Ph.D. \\ Michael Schaller, Ph.D. \\ Ivan Martinez, Ph.D. \\ HanGang Yu, Ph.D. \\ Yon Rojanasakul, Ph.D., Mentor
}

Cancer Cell Biology Program

Morgantown, West Virginia

2019

Keywords: chemoresistance, SOX9, cancer stem cells, NSCLC, ALDH

Copyright 2019 Maria Voronkova 


\title{
Abstract \\ Role of SOX9 in cancer stem cell regulation in lung cancer chemoresistance and particle-induced carcinogenesis
}

\begin{abstract}
Maria A. Voronkova
Lung cancer is a leading cause of cancer-related death worldwide. Every year it claims more lives than other common malignancies, breast, prostate, and colorectal cancers, combined. And despite recent improvements in disease diagnostics and treatment, drug resistance and tumor relapse remain major contributors to low patient survival. Both issues have been largely attributed to the existence of cancer stem-like cells (CSCs) or tumor initiating cells (TICs). CSCs are a small cell population within heterogeneous tumors that is inherently resistant to apoptosis, chemo- and radiation therapy. Thus, it is critical to elucidate CSC-specific signaling pathways and identify early biomarkers for therapeutic intervention. This body of work investigates molecular mechanisms of CSC regulation by an embryonic transcription factor SOX9. We show here that SOX9 is upregulated upon exposure to cisplatin and positively regulates cancer stem-like properties and chemoresistance of non-small cell lung cancer (NSCLC) cells. Moreover, we demonstrate for the first time that the stem-cell marker ALDH1A1 is a direct SOX9 transcriptional target. We also show that SOX9 expression is upregulated in human lung epithelial cells chronically exposed to single-walled carbon nanotubes (SWCNT). SOX9 was identified as a critical factor promoting metastatic and stem-like properties in these cells. We also observed a correlation between SOX9 levels and ALDH expression and activity in this model of carcinogenesis, suggesting that activation of SOX9-ALDH axis may have a broader role in the regulation of cellular stress responses. Taken together, our novel findings on the role of SOX9-ALDH axis support the use of this CSC regulator as a prognostic marker of cancer chemoresistance and as a potential drug target for CSC therapy. The SOX9-ALDH axis could also have a broad role in the regulation of CSC survival under different stress conditions.
\end{abstract}




\section{Acknowledgements}

First and foremost, I would like to thank my advisor Dr. Yon Rojanasakul for being patient and understanding, for pushing me to strive for more, and for supporting through all the ups and downs of research.

Furthermore, I would like to thank my committee members, Dr. Karen Martin, Dr. J. Michael Ruppert, Dr. Michael Schaller, Dr.HanGang Yu, Dr. Ivan Martinez and Dr. Patrick Ma for their dedication, assistance and guidance throughout my PhD projects.

I also want to acknowledge the members of Rojanasakul lab for making therapeutic conversations, putting up with me for so long and providing a very friendly work environment.

I want to thank all CCB students and faculty members for continued encouragement in not giving up and being a better version of myself.

I would like to thank Dr. Kathleen Brundage for her help and encouraging during my flow cytometry experiments.

Finally, I want to thank Yuriy Loskutov who got all this started and pushed me to achieve my goals. 


\section{Table of Contents}

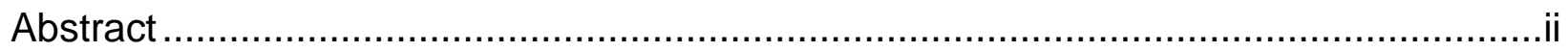

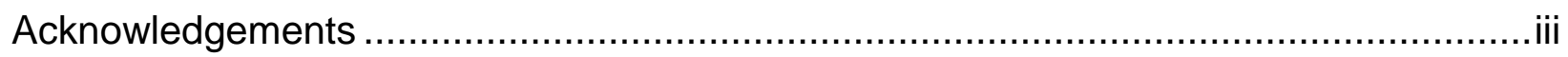

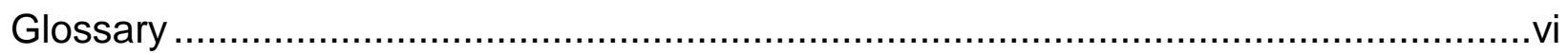

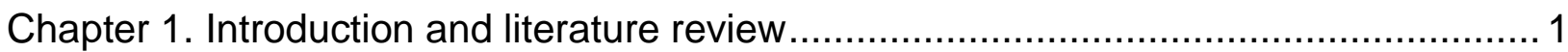

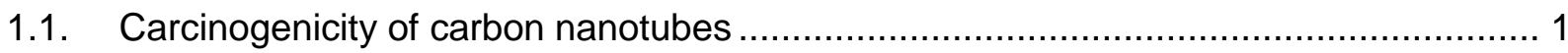

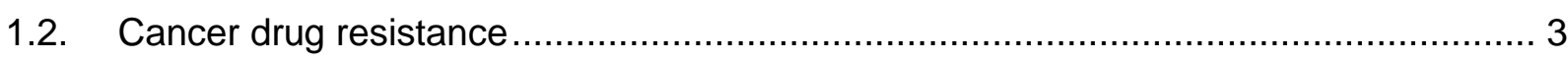

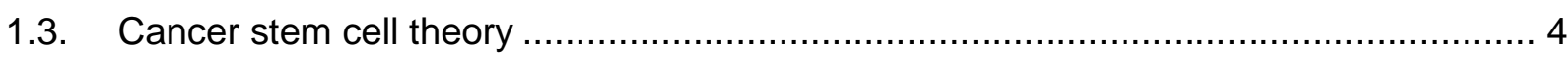

Figure 1. Properties and potential roles of cancer stem cells........................................ 5

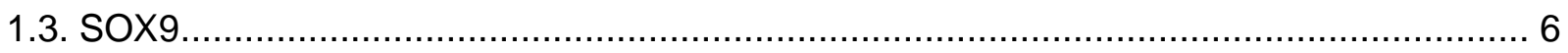

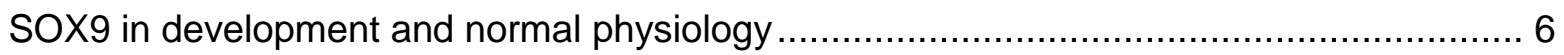

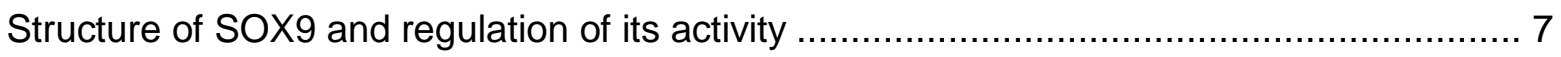

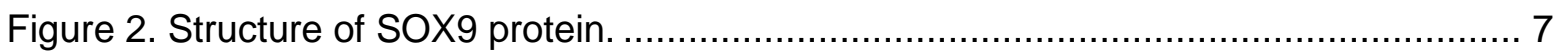

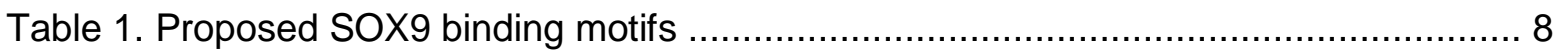

Table 2. Signaling pathways involved in the regulation of SOX9 expression and activity ...10

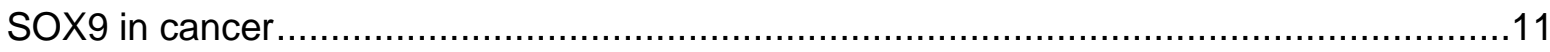

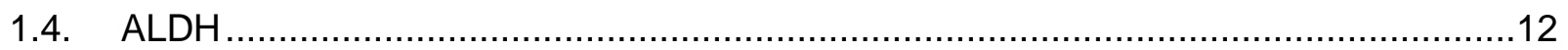

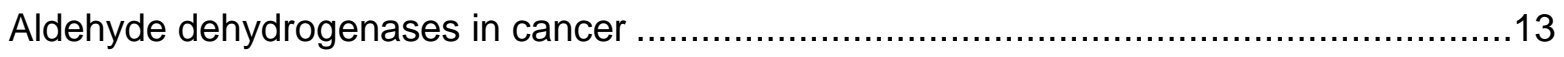

Figure 3. Summary of ALDH1A1 regulation and functions ........................................14

Table 3. Functions of ALDH isoforms other than ALDH1A1 in cancer .............................15

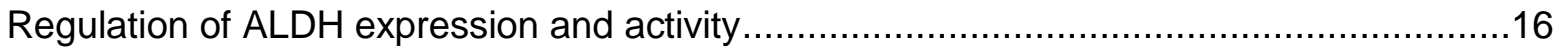

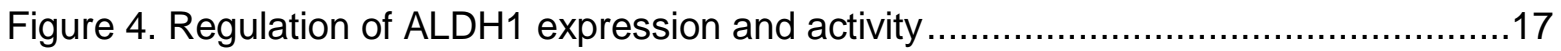

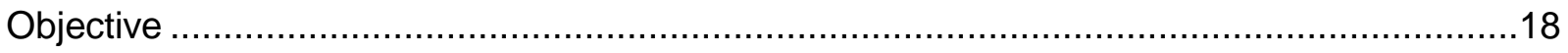

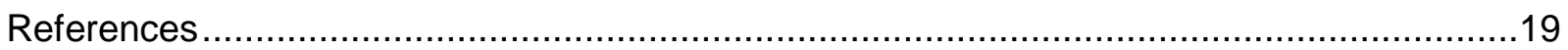

Chapter 2: SOX9-ALDH axis determines resistance to chemotherapy in non-small cell

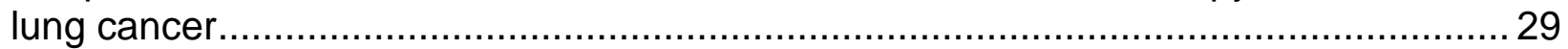

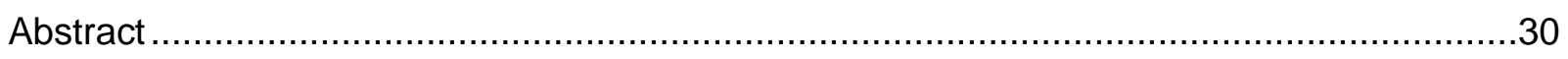

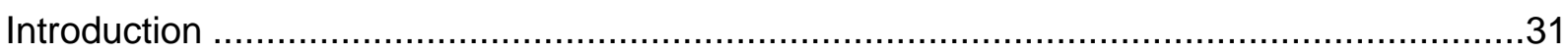

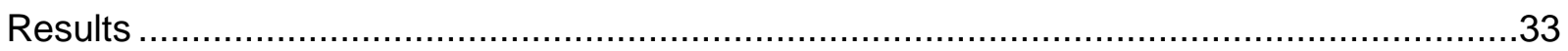

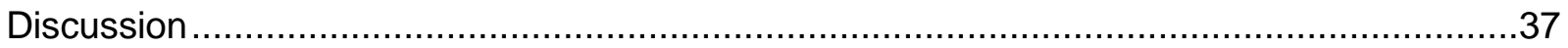

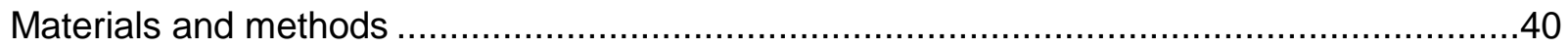

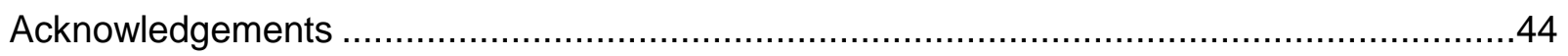

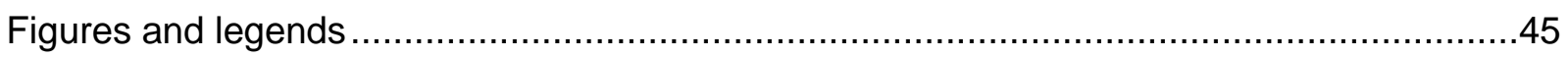

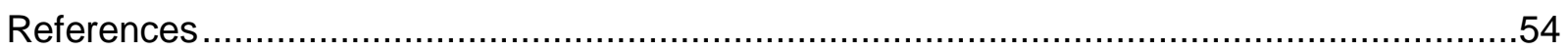

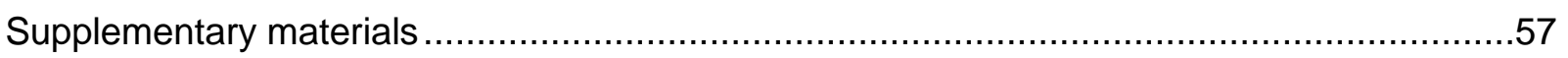


Chapter 3: SOX9 regulates cancer stem-like properties and metastatic potential of

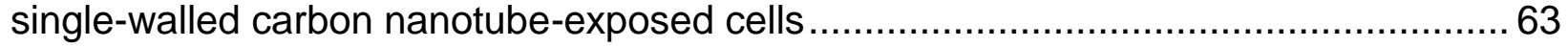

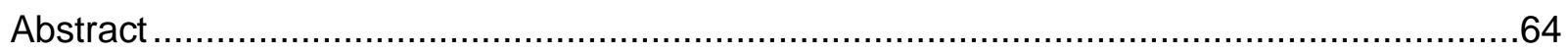

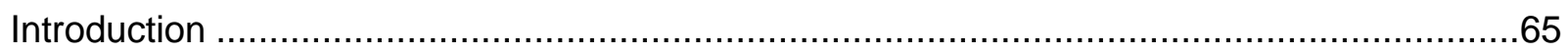

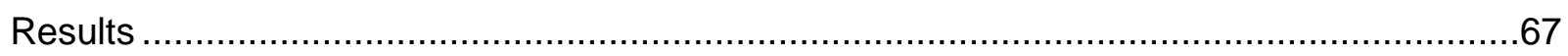

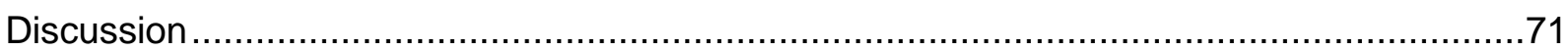

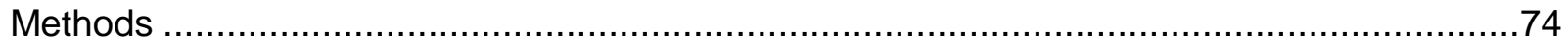

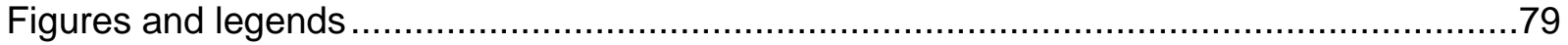

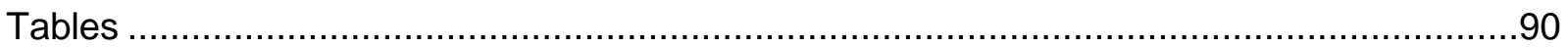

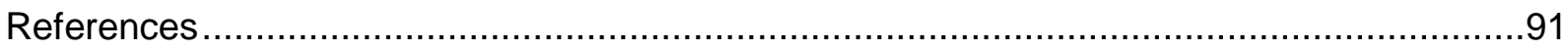

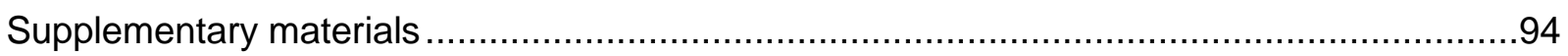

Chapter 4: General discussion and future directions ............................................. 98

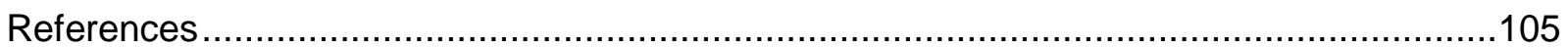




\section{Glossary}

ABCB1 - ATP binding cassette subfamily $B$ member 1

$A B C C 1$ - ATP binding cassette subfamily $C$ member 1

ABCG2 - ATP binding cassette subfamily $G$ member 2

ALDH - aldehyde dehydrogenase

ALK - anaplastic lymphoma receptor tyrosine kinase

ATRA - all-trans retinoic acid

BRAF - serine/threonine-protein kinase B-Raf

CNT - carbon nanotubes

CSCs - cancer stem cells

CTR1 - copper transporter

ECM - extracellular matrix

EGFR - epidermal growth factor receptor

EMT - epithelial-to-mesenchymal transition

GSK3 kinase - glycogen synthase kinase-3

HMG - high mobility group DNA-binding domain

MWCNT - multi-walled carbon nanotubes

NADP+ - nicotinamide adenine dinucleotide phosphate

Nanog - nanog homeobox

NSCLC - Non-small cell lung cancer

Oct4 - octamer-binding protein 4; POU class 5 homeobox 1

PDAC - pancreatic ductal adenocarcinoma

$\mathrm{RA}$ - retinoic acid

ROS - reactive oxygen species

ROS - ROS proto-oncogene 1, receptor tyrosine kinase

SAEC - small airway epithelial cells

SCLC - small cell lung cancer

SOX2 - SRY-box 2

SOX9 - SRY (sex determining region Y) -Box 9

SWCNT - single-walled carbon nanotubes

TAK1 - transforming growth factor beta-activated kinase 1 


\section{Chapter 1. Introduction and literature review}

Lung cancer is the leading cause of cancer-related death worldwide. Non-small cell lung cancer (NSCLC) is the predominant type of lung cancer, accounting for up to $85 \%$ of all lung cancer cases (1). NSCLC is traditionally divided into 3 subtypes based on histological features: adenocarcinoma ( $50 \%$ of cases), squamous cell carcinoma $(\sim 30 \%)$ and large cell carcinoma (2). Despite some recent improvements in the disease management, 5 -year survival rates remain under $20 \%$. Furthermore, over $50 \%$ of all lung cancer cases in the US are diagnosed when the cancer has already metastasized, signifying the need for management of the advanced disease (3). Several types of therapy are available for treatment of NSCLC: surgery, radiation, chemotherapy, targeted-and immunotherapy; however, surgery is usually not an option for later stages (4). Patients who present with stage IV disease undergo molecular testing for driver mutations, such as EGFR and BRAF mutations, ALK and ROS1 rearrangements, and for programmed death ligand-1 (PD-L1) expression (5). Nevertheless, approximately $45 \%$ of patients with NSCLC have unidentified driver mutations $(2,5,6)$, thus platinumbased chemotherapy is commonly used as a main treatment course or in combination with immunotherapy (7). Unfortunately, most patients eventually stop responding to the treatment due to development of drug resistance. As a result, delineating the mechanisms of drug resistance in lung cancer is of critical importance.

\subsection{Carcinogenicity of carbon nanotubes}

Risk factors for lung cancer include exposure to tobacco smoke, genetic predisposition, exposure to ionizing radiation, air pollution and occupational exposure to various carcinogens (8). Engineered nanomaterials have emerged as one of the novel sources for environmental and occupational exposure since they have been increasingly used for various industrial and biomedical applications. Nanomaterials are defined as materials with at least one dimension measuring under $100 \mathrm{~nm}$ (9). Carbon 
nanotubes (CNTs) are one of the most widely used nanomaterials due to their unique properties such as high tensile strength, flexibility, electrical conductivity, and light weight. Common uses of CNTs range from incorporation into rechargeable batteries, automotive parts, and sporting goods to water filters (10). Structurally, they represent a single or multiple two dimensional graphene sheet(s) rolled into a tube with diameter as small as $1 \mathrm{~nm}$ and length from few nanometers to microns.

CNTs exhibit asbestos-like properties, e.g. high aspect ratio (ratio of length to width), durability, and biopersistence; therefore, concerns about their potential carcinogenicity have been mounting. Accumulating evidence suggests that CNT exposure induces genotoxicity and is potentially carcinogenic. Recently, the International Agency for Research on Cancer (IARC) classified one type of multi-walled carbon nanotubes (MWCNTs) as a possible carcinogen while other MWCNTs and single-walled carbon nanotubes (SWCNTs) were considered not classifiable due to insufficient evidence at the present time (11). However, several animal studies demonstrated that CNTs possess carcinogenic properties. For example, several transtracheal intrapulmonary sprayings of MWCNTs were found to cause malignant mesotheliomas, lung adenomas and adenocarcinomas in rats (12). Similar results were observed in a more occupationally and physiologically relevant model, where rats were exposed to MWCNTs via the whole-body inhalation at 6 hours/day, 5 days/week for 2 years. Such exposure caused an increase in lung carcinoma incidences in both male and female animals (13). A number of in vitro studies investigated possible mechanisms of CNT toxicity. Thus, CNTs mimic microtubules and disrupt mitotic spindles causing aneuploidy in lung cells (14). Moreover, SWNCT- or MWCNT-exposed small airway epithelial cells demonstrate a neoplastic-like phenotype that is more aggressive than the asbestos-exposed cells (15). Previous studies by our group have shown that chronic exposure to CNTs induces malignant transformation of human lung epithelial cells (15, 16). These cells exhibit tumorigenic and stem-like properties (17), as well as resistance to chemotherapeutic agents (18). The cells also possess defective apoptosis signaling pathways and abnormal p53 functions have downregulation of the anti-apoptotic genes and destabilized p53 (19). However, metastatic properties of these cells remain largely 
unknown and regulation of stem properties is poorly understood, which is the subject of this investigation.

\subsection{Cancer drug resistance}

Chemotherapy is one of the major treatment options for cancer patients; however, its effectiveness is often hampered by drug resistance. Some tumors are intrinsically resistant to drugs while others acquire resistance during treatment. Chemoresistant cancer cells are generally less responsive to a wide array of therapeutic agents, the phenomenon called multidrug resistance. The molecular mechanisms of chemoresistance are complex and involve several adaptive regulatory pathways. While certain molecular mechanisms are known, the problem of drug resistance remains a major unsolved clinical issue.

An increase in the activity or the expression of drug efflux pumps has been most often attributed to the resistance. The ATP-binding cassette (ABC) transporter family of transmembrane proteins transfers a wide variety of xenobiotics, including most anticancer drugs, and endogenous substrates, out of cells. Multi-drug resistance protein 1 (MDR1, P-glycoprotein or ABCB1), MDR-associated protein 1 (MRP1 or ABCC1) and breast cancer resistance protein (BCRP or $A B C G 2)$ are the main $A B C$ transporter family members implicated in drug resistance, although other $A B C$ transporters may be involved (20). These proteins are overexpressed in multiple cancer types and their expression correlates with drug resistance and patient survival. Unfortunately, inhibitors of $A B C$ transporters have not been successful in clinical trials due to toxicity-related issues and a high redundancy between family members (21). Alterations in cellular drug influx processes can also contribute to cancer drug resistance. For example, cisplatin is actively moved into cells by copper transporter 1 (CTR1) (22) and patients with higher CTR1 levels have better treatment outcomes after a platinum-based therapy (23).

Another common mechanism of drug resistance is drug target alterations, such as mutations or changes in expression levels. For instance, mutations in tubulin are proposed as a resistance mechanism for antimicrotubular agents such as paclitaxel, 
while decreased expression of topoisomerase II promotes cancer cell survival after treatment with topoisomerase II inhibitors. Many chemotherapeutic drugs induce direct or indirect DNA damage that leads to apoptosis if not repaired. Remarkably, cancer cells can modulate DNA damage response pathways, avoiding apoptosis and activating several pro-survival pathways (21).

In summary, multiple mechanisms of cancer chemoresistance have been identified. Intrinsic resistance implies that tumors already have certain characteristics that cause chemotherapy failure while acquired resistance emerges during and after treatment. A number of theories explain how acquired chemoresistance occurs, such as new mutations arising during treatment, cell plasticity leading to adaptation, and a therapy-induced selection of already resistant cells from originally heterogeneous tumors (24).

\subsection{Cancer stem cell theory}

Increasing evidence suggests that cancer stem cells (CSCs) or tumor initiating cells are the main source of tumor dissemination, chemotherapy resistance, and subsequent recurrence $(25,26)$. According to CSC theory, CSCs are a small population of cells sharing certain properties with regular stem cells, such as proliferation, clonogenicity (27), and the ability to self-renew and give rise to all other cells that make up a tumor (28-31). However, these cells are also highly resistant to apoptosis and drugs, and are highly tumorigenic, i.e. a single CSC has the potential to produce a tumor in vivo (32) (Figure 1). Therefore, serial dilutions of cells as well as serial xenotransplantations have been used as a gold standard for proving CSC properties $(33,34)$. Furthermore, CSCs are also termed tumor initiating cells (TIC) due to their ability to produce tumors and metastasize; however, the exact terminology remains a debated topic (24). Another highly discussed theory is the origin of CSCs: do these cells arise from mutated adult stem cells, progenitor cells or from differentiated cells? Moreover, accumulating evidence indicates that CSCs are highly plastic and can reversibly transition between stem and non-stem states (35-37).

CSCs can be characterized and isolated based on a number of stem cell markers. CSC markers appear to be tissue and/or organ specific in general, however, 
CD44, CD133, ALDH1 expression as well as ALDH activity measured by Aldefluor $^{\circledR}$ assay, and ABCG2 drug efflux activity, evaluated by side population assay, are widely used to identify CSCs in solid tumors (25). Moreover, CSCs express multiple embryonic transcription factors, including SOX2, Oct4, and Nanog (38), which have also been used to characterize CSCs. However, each subtype of CSCs isolated and/or characterized based on one set of markers displays a unique phenotype and properties that only partially overlap with other CSC subtypes $(39,40)$. For example, breast CSCs can exist in mesenchymal-like state identified as quiescent and $\mathrm{CD} 24^{-} \mathrm{CD} 44^{+}$and in more proliferative epithelial-like state characterized by high ALDH1 expression and activity (41).
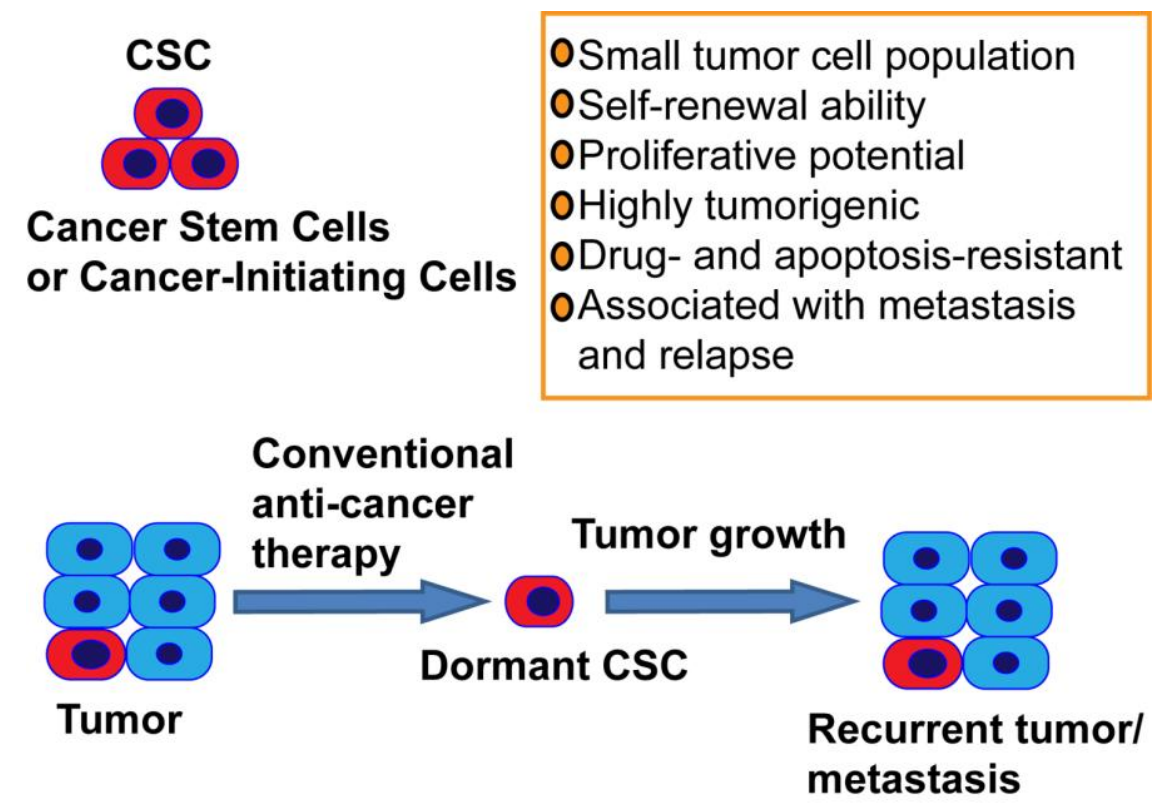

Figure 1. Properties and potential roles of cancer stem cells.

CSCs are inherently more resistant to cytotoxic therapy as compared to regular cancer cells due to many mechanisms, including slower proliferation, high expression of drug efflux pumps, and higher levels of detoxification and DNA repair activities (42-44). Thus, it is not surprising that the conventional chemotherapy kills the bulk of tumor cells but spares CSCs, leading to cancer recurrence (45-47). Multiple attempts have been made to target CSCs: inhibitors of CSC signaling pathways, antibodies against CSC surface markers, inhibitors of epigenetic regulators (e.g. histone deacetylases). 
However, the results are mixed or applicable only to certain tumor types due to high CSC plasticity, similarity to adult stem cells, and limited knowledge on CSC biology (34). Therefore, it is critical to identify specific pathways and mechanisms controlling CSC to successfully eliminate them.

\subsection{SOX9}

\section{SOX9 in development and normal physiology}

Many developmental proteins are now being recognized as oncogenes due to their ability to regulate not only stem cells but also CSCs. SOX9 (SRY (sex determining region Y)-box 9) is a member of the conserved high-mobility group (HMG)-box class of transcription factors. SOX9 works downstream of SRY (sex-determining region $Y$ ) to initiate mammalian male sex determination. Germline SOX9 mutations cause campomelic dysplasia, a severe, generally lethal developmental condition, characterized by cartilage and bone malformations, and common male sex reversal or ambiguous genitalia (48), while heterozygous mice die hours after birth due to multiple skeletal malformations (49). Consistently, SOX9 is essential for chondrocyte proliferation and early differentiation (50). Furthermore, this transcription factor controls critical stages in the development of many organs, including heart, intestine, pancreas, and central nervous system, where it regulates stem cell homeostasis and differentiation. In adults, SOX9 is expressed in stem cells in multiple tissues, including central nervous system, skin, intestine, liver and pancreas $(48,51,52)$. In lungs, SOX9 regulates branching morphogenesis during embryonic development and a response to injuries in adults $(53,54)$. Consistent with its role in embryonic development, SOX9 expression is reactivated during chronic inflammation and tissue damage which may lead to fibrosis in a number of tissues (48). 


\section{Structure of SOX9 and regulation of its activity}

Figure 2. Structure of SOX9 protein.

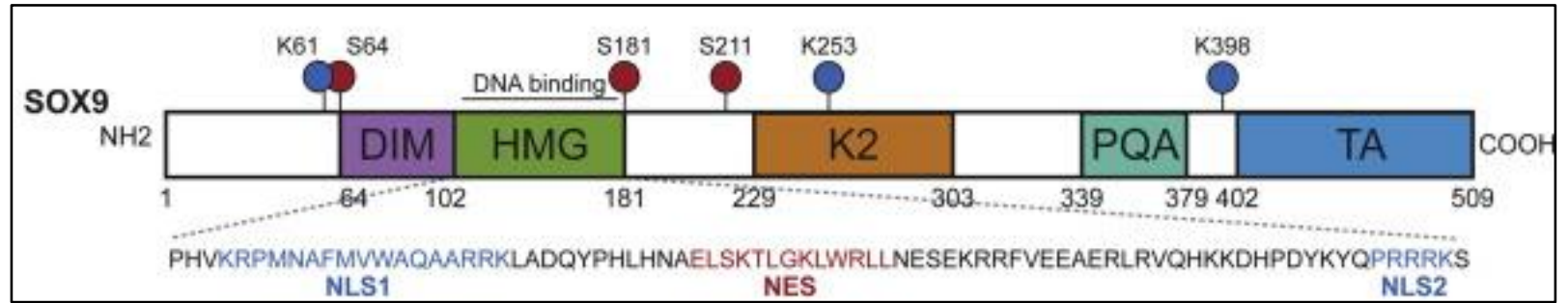

DIM - dimerization domain; HMG - DNA-binding high mobility group domain; K2, TA transactivation domains; NLS - nuclear localization sequences; NES - nuclear export sequence; PQA - proline, glutamine and alanine-rich domain; phosphorylation sites (red), and ubiquitination/sumolyation sites (blue). Reproduced from (48) under the CC BY-NC-ND license (http://creativecommons.org/licenses/by-nc-nd/3.0/).

Structurally, SOX9 contains a DNA-binding high mobility group (HMG) domain, which is highly conserved among SOX proteins, a self-dimerization domain, two transactivation domains, and the proline, glutamine and alanine (PQA) domain, whose function is not known (Figure 2). The HMG domain of SOX9 also contains two nuclear localization sequences and a nuclear export signal to allow for the protein to be shuttled between the nucleus and the cytoplasm (48). SOX9 binds to a consensus DNA sequence, AACAAT, shared with other SOX proteins, while two flanking nucleotides from both sides of this sequence determine the binding specificity of SOX proteins (55). Notably, several recent studies utilizing ChIP-seq assay have identified novel SOX9 binding motifs (Table 1), indicating that SOX9 binding may be tissue specific or context dependent. Moreover, SOX9 can bind its targets as a dimer, which is more common in chondrogenesis, or as a monomer, as proposed in the case of sex differentiation (56) and other non-chondrocyte specific gene targets (57). This transcription factor also cooperates with other SOX family members, SOX5 and SOX6, on promoters of several chondrocyte-specific genes $(58,59)$. Additionally, SOX9 was shown to heterodimerize with SOX8 or SOX10 but not with other SOX proteins in a way involving the DNAbinding domain of one binding partner and the dimerization domain of another (60). A different mode of SOX9 action involves cooperation with a different transcription factor, NF-Y, when SOX9 does not bind to DNA directly but is critical for transcription activation of cell cycle regulatory genes (61). Interestingly, it has recently been suggested that 
SOX9 may regulate alternative splicing and that splicing targets are different from the SOX9 transcriptional targets (62).

Table 1. Proposed SOX9 binding motifs

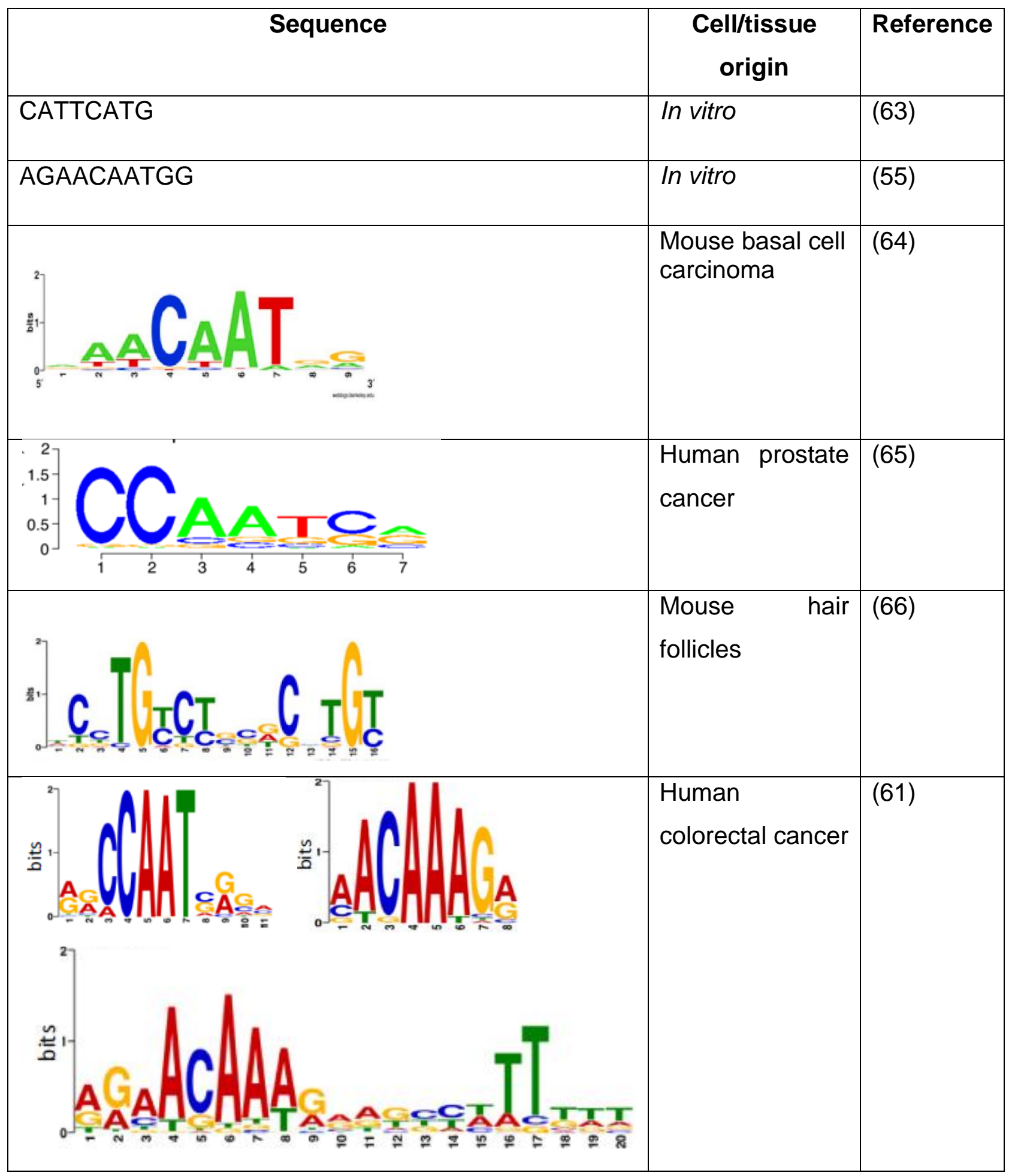


Limited information is available about the direct transcriptional targets of SOX9. The transcription factor can work as a transcriptional activator or repressor, depending on cellular context. For instance, SOX9 cooperates with Gli proteins to repress gene transcription of Col10a1 and to inhibit chondrocyte maturation but forms a complex with Sox5/6 to activate the transcription of Col2a1, which is involved in extracellular matrix (ECM) deposition and chondrocyte differentiation (58, 67). SOX9 regulates the expression of stemness genes TCF3, TCF4 and HMGA2 in basal cell carcinoma (64) and was proposed to regulate cell proliferation and senescence by activating p21(cip1), an inhibitor of cell cycle progression $(68,69)$. Conversely, SOX9 is recruited by NF-Y to promoters of cell cycle regulatory genes, such as cyclin B1 (CCNB1), cyclin B2 (CCNB2), cyclin dependent kinase 1 (CDK1), and topoisomerase II (TOP2A), and is critical for expression of these proteins in colorectal cancer (61).

Several signaling pathways leading to SOX9 activation have been identified in developmental biology, such as Sonic hedgehog, Notch, Wnt and others (48, 70). Accumulating evidence also suggests that similar pathways are involved in SOX9 overexpression during carcinogenesis (summarized in Table 2). Other studies showed that SOX9 can be phosphorylated by GSK3 kinase, targeting SOX9 for subsequent ubiquitination and proteasomal degradation $(71,72)$. In addition, SOX9 forms an autoregulatory positive feedback loop using its far upstream (-70kb) enhancer (73). 
Table 2. Signaling pathways involved in the regulation of SOX9 expression and activity

\begin{tabular}{|c|c|c|c|}
\hline Pathway & Organ/model & Mechanism & Reference \\
\hline $\begin{array}{c}\mathrm{PI3K} / \mathrm{AKT} / \\
\mathrm{mTOR} / \mathrm{GSK} 3\end{array}$ & $\begin{array}{l}\text { Medulloblastoma, various } \\
\text { cancer cell lines }\end{array}$ & Ubiquitination and degradation & (72), (71) \\
\hline Notch & $\begin{array}{l}\text { Esophageal } \\
\text { adenocarcinoma, lung } \\
\text { adenocarcinoma }\end{array}$ & $\begin{array}{l}\text { Transcriptional activation by } \\
\text { Notch1 }\end{array}$ & $(74),(75)$ \\
\hline Hedgehog & $\begin{array}{l}\text { Human chondrocytes, } \\
\text { mouse trachea }\end{array}$ & $\begin{array}{l}\text { Transcriptional activation by } \\
\text { Gli1 }\end{array}$ & (76), (77) \\
\hline \multirow{2}{*}{ Wnt } & Mouse chondrocytes & Repression by $\beta$-catenin & $(78)$ \\
\hline & $\begin{array}{l}\text { Human colon cancer, } \\
\text { mouse intestine }\end{array}$ & $\begin{array}{l}\text { Activation by unknown } \\
\text { mechanism }\end{array}$ & (79) \\
\hline Wnt, BMP & Chick embryos & $\begin{array}{l}\text { Phosphorylation leading to } \\
\text { SUMOylation activate } \\
\text { interactions with Slug }\end{array}$ & (80) \\
\hline \multirow[t]{2}{*}{ TGF $\beta$} & $\begin{array}{l}\text { Mouse chondrogenic cell } \\
\text { line, human } \\
\text { chondrosarcoma cell line }\end{array}$ & $\begin{array}{l}\text { Phosphorylation and } \\
\text { stabilization by p38 and } \\
\text { Smad2/3, stabilization at } \\
\text { transcription site by Smad2/3 }\end{array}$ & $\begin{array}{l}(81) \\
(82)\end{array}$ \\
\hline & Rat kidney fibroblasts & $\begin{array}{l}\text { Transcriptional activation by } \\
\text { Smad3 }\end{array}$ & (83) \\
\hline Kras & $\begin{array}{l}\text { Human pancreatic } \\
\text { cancer }\end{array}$ & $\begin{array}{l}\text { Increased expression through } \\
\text { NF-kB pathway, nuclear } \\
\text { localization }\end{array}$ & (84) \\
\hline$N F-k B$ & $\begin{array}{l}\text { Human pancreatic } \\
\text { cancer }\end{array}$ & $\begin{array}{l}\text { Transcriptional activation by } \\
\text { p65 }\end{array}$ & (85) \\
\hline Hippo & $\begin{array}{l}\text { Human esophageal } \\
\text { cancer }\end{array}$ & $\begin{array}{l}\text { Transcriptional activation by } \\
\text { YAP1 }\end{array}$ & (86) \\
\hline FGF & $\begin{array}{l}\text { Mouse primary } \\
\text { chondrocytes }\end{array}$ & $\begin{array}{l}\text { Activated through MAPK } \\
\text { pathway }\end{array}$ & (87) \\
\hline cAMP & $\begin{array}{l}\text { Rat chondrosarcoma, in } \\
\text { vitro }\end{array}$ & $\begin{array}{l}\text { Increased DNA binding after } \\
\text { phosphorylation by PKA }\end{array}$ & (88) \\
\hline
\end{tabular}




\section{SOX9 in cancer}

SOX9 has been implicated in the formation and progression of multiple cancer types (89). It is overexpressed in various solid tumors, such as breast, prostate, pancreatic and lung cancers. Furthermore, SOX9 overexpression positively correlates with poor overall and disease-free survival in these malignancies $(90,91)$. Interestingly, contradictory results on the prognostic role of SOX9 overexpression have been reported in melanoma $(92,93)$. However, a study by Yang et al. showed that low SOX9 expression restricts metastasis while its high expression promotes it (94). Similar dosedependent action was proposed for SOX9 in colorectal cancer (95), explaining contradictory reports on tumor suppressing and oncogenic properties of this transcription factor. For instance, high SOX9 levels predict low risk of relapse in stage II colon cancer patients (96) and inhibits proliferation of colorectal cell lines (97). In contrast, SOX9 promotes invasion, migration and tumor formation in colorectal cells and correlates with lower 5-year survival in patients according to other studies $(98,99)$. An integrating model, proposed by Prevostel et al., suggests that there is a critical level of SOX9 required for optimal proliferation of colorectal cells while too low or too high SOX9 expression would inhibit cell growth (97).

SOX9 promotes acinar-to-ductal reprogramming and is required for oncogenic $\mathrm{Kras}^{\mathrm{G} 12 \mathrm{D}}$-mediated induction of premalignant lesions in pancreatic ductal adenocarcinoma (PDAC) (100). Kras, in turn, induces SOX9 expression, protein stability, nuclear translocation, and activating phosphorylation. SOX9 levels are increased via TAK1-kinase and may form a positive feedback loop with NF-KB pathway in PDAC (84). Moreover, NF-kB subunit p65 can directly regulate transcription from the SOX9 promoter (85). SOX9 has also been recently identified as a part of the $\mathrm{Wnt} / \beta$ catenin-related signature in driver gene-negative (for known driver mutations) lung adenocarcinoma. Patients identified as a high-risk cohort, defined by high SOX9, $\beta$ catenin, DVL3 and low Wnt2b expression, had a substantial decrease in overall survival. The difference in overall survival was more striking for stage 1 and 2 carcinomas (101). 
Other studies demonstrated that SOX9 promotes cancer stem-like properties in multiple tumor types. SOX9 has been shown to stimulate self-renewal and to inhibit cell differentiation in basal cell carcinoma (64). This transcription factor is also highly expressed in liver CSCs and controls self-renewal and symmetrical cell division, promoting tumorigenicity in hepatocellular carcinoma (102). It also cooperates with the master - regulator of epithelial-to-mesenchymal transition (EMT) Slug to maintain normal mammary stem cells and breast CSCs, and to reprogram differentiated breast cells into stem cells (103). In lungs, SOX9 expression is associated with stemness of lung carcinoma (104), although the underlying mechanisms are not known. SOX9 was shown to promote invasion in pancreatic cancer (85).

A few reports suggest that SOX9 may enhance chemoresistance; however, the exact molecular mechanisms and downstream effectors remain unknown. For instance, Santos et al. (105) observed a correlation between SOX9 expression and a response to cisplatin in gastric cancer cells. These results were supported by knockdown and overexpression experiments in this model and in medulloblastoma (72). Consistent with preclinical studies, SOX9 overexpression in colorectal cancer correlated with poor survival in 5-FU treated patients but not in untreated patients (106). Strong SOX9 expression in breast cancer after chemotherapy was associated with short overall survival (107). While the association between SOX9 expression and chemotherapeutic efficacy has been reported, the underlying mechanisms of SOX9-mediated chemoresistance remain largely unknown.

\subsection{ALDH}

The aldehyde dehydrogenase (ALDH) superfamily of $\mathrm{NADP}^{+}$-dependent enzymes includes 19 members in humans and catalyzes the oxidation of various aldehydes into corresponding carboxylic acids. The enzymes detoxify endogenous aldehydes, generated during normal cellular metabolism, as well as exogenous aldehydes, generated from the exposure to environmental agents and drugs (108). Most isoforms are widely distributed throughout the body and can be localized to the cytosol, mitochondria, peroxisomes and/or nucleus. While individual ALDH enzymes have preferred substrates, certain cross-reactivity has been observed $(108,109)$. 
It is worth noting a number of discrepancies regarding ALDH nomenclature in the literature. Thus, the term ALDH1 or ALDH could be utilized to define any member of the ALDH1 subfamily (e.g. ALDH1A1, 1A2, 1A3, 1B1, 1L1, 1L2) but often used to describe ALDH1A1 specifically (110). Another controversy arises with respect to the standard method to evaluate ALDH activity, Aldefluor $^{\circledR}$ assay. The assay is based on the fluorescent ALDH substrate, BODIPY-amino acetaldehyde (BAAA), which passively diffuses into cells but is retained intracellularly after being converted into a negatively charged product by ALDH. This assay was first employed to isolate hematopoietic stem cells (111) but is currently used to characterize and isolate both normal and CSCs. The Aldefluor $^{\circledR}$ assay was considered ALDH1A1 specific, but recent reports suggest that it detects the activity of several or predominantly expressed organ- and tissue-specific ALDH isoforms $(112,113)$.

\section{Aldehyde dehydrogenases in cancer}

While all group members participate in the detoxification of endogenous and exogenous agents, ALDH1A1 is particularly known as a CSC marker in multiple cancer types (114). Isolated ALDH ${ }^{\text {high }}$ cells are extremely tumorigenic and display an increased ability to colonize distant organs (115). Moreover, ALDH1A1 is overexpressed in many cancer types and usually correlates with poor prognosis. For example, high ALDH1 expression correlates with poor overall survival in ovarian cancer (116). While individual reports do not agree on the prognostic value of ALDH1A1 overexpression, metaanalysis studies demonstrate a strong correlation between ALDH1 expression and overall and disease-free survival in lung cancer $(117,118)$, colorectal cancer (119), and head and neck squamous cell carcinomas (120).

ALDH1A1 was shown to regulate cell proliferation and drug resistance (109, 121). Moreover, elevated ALDH1 expression after neoadjuvant chemotherapy is associated with poor response and early relapse in ovarian, breast and esophageal cancers (122-124). ALDH1A1 is also overexpressed in many drug-resistant cell lines (125-127), suggesting that it plays a role in chemoresistance.

In addition to serving as a stem cell marker, ALDH governs CSC differentiation by producing retinoic acid (128). ALDH1A1, ALDH1A2, ALDH1A3, and ALDH8A1 
convert retinaldehyde into retinoic acid (RA). Retinoic acid binds to its nuclear receptor followed by attachment to retinoic acid response elements on the promoter of target genes and induces stem cell differentiation (129). Remarkably, all-trans retinoic acid (ATRA) has been successfully used in the treatment of acute promyelocytic leukemia (APL) in combination with chemotherapy but was not effective for other tumor types $(130,131)$.

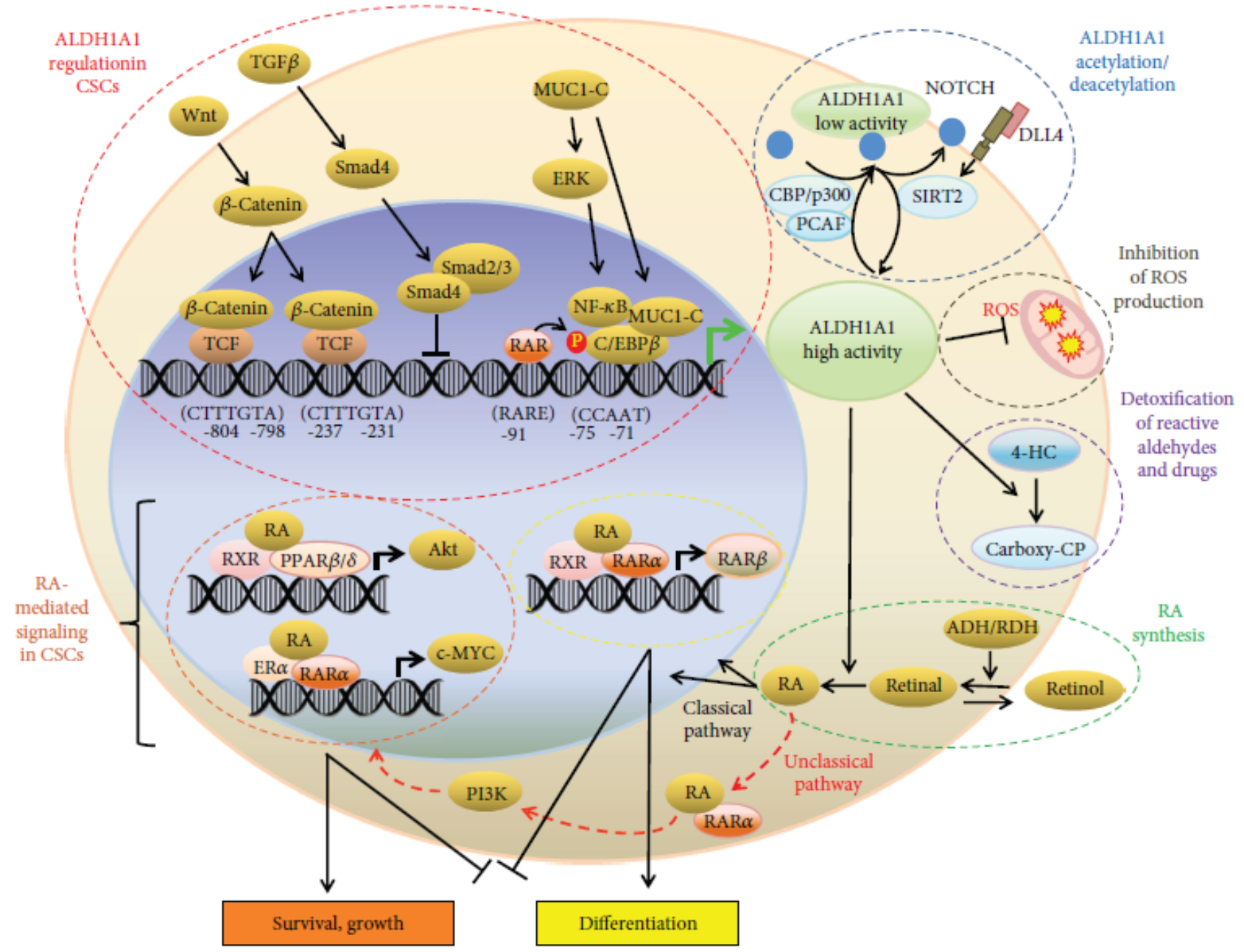

Figure 3. Summary of ALDH1A1 regulation and functions

Reproduced from (132) under the Creative Commons Attribution License.

High ALDH activity, which is typical for stem cells, could also support CSC survival (Figure 3). For instance, ALDH1A1 can detoxify an active derivate of 
cyclophosphamide, 4-hydroxycyclophosphamide (4-HC), and therefore directly protect cancer cells from the drug $(133,134)$. A recent study also reported that $A L D H^{\text {high }}$ cells exhibit low ROS levels relative to ALDH ${ }^{\text {low }}$ counterparts (135). In addition, ALDH inhibition leads to an accumulation of toxic ROS in targeted-therapy selected CSCs, suggesting that the high ALDH activity is vital for CSC survival (136). NADH production by ALDH enzymes has also been proposed as a source of increased ATP synthesis and therefore cancer cell survival in NSCLC (137). Furthermore, ALDH1A1 stimulates the activity of NRF2, a key regulator of cellular antioxidant response (138).

It becomes evident that not only ALDH1A1 but other ALDH isoforms contribute to the malignant properties and to chemotherapy resistance of CSCs (Table 3). For example, high ALDH3A1 and ALDH1A3 expression is associated with lung cancer progression $(139,140)$. Moreover, ALDH1A3 is the main isoform responsible for the increased ALDH activity in breast cancer (141). Interestingly, while ALDH1A3 contributes to the Aldefluor ${ }^{\circledR}$ activity in breast cancer, ALDH1A1 controls the metastatic and drug resistance properties of the cells (142), suggesting a potential functional cooperation between the isoforms.

Table 3. Functions of ALDH isoforms other than ALDH1A1 in cancer

\begin{tabular}{|c|c|c|}
\hline Isoform & Experimental evidence & Reference \\
\hline ALDH1A2 & $\begin{array}{l}\text { RA production, knockdown induces sensitivity to 1- } \beta \text {-D- } \\
\text { arabinofuranosylcytosine (AraC) in leukemic cells, } \\
\text { overexpression induces cell proliferation, higher } \\
\text { clonogenicity and resistance to doxorubicin and 4-HC in } \\
\text { leukemic and NSCLC cells }\end{array}$ & $\begin{array}{l}(143), \\
(144), \\
(145)\end{array}$ \\
\hline ALDH1A3 & $\begin{array}{l}\text { RA production, increases survival and drug resistance in } \\
\text { mesothelioma, enhances growth of glioma CSCs. } \\
\text { ALDH1A3 knockout decreases clonogenicity of } \\
\text { neuroblastoma CSCs, ALDH1A3 knockdown induces } \\
\text { apoptosis and inhibits xenograft growth in melanoma, } \\
\text { inhibits clonogenicity and tumor formation in in vivo } \\
\text { limited dilutions in NSCLC cells. }\end{array}$ & $\begin{array}{l}(146), \\
(147), \\
(148), \\
(149), \\
(150), \\
(140)\end{array}$ \\
\hline ALDH1B1 & $\begin{array}{l}\text { Knockdown inhibits clonogenicity and tumor growth in } \\
\text { vivo in colorectal cells }\end{array}$ & $(151)$ \\
\hline
\end{tabular}




\begin{tabular}{|c|c|c|}
\hline ALDH1L1 & $\begin{array}{l}\text { Overexpression in multiple cancer cell lines inhibits cell } \\
\text { proliferation }\end{array}$ & $(152)$ \\
\hline ALDH1L2 & $\begin{array}{l}\text { Knockdown inhibits metastases but not primary tumor } \\
\text { growth in melanoma xenografts }\end{array}$ & $(153)$ \\
\hline ALDH2 & $\begin{array}{l}\text { Overexpression induces cell proliferation, higher } \\
\text { clonogenicity and resistance to doxorubicin and } 4-\mathrm{HC} \text { in } \\
\text { leukemic and NSCLC cells }\end{array}$ & $(145)$ \\
\hline ALDH3A1 & $\begin{array}{l}\text { Knockdown increases sensitivity to } 4-\mathrm{HC} \text { in NSCLC } \\
\text { cells, pharmacological inhibitions sensitize NSCLC and } \\
\text { glioblastoma cells to mafosfamide }\end{array}$ & $\begin{array}{l}(154), \\
(155)\end{array}$ \\
\hline ALDH3A2 & $\begin{array}{l}\text { Overexpressed in ovarian tumors versus normal } \\
\text { ovarian tissues }\end{array}$ & $(156)$ \\
\hline ALDH3B1 & $\begin{array}{l}\text { Overexpression in HEK293 cells protects against } \\
\text { oxidative stress }\end{array}$ & $(157)$ \\
\hline ALDH3B2 & Functions unknown & $(109)$ \\
\hline ALDH4A1 & $\begin{array}{l}\text { Overexpression in NSCLC cells reduces ROS under } \\
\text { cellular stress and increases resistance to hydrogen } \\
\text { peroxide }\end{array}$ & $(158)$ \\
\hline ALDH5A1 & $\begin{array}{l}\text { Pharmacological inhibition inhibits cell proliferation in } \\
\text { breast cancer cells in 3D culture }\end{array}$ & $(159)$ \\
\hline ALDH6A1 & $\begin{array}{l}\text { RA production in normal breast cells but not in cancer } \\
\text { cells }\end{array}$ & $(160)$ \\
\hline ALDH7A1 & $\begin{array}{l}\text { Knockdown decreases metastasis and CSC population } \\
\text { in prostate cancer }\end{array}$ & $(161)$ \\
\hline ALDH8A1 & RA production & $(108)$ \\
\hline ALDH9A1 & $\begin{array}{l}\text { No known functions in cancer, involved in GABA } \\
\text { biosynthesis }\end{array}$ & $(108)$ \\
\hline ALDH16A1 & Functions unknown & $(109)$ \\
\hline ALDH18A1 & $\begin{array}{l}\text { Highly expressed in breast cancer cells sensitive to } \\
\text { glutaminase inhibitor }\end{array}$ & $(162)$ \\
\hline
\end{tabular}

\section{Regulation of ALDH expression and activity}

Limited information about ALDH regulation in CSCs is available, and most studies to date have focused on ALDH1A1 isoform (Figures 3 and 4). ALDH1A1 transcription is induced by Mucin 1 in cooperation with CCAAT/enhancer-binding protein 
$\beta$ (C/EBP $\beta)$ transcription factor (163) and by Wnt pathway through $\beta$-catenin (164). However, high levels of retinoic acid downregulate C/EBP $\beta$ and therefore create a negative feedback loop with ALDH1 (165). Interestingly, contradictory results on the role of TGF $\beta$ signaling in ALDH1A1 regulation suggest cell context-dependent functions. For example, Hoshino et al. reported transcriptional repression of ALDH1A1 by Smad4 (166) in pancreatic cancer, while a treatment with TGF- $\beta 1$ induced the number of ALDH-positive cells and ALDH1 expression in cholangiocarcinoma (167) and ALDHpositive population in breast cancer (168). Moreover, TGF- $\beta 2$ treatment decreased $\mathrm{ALDH}^{\text {high }}$ population in clear-cell renal cell carcinoma (169), suggesting a relationship between TGF $\beta$ signaling and ALDH expression which requires further investigations.

Figure 4. Regulation of ALDH1 expression and activity

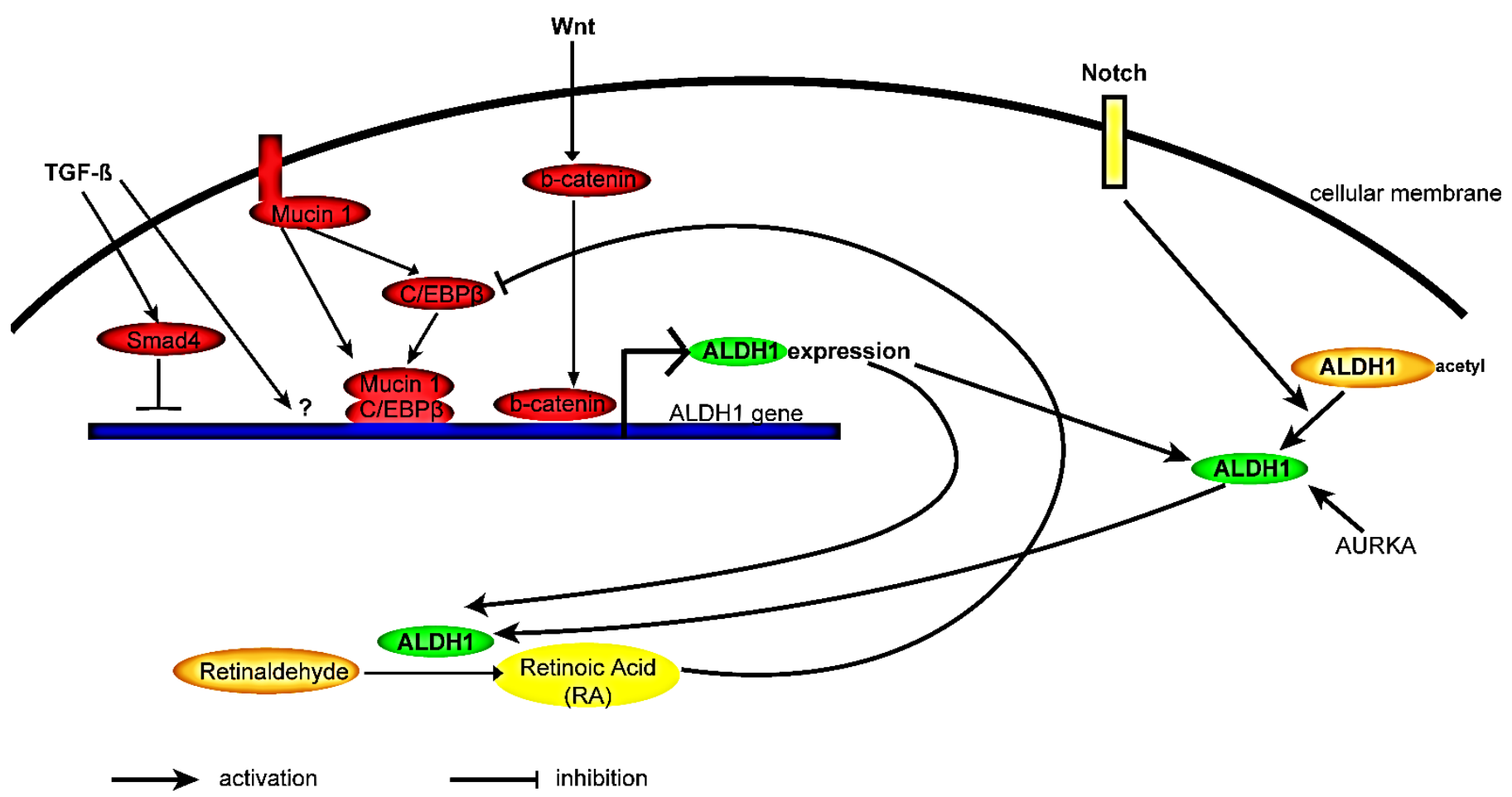

ALDH1A1 activity can be regulated by post-translational modifications. For instance, it is induced by Notch signaling through deacetylation of the enzyme (170). Furthermore, phosphorylation by Aurora kinase A (AURKA), a protein essential for mitosis, increases ALDH1A1 activity and protein stability (171). 


\section{Objective}

Lung cancer is the leading cause of cancer-related mortality with platinum-based chemotherapy commonly used as a main treatment course. Unfortunately, most patients eventually stop responding to the treatment due to development of drug resistance. And while certain molecular mechanisms are known, the problem of drug resistance remains a major unsolved clinical issue. It has been noticed that the conventional chemotherapy spares a population of cancer cells, displaying stem-like properties, which are called cancer stem cells or tumor-initiating cells. Since these cells are generally resistant to chemotherapy and radiation, they eventually give rise to recurrent tumors; hence there is a pressing need to identify markers and potential drug targets, specific to this cancer cell population. Our work is focused on understanding the role of SOX9 in lung CSC regulation and elucidating the underlying mechanisms. SOX9 have been shown to regulate CSCs in several types of cancer but the exact mechanisms and downstream targets are largely unknown. Moreover, there is little information on role of SOX9 in response to chemotherapy. In this work, we sought to determine the functional role of ALDH1A1 as a direct transcriptional target of SOX9 and as a key mediator of druginduced resistance and CSC survival in lung cancer cells. 


\section{References}

1. American Cancer Society. Cancer Facts \& Figures 2019. 2019.

2. Chan BA, Hughes BG. Targeted therapy for non-small cell lung cancer: current standards and the promise of the future. Transl Lung Cancer Res. 2015;4(1):36-54.

3. Cronin KA, Lake AJ, Scott S, Sherman RL, Noone AM, Howlader N, et al. Annual Report to the Nation on the Status of Cancer, part I: National cancer statistics. Cancer. 2018;124(13):2785-800.

4. Postmus PE, Kerr KM, Oudkerk M, Senan S, Waller DA, Vansteenkiste J, et al. Early and locally advanced non-small-cell lung cancer (NSCLC): ESMO Clinical Practice Guidelines for diagnosis, treatment and follow-up. Annals of oncology : official journal of the European Society for Medical Oncology. 2017;28(suppl_4):iv1-iv21.

5. Riely GL. What, When, and How of Biomarker Testing in Non-Small Cell Lung Cancer. Journal of the National Comprehensive Cancer Network : JNCCN. 2017;15(5S):686-8.

6. Cheng L, Alexander RE, Maclennan GT, Cummings OW, Montironi R, Lopez-Beltran A, et al. Molecular pathology of lung cancer: key to personalized medicine. Mod Pathol. 2012;25(3):347-69.

7. Planchard D, Popat S, Kerr K, Novello S, Smit EF, Faivre-Finn C, et al. Metastatic nonsmall cell lung cancer: ESMO Clinical Practice Guidelines for diagnosis, treatment and followup. Annals of oncology : official journal of the European Society for Medical Oncology. 2018;29(Supplement_4):iv192-iv237.

8. Malhotra J, Malvezzi M, Negri E, La Vecchia C, Boffetta P. Risk factors for lung cancer worldwide. Eur Respir J. 2016;48(3):889-902.

9. Boverhof DR, Bramante CM, Butala JH, Clancy SF, Lafranconi M, West J, et al. Comparative assessment of nanomaterial definitions and safety evaluation considerations. Regul Toxicol Pharmacol. 2015;73(1):137-50.

10. De Volder MF, Tawfick SH, Baughman $\mathrm{RH}$, Hart AJ. Carbon nanotubes: present and future commercial applications. Science. 2013;339(6119):535-9.

11. Grosse Y, Loomis D, Guyton KZ, Lauby-Secretan B, El Ghissassi F, Bouvard V, et al. Carcinogenicity of fluoro-edenite, silicon carbide fibres and whiskers, and carbon nanotubes. The Lancet Oncology. 2014;15(13):1427-8.

12. Suzui M, Futakuchi M, Fukamachi K, Numano T, Abdelgied M, Takahashi S, et al. Multiwalled carbon nanotubes intratracheally instilled into the rat lung induce development of pleural malignant mesothelioma and lung tumors. Cancer Sci. 2016;107(7):924-35.

13. Kasai T, Umeda Y, Ohnishi M, Mine T, Kondo H, Takeuchi T, et al. Lung carcinogenicity of inhaled multi-walled carbon nanotube in rats. Part Fibre Toxicol. 2016;13(1):53.

14. Sargent LM, Hubbs AF, Young SH, Kashon ML, Dinu CZ, Salisbury JL, et al. Singlewalled carbon nanotube-induced mitotic disruption. Mutat Res. 2012;745(1-2):28-37.

15. Wang L, Stueckle TA, Mishra A, Derk R, Meighan T, Castranova V, et al. Neoplastic-like transformation effect of single-walled and multi-walled carbon nanotubes compared to asbestos on human lung small airway epithelial cells. Nanotoxicology. 2014;8(5):485-507.

16. Wang L, Luanpitpong S, Castranova V, Tse W, Lu Y, Pongrakhananon V, et al. Carbon nanotubes induce malignant transformation and tumorigenesis of human lung epithelial cells. Nano Lett. 2011;11(7):2796-803.

17. Luanpitpong S, Wang L, Castranova V, Rojanasakul Y. Induction of stem-like cells with malignant properties by chronic exposure of human lung epithelial cells to single-walled carbon nanotubes. Part Fibre Toxicol. 2014;11:22.

18. Pongrakhananon V, Luanpitpong S, Stueckle TA, Wang L, Nimmannit U, Rojanasakul Y. Carbon nanotubes induce apoptosis resistance of human lung epithelial cells through FLICEinhibitory protein. Toxicol Sci. 2015;143(2):499-511. 
19. Chen D, Stueckle TA, Luanpitpong S, Rojanasakul Y, Lu Y, Wang L. Gene expression profile of human lung epithelial cells chronically exposed to single-walled carbon nanotubes. Nanoscale Res Lett. 2015;10:12.

20. Robey RW, Pluchino KM, Hall MD, Fojo AT, Bates SE, Gottesman MM. Revisiting the role of $A B C$ transporters in multidrug-resistant cancer. Nat Rev Cancer. 2018;18(7):452-64.

21. Holohan C, Van Schaeybroeck S, Longley DB, Johnston PG. Cancer drug resistance: an evolving paradigm. Nat Rev Cancer. 2013;13(10):714-26.

22. Song I-S, Savaraj N, Siddik ZH, Liu P, Wei Y, Wu CJ, et al. Role of human copper transporter Ctr1 in the transport of platinum-based antitumor agents in cisplatin-sensitive and cisplatin-resistant cells. Mol Cancer Ther. 2004;3(12):1543-9.

23. Sun S, Cai J, Yang Q, Zhao S, Wang Z. The association between copper transporters and the prognosis of cancer patients undergoing chemotherapy: a meta-analysis of literatures and datasets. Oncotarget. 2017;8(9):16036-51.

24. Kreso A, Dick JE. Evolution of the Cancer Stem Cell Model. Cell Stem Cell. 2014;14(3):275-91.

25. Pattabiraman DR, Weinberg RA. Tackling the cancer stem cells - what challenges do they pose? Nat Rev Drug Discov. 2014;13(7):497-512.

26. Medema JP. Cancer stem cells: The challenges ahead. Nat Cell Biol. 2013;15(4):338-

44.

27. Reya T, Morrison SJ, Clarke MF, Weissman IL. Stem cells, cancer, and cancer stem cells. Nature. 2001;414(6859):105-11.

28. Bonnet D, Dick JE. Human acute myeloid leukemia is organized as a hierarchy that originates from a primitive hematopoietic cell. Nat Med. 1997;3(7):730-7.

29. Lapidot T, Sirard C, Vormoor J, Murdoch B, Hoang T, Caceres-Cortes J, et al. A cell initiating human acute myeloid leukaemia after transplantation into SCID mice. Nature. 1994;367(6464):645-8.

30. Singh SK, Clarke ID, Terasaki M, Bonn VE, Hawkins C, Squire J, et al. Identification of a cancer stem cell in human brain tumors. Neurosurgery. 2003;53(2):487-8.

31. Al-Hajj M, Wicha MS, Benito-Hernandez A, Morrison SJ, Clarke MF. Prospective identification of tumorigenic breast cancer cells. Proc Natl Acad Sci USA. 2003;100(7):3983-8.

32. Quintana E, Shackleton M, Sabel MS, Fullen DR, Johnson TM, Morrison SJ. Efficient tumour formation by single human melanoma cells. Nature. 2008;456(7222):593-8.

33. Singh SK, Hawkins C, Clarke ID, Squire JA, Bayani J, Hide T, et al. Identification of human brain tumour initiating cells. Nature. 2004;432(7015):396-401.

34. Batlle E, Clevers H. Cancer stem cells revisited. Nat Med. 2017;23(10):1124-34.

35. Chaffer CL, Brueckmann I, Scheel C, Kaestli AJ, Wiggins PA, Rodrigues LO, et al. Normal and neoplastic nonstem cells can spontaneously convert to a stem-like state. Proc Natl Acad Sci USA. 2011;108(19):7950-5.

36. Singh AK, Arya RK, Maheshwari S, Singh A, Meena S, Pandey P, et al. Tumor heterogeneity and cancer stem cell paradigm: updates in concept, controversies and clinical relevance. Int J Cancer. 2015;136(9):1991-2000.

37. Nishi M, Sakai Y, Akutsu H, Nagashima Y, Quinn G, Masui S, et al. Induction of cells with cancer stem cell properties from nontumorigenic human mammary epithelial cells by defined reprogramming factors. Oncogene. 2014;33(5):643-52.

38. Liu A, Yu X, Liu S. Pluripotency transcription factors and cancer stem cells: small genes make a big difference. Chin J Cancer. 2013;32(9):483-7.

39. Akunuru S, James Zhai Q, Zheng Y. Non-small cell lung cancer stem/progenitor cells are enriched in multiple distinct phenotypic subpopulations and exhibit plasticity. Cell Death Dis. 2012;3:e352.

40. Shao J, Fan W, Ma B, Wu Y. Breast cancer stem cells expressing different stem cell markers exhibit distinct biological characteristics. Mol Med Report. 2016;14(6):4991-8. 
41. Liu S, Cong Y, Wang D, Sun Y, Deng L, Liu Y, et al. Breast cancer stem cells transition between epithelial and mesenchymal states reflective of their normal counterparts. Stem Cell Reports. 2014;2(1):78-91.

42. Alvero AB, Chen R, Fu HH, Montagna M, Schwartz PE, Rutherford T, et al. Molecular phenotyping of human ovarian cancer stem cells unravels the mechanisms for repair and chemoresistance. Cell Cycle. 2009;8(1):158-66.

43. Ferreira JA, Peixoto A, Neves M, Gaiteiro C, Reis CA, Assaraf YG, et al. Mechanisms of cisplatin resistance and targeting of cancer stem cells: Adding glycosylation to the equation. Drug Resist Updat. 2016;24:34-54.

44. Abdullah LN, Chow EK. Mechanisms of chemoresistance in cancer stem cells. Clin Transl Med. 2013;2(1):3.

45. Levina V, Marrangoni AM, DeMarco R, Gorelik E, Lokshin AE. Drug-selected human lung cancer stem cells: cytokine network, tumorigenic and metastatic properties. PLoS One. 2008;3(8):e3077.

46. Dallas NA, Xia L, Fan F, Gray MJ, Gaur P, van Buren G, 2nd, et al. Chemoresistant colorectal cancer cells, the cancer stem cell phenotype, and increased sensitivity to insulin-like growth factor-I receptor inhibition. Cancer Res. 2009;69(5):1951-7.

47. Chen J, Li Y, Yu T-S, McKay RM, Burns DK, Kernie SG, et al. A restricted cell population propagates glioblastoma growth after chemotherapy. Nature. 2012;488(7412):522-6.

48. Jo A, Denduluri S, Zhang B, Wang Z, Yin L, Yan Z, et al. The versatile functions of Sox9 in development, stem cells, and human diseases. Genes Dis. 2014;1(2):149-61.

49. Bi W, Huang W, Whitworth DJ, Deng JM, Zhang Z, Behringer RR, et al. Haploinsufficiency of Sox9 results in defective cartilage primordia and premature skeletal mineralization. Proc Natl Acad Sci U S A. 2001;98(12):6698-703.

50. Dy $P$, Wang $W$, Bhattaram $P$, Wang $Q$, Wang L, Ballock RT, et al. Sox9 directs hypertrophic maturation and blocks osteoblast differentiation of growth plate chondrocytes. Dev Cell. 2012;22(3):597-609.

51. Furuyama K, Kawaguchi Y, Akiyama H, Horiguchi M, Kodama S, Kuhara T, et al. Continuous cell supply from a Sox9-expressing progenitor zone in adult liver, exocrine pancreas and intestine. Nat Genet. 2011;43(1):34-41.

52. Scott CE, Wynn SL, Sesay A, Cruz C, Cheung M, Gomez Gaviro MV, et al. SOX9 induces and maintains neural stem cells. Nat Neurosci. 2010;13(10):1181-9.

53. Rockich BE, Hrycaj SM, Shih HP, Nagy MS, Ferguson MA, Kopp JL, et al. Sox9 plays multiple roles in the lung epithelium during branching morphogenesis. Proc Natl Acad Sci USA. 2013;110(47):E4456-64.

54. Li L, Zhang H, Min D, Zhang R, Wu J, Qu H, et al. Sox9 activation is essential for the recovery of lung function after acute lung injury. Cell Physiol Biochem. 2015;37(3):1113-22.

55. Mertin S, McDowall SG, Harley VR. The DNA-binding specificity of SOX9 and other SOX proteins. Nucleic Acids Res. 1999;27(5):1359-64.

56. Bernard P, Tang P, Liu S, Dewing P, Harley VR, Vilain E. Dimerization of SOX9 is required for chondrogenesis, but not for sex determination. Hum Mol Genet. 2003;12(14):1755-

65.

57. Ohba S, He X, Hojo H, McMahon AP. Distinct Transcriptional Programs Underlie Sox9 Regulation of the Mammalian Chondrocyte. Cell reports. 2015;12(2):229-43.

58. Ikeda T, Kamekura S, Mabuchi A, Kou I, Seki S, Takato T, et al. The combination of SOX5, SOX6, and SOX9 (the SOX trio) provides signals sufficient for induction of permanent cartilage. Arthritis Rheum. 2004;50(11):3561-73.

59. Lefebvre V, Li P, de Crombrugghe B. A new long form of Sox5 (L-Sox5), Sox6 and Sox9 are coexpressed in chondrogenesis and cooperatively activate the type II collagen gene. EMBO J. 1998;17(19):5718-33. 
60. Huang YH, Jankowski A, Cheah KS, Prabhakar S, Jauch R. SOXE transcription factors form selective dimers on non-compact DNA motifs through multifaceted interactions between dimerization and high-mobility group domains. Sci Rep. 2015;5:10398.

61. Shi Z, Chiang Cl, Labhart P, Zhao Y, Yang J, Mistretta TA, et al. Context-specific role of SOX9 in NF-Y mediated gene regulation in colorectal cancer cells. Nucleic Acids Res. 2015;43(13):6257-69.

62. Girardot M, Bayet E, Maurin J, Fort P, Roux P, Raynaud P. SOX9 has distinct regulatory roles in alternative splicing and transcription. Nucleic Acids Res. 2018;46(17):9106-18.

63. Lefebvre V, Huang W, Harley VR, Goodfellow PN, de Crombrugghe B. SOX9 is a potent activator of the chondrocyte-specific enhancer of the pro alpha1(II) collagen gene. Mol Cell Biol. 1997;17(4):2336-46.

64. Larsimont JC, Youssef KK, Sanchez-Danes A, Sukumaran V, Defrance M, Delatte B, et al. Sox9 Controls Self-Renewal of Oncogene Targeted Cells and Links Tumor Initiation and Invasion. Cell Stem Cell. 2015;17(1):60-73.

65. Ma F, Ye H, He HH, Gerrin SJ, Chen S, Tanenbaum BA, et al. SOX9 drives WNT pathway activation in prostate cancer. J Clin Invest. 2016;126(5):1745-58.

66. Kadaja M, Keyes BE, Lin M, Pasolli HA, Genander M, Polak L, et al. SOX9: a stem cell transcriptional regulator of secreted niche signaling factors. Genes Dev. 2014;28(4):328-41.

67. Leung VYL, Gao B, Leung KKH, Melhado IG, Wynn SL, Au TYK, et al. SOX9 governs differentiation stage-specific gene expression in growth plate chondrocytes via direct concomitant transactivation and repression. PLoS Genet. 2011;7(11):e1002356.

68. Wang HY, Lian P, Zheng PS. SOX9, a potential tumor suppressor in cervical cancer, transactivates p21WAF1/CIP1 and suppresses cervical tumor growth. Oncotarget. 2015.

69. Panda DK, Miao DS, Lefebvre V, Hendy GN, Goltzman D. The transcription factor SOX9 regulates cell cycle and differentiation genes in chondrocytic CFK2 cells. J Biol Chem. $2001 ; 276(44): 41229-36$.

70. Pritchett J, Athwal V, Roberts N, Hanley NA, Hanley KP. Understanding the role of SOX9 in acquired diseases: lessons from development. Trends Mol Med. 2011;17(3):166-74.

71. Hong X, Liu W, Song R, Shah JJ, Feng X, Tsang CK, et al. SOX9 is targeted for proteasomal degradation by the E3 ligase FBW7 in response to DNA damage. Nucleic Acids Res. 2016;44(18):8855-69.

72. Suryo Rahmanto A, Savov V, Brunner A, Bolin S, Weishaupt H, Malyukova A, et al. FBW7 suppression leads to SOX9 stabilization and increased malignancy in medulloblastoma. EMBO J. 2016;35(20):2192-212.

73. Mead TJ, Wang Q, Bhattaram P, Dy P, Afelik S, Jensen J, et al. A far-upstream (-70 kb) enhancer mediates Sox9 auto-regulation in somatic tissues during development and adult regeneration. Nucleic Acids Res. 2013;41(8):4459-69.

74. Song S, Maru DM, Ajani JA, Chan C-H, Honjo S, Lin H-K, et al. Loss of TGF-beta adaptor beta2SP activates notch signaling and SOX9 expression in esophageal adenocarcinoma. Cancer Res. 2013;73(7):2159-69.

75. Capaccione KM, Hong X, Morgan KM, Liu W, Bishop JM, Liu L, et al. Sox9 mediates Notch1-induced mesenchymal features in lung adenocarcinoma. Oncotarget. 2014;5(11):363650 .

76. Bien-Willner GA, Stankiewicz P, Lupski JR. SOX9cre1, a cis-acting regulatory element located 1.1 Mb upstream of SOX9, mediates its enhancement through the SHH pathway. Hum Mol Genet. 2007;16(10):1143-56.

77. Park J, Zhang JJR, Moro A, Kushida M, Wegner M, Kim PCW. Regulation of Sox9 by Sonic Hedgehog (Shh) is essential for patterning and formation of tracheal cartilage. Developmental dynamics : an official publication of the American Association of Anatomists. 2010;239(2):514-26. 
78. Hill TP, Spater D, Taketo MM, Birchmeier W, Hartmann C. Canonical Wnt/beta-catenin signaling prevents osteoblasts from differentiating into chondrocytes. Dev Cell. 2005;8(5):72738.

79. Blache P, van de Wetering M, Duluc I, Domon C, Berta P, Freund JN, et al. SOX9 is an intestine crypt transcription factor, is regulated by the Wnt pathway, and represses the CDX2 and MUC2 genes. J Cell Biol. 2004;166(1):37-47.

80. Liu JAJ, Wu MH, Yan $\mathrm{CH}$, Chau BKH, So H, Ng A, et al. Phosphorylation of Sox9 is required for neural crest delamination and is regulated downstream of BMP and canonical Wnt signaling. Proc Natl Acad Sci USA. 2013;110(8):2882-7.

81. Coricor G, Serra R. TGF-beta regulates phosphorylation and stabilization of Sox9 protein in chondrocytes through p38 and Smad dependent mechanisms. Sci Rep. 2016;6:38616.

82. Furumatsu T, Tsuda M, Taniguchi N, Tajima $\mathrm{Y}$, Asahara $\mathrm{H}$. Smad3 induces chondrogenesis through the activation of SOX9 via CREB-binding protein/p300 recruitment. The Journal of biological chemistry. 2005;280(9):8343-50.

83. Li H, Cai H, Deng J, Tu X, Sun Y, Huang Z, et al. TGF-beta-mediated upregulation of Sox9 in fibroblast promotes renal fibrosis. Biochim Biophys Acta Mol Basis Dis. 2018;1864(2):520-32.

84. Zhou H, Qin Y, Ji S, Ling J, Fu J, Zhuang Z, et al. SOX9 activity is induced by oncogenic Kras to affect MDC1 and MCMs expression in pancreatic cancer. Oncogene. 2018;37(7):91223.

85. Sun L, Mathews LA, Cabarcas SM, Zhang X, Yang A, Zhang Y, et al. Epigenetic regulation of SOX9 by the NF-kappaB signaling pathway in pancreatic cancer stem cells. Stem Cells. 2013;31(8):1454-66.

86. Song S, Ajani JA, Honjo S, Maru DM, Chen Q, Scott AW, et al. Hippo coactivator YAP1 upregulates SOX9 and endows esophageal cancer cells with stem-like properties. Cancer Res. 2014;74(15):4170-82.

87. Murakami S, Kan M, McKeehan WL, de Crombrugghe B. Up-regulation of the chondrogenic Sox9 gene by fibroblast growth factors is mediated by the mitogen-activated protein kinase pathway. Proc Natl Acad Sci USA. 2000;97(3):1113-8.

88. Huang W, Zhou X, Lefebvre V, de Crombrugghe B. Phosphorylation of SOX9 by cyclic AMP-dependent protein kinase A enhances SOX9's ability to transactivate a Col2a1 chondrocyte-specific enhancer. Mol Cell Biol. 2000;20(11):4149-58.

89. Matheu A, Collado M, Wise C, Manterola L, Cekaite L, Tye AJ, et al. Oncogenicity of the developmental transcription factor Sox9. Cancer Res. 2012;72(5):1301-15.

90. Ruan H, Hu S, Zhang H, Du G, Li X, Li X, et al. Upregulated SOX9 expression indicates worse prognosis in solid tumors: a systematic review and meta-analysis. Oncotarget. 2017;8(68):113163-73.

91. Lei B, Zhang YX, Liu T, Li YW, Pang D. Sox9 upregulation in breast cancer is correlated with poor prognosis and the CD44(+)/CD24(-/low) phenotype. Int J Clin Exp Pathol. 2016;9(7):7345-51.

92. Cheng PF, Shakhova O, Widmer DS, Eichhoff OM, Zingg D, Frommel SC, et al. Methylation-dependent SOX9 expression mediates invasion in human melanoma cells and is a negative prognostic factor in advanced melanoma. Genome Biol. 2015;16.

93. Shakhova O, Cheng P, Mishra PJ, Zingg D, Schaefer SM, Debbache J, et al. Antagonistic Cross-Regulation between Sox9 and Sox10 Controls an Anti-tumorigenic Program in Melanoma. PLoS Genet. 2015;11(1).

94. Yang X, Liang R, Liu C, Liu JA, Cheung MPL, Liu X, et al. SOX9 is a dose-dependent metastatic fate determinant in melanoma. Journal of experimental \& clinical cancer research : CR. 2019;38(1):17. 
95. Prevostel C, Blache P. The dose-dependent effect of SOX9 and its incidence in colorectal cancer. European journal of cancer (Oxford, England : 1990). 2017;86:150-7.

96. Marcker Espersen ML, Linnemann D, Christensen IJ, Alamili M, Troelsen JT, Hogdall E. SOX9 expression predicts relapse of stage II colon cancer patients. Hum Pathol. 2016;52:3846.

97. Prevostel C, Rammah-Bouazza C, Trauchessec H, Canterel-Thouennon L, Busson M, Ychou M, et al. SOX9 is an atypical intestinal tumor suppressor controlling the oncogenic Wnt/SS-catenin signaling. Oncotarget. 2016;7(50):82228-43.

98. Carrasco-Garcia E, Lopez L, Aldaz P, Arevalo S, Aldaregia J, Egana L, et al. SOX9regulated cell plasticity in colorectal metastasis is attenuated by rapamycin. Sci Rep. 2016;6:32350.

99. Lu B, Fang Y, Xu J, Wang L, Xu F, Xu E, et al. Analysis of SOX9 expression in colorectal cancer. Am J Clin Pathol. 2008;130(6):897-904.

100. Kopp JL, von Figura G, Mayes E, Liu FF, Dubois CL, Morris JPt, et al. Identification of Sox9-dependent acinar-to-ductal reprogramming as the principal mechanism for initiation of pancreatic ductal adenocarcinoma. Cancer Cell. 2012;22(6):737-50.

101. Cui Y, Fang W, Li C, Tang K-J, Zhang J, Lei Y, et al. Development and validation of novel signature to predict overall survival in "driver-gene-negative" lung adenocarcinoma (LUAD): results of a multicenter study. Clinical cancer research : an official journal of the American Association for Cancer Research. 2018.

102. Liu C, Liu L, Chen X, Cheng J, Zhang H, Shen J, et al. Sox9 Regulates Self-Renewal and Tumorigenicity by Promoting Symmetrical Cell Division of Cancer Stem Cells in Hepatocellular Carcinoma. Hepatology. 2016.

103. Guo W, Keckesova Z, Donaher JL, Shibue T, Tischler V, Reinhardt F, et al. Slug and Sox9 cooperatively determine the mammary stem cell state. Cell. 2012;148(5):1015-28.

104. Luanpitpong S, Li J, Manke A, Brundage K, Ellis E, McLaughlin SL, et al. SLUG is required for SOX9 stabilization and functions to promote cancer stem cells and metastasis in human lung carcinoma. Oncogene. 2016;35(22):2824-33.

105. Santos JC, Carrasco-Garcia E, Garcia-Puga M, Aldaz P, Montes M, Fernandez-Reyes $\mathrm{M}$, et al. SOX9 Elevation Acts with Canonical WNT Signaling to Drive Gastric Cancer Progression. Cancer Res. 2016.

106. Candy PA, Phillips MR, Redfern AD, Colley SM, Davidson JA, Stuart LM, et al. Notchinduced transcription factors are predictive of survival and 5-fluorouracil response in colorectal cancer patients. Br J Cancer. 2013;109(4):1023-30.

107. Riemenschnitter C, Teleki I, Tischler V, Guo W, Varga Z. Stability and Prognostic Value of Slug, Sox9 and Sox10 Expression in Breast Cancers Treated with Neoadjuvant Chemotherapy. Mod Pathol. 2013;26:65a-a.

108. Marchitti SA, Brocker C, Stagos D, Vasiliou V. Non-P450 aldehyde oxidizing enzymes: the aldehyde dehydrogenase superfamily. Expert Opin Drug Metab Toxicol. 2008;4(6):697-720.

109. Tomita H, Tanaka K, Tanaka T, Hara A. Aldehyde dehydrogenase $1 \mathrm{~A} 1$ in stem cells and cancer. Oncotarget. 2016;7(10):11018-32.

110. Rodriguez-Torres M, Allan AL. Aldehyde dehydrogenase as a marker and functional mediator of metastasis in solid tumors. Clin Exp Metastasis. 2016;33(1):97-113.

111. Storms RW, Trujillo AP, Springer JB, Shah L, Colvin OM, Ludeman SM, et al. Isolation of primitive human hematopoietic progenitors on the basis of aldehyde dehydrogenase activity. Proc Natl Acad Sci U S A. 1999;96(16):9118-23.

112. Marcato P, Dean CA, Giacomantonio CA, Lee PW. Aldehyde dehydrogenase: its role as a cancer stem cell marker comes down to the specific isoform. Cell Cycle. $2011 ; 10(9): 1378-84$.

113. Zhou L, Sheng D, Wang D, Ma W, Deng Q, Deng L, et al. Identification of cancer-type specific expression patterns for active aldehyde dehydrogenase (ALDH) isoforms in ALDEFLUOR assay. Cell Biol Toxicol. 2018. 
114. Pors K, Moreb JS. Aldehyde dehydrogenases in cancer: an opportunity for biomarker and drug development? Drug Discov Today. 2014;19(12):1953-63.

115. van den Hoogen C, van der Horst G, Cheung H, Buijs JT, Lippitt JM, Guzman-Ramirez $\mathrm{N}$, et al. High aldehyde dehydrogenase activity identifies tumor-initiating and metastasisinitiating cells in human prostate cancer. Cancer Res. 2010;70(12):5163-73.

116. Zhao W, Zang C, Zhang T, Li J, Liu R, Feng F, et al. Clinicopathological characteristics and prognostic value of the cancer stem cell marker ALDH1 in ovarian cancer: a meta-analysis. Onco Targets Ther. 2018;11:1821-31.

117. Huo W, Du M, Pan X, Zhu X, Li Z. Prognostic value of ALDH1 expression in lung cancer: a meta-analysis. Int J Clin Exp Med. 2015;8(2):2045-51.

118. Wei D, Peng J-J, Gao H, Zhang T, Tan Y, Hu Y-H. ALDH1 Expression and the Prognosis of Lung Cancer: A Systematic Review and Meta-Analysis. Heart Lung Circ. $2015 ; 24(8): 780-8$.

119. Chen J, Xia Q, Jiang B, Chang W, Yuan W, Ma Z, et al. Prognostic Value of Cancer Stem Cell Marker ALDH1 Expression in Colorectal Cancer: A Systematic Review and MetaAnalysis. PLoS One. 2015;10(12):e0145164.

120. Zhou C, Sun B. The prognostic role of the cancer stem cell marker aldehyde dehydrogenase 1 in head and neck squamous cell carcinomas: a meta-analysis. Oral Oncol. 2014;50(12):1144-8.

121. Januchowski R, Wojtowicz K, Zabel M. The role of aldehyde dehydrogenase (ALDH) in cancer drug resistance. Biomed Pharmacother. 2013;67(7):669-80.

122. Tanei T, Morimoto K, Shimazu K, Kim SJ, Tanji Y, Taguchi T, et al. Association of breast cancer stem cells identified by aldehyde dehydrogenase 1 expression with resistance to sequential Paclitaxel and epirubicin-based chemotherapy for breast cancers. Clinical cancer research : an official journal of the American Association for Cancer Research. 2009;15(12):4234-41.

123. Li J, Zhang B, Yang YF, Jin J, Liu YH. Aldehyde dehydrogenase 1 as a predictor of the neoadjuvant chemotherapy response in breast cancer: A meta-analysis. Medicine. 2018;97(34):e12056.

124. Ajani JA, Wang X, Song S, Suzuki A, Taketa T, Sudo K, et al. ALDH-1 expression levels predict response or resistance to preoperative chemoradiation in resectable esophageal cancer patients. Mol Oncol. 2014;8(1):142-9.

125. Januchowski R, Wojtowicz K, Sterzyska K, Sosiska P, Andrzejewska M, Zawierucha P, et al. Inhibition of ALDH1A1 activity decreases expression of drug transporters and reduces chemotherapy resistance in ovarian cancer cell lines. Int J Biochem Cell Biol. 2016;78:248-59.

126. Duong HQ, Hwang JS, Kim HJ, Kang HJ, Seong YS, Bae I. Aldehyde dehydrogenase $1 \mathrm{~A} 1$ confers intrinsic and acquired resistance to gemcitabine in human pancreatic adenocarcinoma MIA PaCa-2 cells. Int J Oncol. 2012;41(3):855-61.

127. Barr MP, Gray SG, Hoffmann AC, Hilger RA, Thomale J, O'Flaherty JD, et al. Generation and characterisation of cisplatin-resistant non-small cell lung cancer cell lines displaying a stem-like signature. PLoS One. 2013;8(1):e54193.

128. Ginestier C, Wicinski J, Cervera N, Monville F, Finetti P, Bertucci F, et al. Retinoid signaling regulates breast cancer stem cell differentiation. Cell Cycle. 2009;8(20):3297-302.

129. Ying M, Wang S, Sang Y, Sun P, Lal B, Goodwin CR, et al. Regulation of glioblastoma stem cells by retinoic acid: role for Notch pathway inhibition. Oncogene. 2011;30(31):3454-67.

130. Degos L, Wang ZY. All trans retinoic acid in acute promyelocytic leukemia. Oncogene. $2001 ; 20(49): 7140-5$.

131. Koppaka V, Thompson DC, Chen Y, Ellermann M, Nicolaou KC, Juvonen RO, et al. Aldehyde dehydrogenase inhibitors: a comprehensive review of the pharmacology, mechanism of action, substrate specificity, and clinical application. Pharmacol Rev. 2012;64(3):520-39. 
132. Vassalli G. Aldehyde Dehydrogenases: Not Just Markers, but Functional Regulators of Stem Cells. Stem Cells Int. 2019;2019:3904645.

133. von Eitzen U, Meier-Tackmann D, Agarwal DP, Goedde HW. Detoxification of cyclophosphamide by human aldehyde dehydrogenase isozymes. Cancer Lett. 1994;76(1):459.

134. Moreb J, Zucali JR, Zhang Y, Colvin MO, Gross MA. Role of aldehyde dehydrogenase in the protection of hematopoietic progenitor cells from 4-hydroperoxycyclophosphamide by interleukin 1 beta and tumor necrosis factor. Cancer Res. 1992;52(7):1770-4.

135. Mizuno T, Suzuki N, Makino H, Furui T, Morii E, Aoki H, et al. Cancer stem-like cells of ovarian clear cell carcinoma are enriched in the ALDH-high population associated with an accelerated scavenging system in reactive oxygen species. Gynecol Oncol. 2015;137(2):299305.

136. Raha D, Wilson TR, Peng J, Peterson D, Yue P, Evangelista M, et al. The cancer stem cell marker aldehyde dehydrogenase is required to maintain a drug-tolerant tumor cell subpopulation. Cancer Res. 2014;74(13):3579-90.

137. Kang JH, Lee S-H, Hong D, Lee J-S, Ahn H-S, Ahn J-H, et al. Aldehyde dehydrogenase is used by cancer cells for energy metabolism. Exp Mol Med. 2016;48(11):e272.

138. Kim D, Choi BH, Ryoo IG, Kwak MK. High NRF2 level mediates cancer stem cell-like properties of aldehyde dehydrogenase (ALDH)-high ovarian cancer cells: inhibitory role of alltrans retinoic acid in ALDH/NRF2 signaling. Cell Death Dis. 2018;9(9):896.

139. Patel M, Lu L, Zander DS, Sreerama L, Coco D, Moreb JS. ALDH1A1 and ALDH3A1 expression in lung cancers: correlation with histologic type and potential precursors. Lung Cancer. 2008;59(3):340-9.

140. Shao C, Sullivan JP, Girard L, Augustyn A, Yenerall P, Rodriguez-Canales J, et al. Essential role of aldehyde dehydrogenase 1A3 for the maintenance of non-small cell lung cancer stem cells is associated with the STAT3 pathway. Clin Cancer Res. 2014;20(15):415466.

141. Marcato P, Dean CA, Pan D, Araslanova R, Gillis M, Joshi M, et al. Aldehyde dehydrogenase activity of breast cancer stem cells is primarily due to isoform ALDH1A3 and its expression is predictive of metastasis. Stem cells (Dayton, Ohio). 2011;29(1):32-45.

142. Croker AK, Rodriguez-Torres M, Xia Y, Pardhan S, Leong HS, Lewis JD, et al. Differential Functional Roles of ALDH1A1 and ALDH1A3 in Mediating Metastatic Behavior and Therapy Resistance of Human Breast Cancer Cells. Int J Mol Sci. 2017;18(10).

143. Ribes V, Wang Z, Dolle P, Niederreither K. Retinaldehyde dehydrogenase 2 (RALDH2)mediated retinoic acid synthesis regulates early mouse embryonic forebrain development by controlling FGF and sonic hedgehog signaling. Development (Cambridge, England). 2006;133(2):351-61.

144. Kawasoe M, Yamamoto Y, Okawa K, Funato T, Takeda M, Hara T, et al. Acquired resistance of leukemic cells to AraC is associated with the upregulation of aldehyde dehydrogenase 1 family member A2. Exp Hematol. 2013;41(7):597-603.e2.

145. Moreb JS, Ucar D, Han S, Amory JK, Goldstein AS, Ostmark B, et al. The enzymatic activity of human aldehyde dehydrogenases $1 \mathrm{~A} 2$ and 2 (ALDH1A2 and ALDH2) is detected by Aldefluor, inhibited by diethylaminobenzaldehyde and has significant effects on cell proliferation and drug resistance. Chem Biol Interact. 2012;195(1):52-60.

146. Graham CE, Brocklehurst K, Pickersgill RW, Warren MJ. Characterization of retinaldehyde dehydrogenase 3. The Biochemical journal. 2006;394(Pt 1):67-75.

147. Canino C, Luo Y, Marcato P, Blandino G, Pass HI, Cioce M. A STAT3NFkB/DDIT3/CEBPbeta axis modulates ALDH1A3 expression in chemoresistant cell subpopulations. Oncotarget. 2015;6(14):12637-53. 
148. Mao P, Joshi K, Li J, Kim S-H, Li P, Santana-Santos L, et al. Mesenchymal glioma stem cells are maintained by activated glycolytic metabolism involving aldehyde dehydrogenase $1 \mathrm{~A} 3$. Proc Natl Acad Sci USA. 2013;110(21):8644-9.

149. Flahaut M, Jauquier N, Chevalier N, Nardou K, Balmas Bourloud K, Joseph J-M, et al. Aldehyde dehydrogenase activity plays a Key role in the aggressive phenotype of neuroblastoma. BMC Cancer. 2016;16(1):781.

150. Perez-Alea M, McGrail K, Sanchez-Redondo S, Ferrer B, Fournet G, Cortes J, et al. ALDH1A3 is epigenetically regulated during melanocyte transformation and is a target for melanoma treatment. Oncogene. 2017;36(41):5695-708.

151. Singh S, Arcaroli J, Chen Y, Thompson DC, Messersmith W, Jimeno A, et al. ALDH1B1 Is Crucial for Colon Tumorigenesis by Modulating Wnt/beta-Catenin, Notch and PI3K/Akt Signaling Pathways. PLoS One. 2015;10(5):e0121648.

152. Krupenko SA, Oleinik NV. 10-formyltetrahydrofolate dehydrogenase, one of the major folate enzymes, is down-regulated in tumor tissues and possesses suppressor effects on cancer cells. Cell growth \& differentiation : the molecular biology journal of the American Association for Cancer Research. 2002;13(5):227-36.

153. Piskounova E, Agathocleous M, Murphy MM, Hu Z, Huddlestun SE, Zhao Z, et al. Oxidative stress inhibits distant metastasis by human melanoma cells. Nature. 2015;527(7577):186-91.

154. Moreb JS, Mohuczy D, Muhoczy D, Ostmark B, Zucali JR. RNAi-mediated knockdown of aldehyde dehydrogenase class-1A1 and class-3A1 is specific and reveals that each contributes equally to the resistance against 4-hydroperoxycyclophosphamide. Cancer Chemother Pharmacol. 2007;59(1):127-36.

155. Parajuli B, Fishel ML, Hurley TD. Selective ALDH3A1 inhibition by benzimidazole analogues increase mafosfamide sensitivity in cancer cells. J Med Chem. 2014;57(2):449-61.

156. Saw Y-T, Yang J, Ng S-K, Liu S, Singh S, Singh M, et al. Characterization of aldehyde dehydrogenase isozymes in ovarian cancer tissues and sphere cultures. BMC Cancer. 2012;12:329.

157. Marchitti SA, Brocker C, Orlicky DJ, Vasiliou V. Molecular characterization, expression analysis, and role of ALDH3B1 in the cellular protection against oxidative stress. Free Radic Biol Med. 2010;49(9):1432-43.

158. Yoon K-A, Nakamura Y, Arakawa H. Identification of ALDH4 as a p53-inducible gene and its protective role in cellular stresses. J Hum Genet. 2004;49(3):134-40.

159. Kaur H, Mao S, Li Q, Sameni M, Krawetz SA, Sloane BF, et al. RNA-Seq of human breast ductal carcinoma in situ models reveals aldehyde dehydrogenase isoform $5 \mathrm{~A} 1$ as a novel potential target. PLoS One. 2012;7(12):e50249.

160. Rexer BN, Zheng WL, Ong DE. Retinoic acid biosynthesis by normal human breast epithelium is via aldehyde dehydrogenase 6 , absent in MCF-7 cells. Cancer Res. $2001 ; 61(19): 7065-70$.

161. van den Hoogen C, van der Horst G, Cheung H, Buijs JT, Pelger RCM, van der Pluijm $G$. The aldehyde dehydrogenase enzyme $7 A 1$ is functionally involved in prostate cancer bone metastasis. Clin Exp Metastasis. 2011;28(7):615-25.

162. Grinde MT, Hilmarsdottir B, Tunset HM, Henriksen IM, Kim J, Haugen MH, et al. Glutamine to proline conversion is associated with response to glutaminase inhibition in breast cancer. Breast cancer research : BCR. 2019;21(1):61.

163. Alam M, Ahmad R, Rajabi H, Kharbanda A, Kufe D. MUC1-C oncoprotein activates ERK-->C/EBPbeta signaling and induction of aldehyde dehydrogenase 1A1 in breast cancer cells. J Biol Chem. 2013;288(43):30892-903.

164. Cojoc M, Peitzsch C, Kurth I, Trautmann F, Kunz-Schughart LA, Telegeev GD, et al. Aldehyde Dehydrogenase Is Regulated by beta-Catenin/TCF and Promotes Radioresistance in Prostate Cancer Progenitor Cells. Cancer Res. 2015;75(7):1482-94. 
165. Elizondo G, Corchero J, Sterneck E, Gonzalez FJ. Feedback inhibition of the retinaldehyde dehydrogenase gene $A L D H 1$ by retinoic acid through retinoic acid receptor alpha and CCAAT/enhancer-binding protein beta. The Journal of biological chemistry. 2000;275(50):39747-53.

166. Hoshino Y, Nishida J, Katsuno Y, Koinuma D, Aoki T, Kokudo N, et al. Smad4 Decreases the Population of Pancreatic Cancer-Initiating Cells through Transcriptional Repression of ALDH1A1. The American journal of pathology. 2015;185(5):1457-70.

167. Shuang ZY, Wu WC, Xu J, Lin G, Liu YC, Lao XM, et al. Transforming growth factorbeta1-induced epithelial-mesenchymal transition generates ALDH-positive cells with stem cell properties in cholangiocarcinoma. Cancer Lett. 2014;354(2):320-8.

168. Bhola NE, Balko JM, Dugger TC, Kuba MG, Sanchez V, Sanders M, et al. TGF-beta inhibition enhances chemotherapy action against triple-negative breast cancer. $\mathrm{J}$ Clin Invest. 2013;123(3):1348-58.

169. Nishida J, Miyazono K, Ehata S. Decreased TGFBR3/betaglycan expression enhances the metastatic abilities of renal cell carcinoma cells through TGF-beta-dependent and independent mechanisms. Oncogene. 2018;37(16):2197-212.

170. Zhao D, Mo Y, Li M-T, Zou S-W, Cheng Z-L, Sun Y-P, et al. NOTCH-induced aldehyde dehydrogenase $1 \mathrm{~A} 1$ deacetylation promotes breast cancer stem cells. The Journal of clinical investigation. 2014;124(12):5453-65.

171. Wang J, Nikhil K, Viccaro K, Chang L, White J, Shah K. Phosphorylation-dependent regulation of ALDH1A1 by Aurora kinase $A$ : insights on their synergistic relationship in pancreatic cancer. BMC Biol. 2017;15(1):10. 


\section{Chapter 2: SOX9-ALDH axis determines resistance to chemotherapy in non-small cell lung cancer}

Maria A. Voronkova ${ }^{1}$, Liying W. Rojanasakul ${ }^{2}$, Chayanin Kiratipaiboon ${ }^{3}$ and Yon Rojanasakul ${ }^{1,3^{*}}$

${ }^{1}$ West Virginia University Cancer Institute, ${ }^{3}$ Department of Pharmaceutical Sciences, West Virginia University, Morgantown, WV 26506, United States; ${ }^{2}$ Allergy and Clinical Immunology Branch, National Institute for Occupational Safety and Health, Morgantown, WV 26505, United States.

Running title: SOX9 promotes chemoresistance through ALDH

Keywords: cancer stem-like cells, chemoresistance, SOX9, ALDH1A1, non-small cell lung cancer 


\begin{abstract}
Chemotherapy resistance and tumor relapse are the major contributors to low patient survival, and both have been largely attributed to cancer stem-like cells (CSCs) or tumor initiating cells (TICs). Moreover, most conventional therapies are not effective against CSCs, which necessitates the discovery of CSC-specific biomarkers and drug targets. Here, we demonstrated that the embryonic transcription factor SOX9 is an important regulator of acquired chemoresistance in non-small cell lung cancer (NSCLC). Our results showed that SOX9 expression is elevated in NSCLC cells after treatment with the chemotherapeutic cisplatin and that overexpression of SOX9 correlated with worse overall survival in lung cancer patients. We further demonstrated that SOX9 knockdown increases cellular sensitivity to cisplatin, whereas its overexpression promotes drug resistance. Moreover, this transcription factor promotes stem-like properties of NSCLC cells and increases their ALDH activity, which was identified as the key mechanism of SOX9-induced chemoresistance. Finally, we showed that ALDH1A1 is a direct transcriptional target of SOX9 based on chromatin immunoprecipitation and luciferase reporter assays. Taken together, our novel findings on the role of SOX9-ALDH axis support the use of this CSC regulator as a prognostic marker of cancer chemoresistance and as a potential drug target for CSC therapy.
\end{abstract}




\section{Introduction}

SOX9 is a member of the high-mobility group (HMG)-box class of transcription factors and controls critical stages in embryonic development, stem cell homeostasis and differentiation. Germline SOX9 mutations cause severe cartilage and bone malformations, and male sex reversal (1). In adults, SOX9 is expressed in stem cells in multiple tissues, including central nervous system, skin, intestine, liver and pancreas (13). In lungs, SOX9 regulates branching morphogenesis during embryonic development and a response to injuries in adults $(4,5)$. SOX9 has also been implicated in the formation and progression of multiple cancer types (6). It is overexpressed in various solid tumors, such as breast, prostate, pancreatic and lung cancers. Furthermore, SOX9 overexpression positively correlates with poor overall and disease-free survival in these malignancies $(7,8)$, which could be due to its effect on tumor initiating cells or cancer stem cells (CSCs).

CSCs are a small tumor cell population that is highly tumorigenic and generally resistant to apoptosis, chemo- and radiation therapy, thus thought to be a major source of drug resistance and recurrence in cancer (9). As imbalance of stem cell homeostasis and differentiation may contribute to tumorigenesis, many stem cell transcription factors, including SOX9, have been linked to CSC regulation. Indeed, SOX9 has been shown to promote self-renewal and inhibit cell differentiation in basal cell carcinoma (10). This transcription factor is also highly expressed in liver CSCs and controls self-renewal and symmetrical cell division, promoting tumorigenicity in hepatocellular carcinoma (11). In

lungs, a recent study by our group showed that SOX9 expression is associated with stemness of lung carcinoma as indicated by their side population and sphere formation properties (12), although the underlying mechanisms are not known.

Consistent with its role in CSC regulation, increasing evidence suggests that SOX9 may play a role in chemotherapy resistance (13-16). However, a detailed understanding of the process remains to be elucidated. We hypothesized that SOX9 plays a role in lung cancer progression and chemoresistance by inducing cancer stemlike properties. We show here that SOX9 is upregulated upon exposure to cisplatin and 
positively regulates cancer stem-like properties and chemoresistance of NSCLC cells. Moreover, we demonstrated for the first time that the stem-cell marker ALDH1A1 is a direct transcriptional target of SOX9 that drives chemotherapy response in NSCLC cells. 


\section{Results}

High SOX9 expression correlates with poor survival in NSCLC patients and is induced by cisplatin exposure in human lung cancer cells

SOX9 is reported to be overexpressed in a number of cancer types, including lung cancer. We analyzed The Cancer Genome Atlas (TCGA) NSCLC patient cohort to determine whether SOX9 overexpression could predict patient survival. Indeed, higher SOX9 expression correlates with poor overall survival in adenocarcinoma and squamous cell carcinoma, two major histological types of NSCLC (Figure 1A, B). SOX9 is overexpressed in tumors compared to normal tissues in patient samples $(17,18)$. Consistent with these data, we found that SOX9 expression is highly upregulated in most NSCLC cell lines as compared to primary lung epithelial cells and several nontumorigenic lung epithelial cell lines (Figure 1C). Together, these observations support the role of SOX9 in lung cancer progression.

Multiple studies have reported an increased expression of several stem cell markers in drug resistant cells (19-21). We tested whether SOX9 expression is upregulated in lung cancer cells in response to cisplatin treatment. NSCLC cells H460, A549 and $\mathrm{H} 1299$ were treated with cisplatin at the $\mathrm{IC}_{50}$ concentrations and analyzed for SOX9 expression by RT-qPCR and Western blotting. Figures $1 \mathrm{D}$ and $1 \mathrm{~F}$ show that SOX9 protein and mRNA levels were highly elevated following a 2-3-day exposure to the drug. Furthermore, these cells become highly resistant to cisplatin upon subsequent exposures (Figure 1E, Suppl. Fig. 1A), suggesting a potential role of SOX9 in resistance to cisplatin.

\section{SOX9 overexpression promotes chemoresistance in NSCLC cells}

Next, we examined the effects of SOX9 overexpression and knockdown on cell survival upon treatment with chemotherapeutic drugs. Our results showed that SOX9 knockdown in two NSCLC cell lines, H460 and A549 (see Suppl. Fig. 1 for knockdown and overexpression levels), increased drug sensitivity (Figure 2A, Suppl. Fig. 1B, C), whereas SOX9 overexpression reduced the sensitivity of these cells (Figure 2B, C). Treatment with other chemo drugs, such as paclitaxel and etoposide, yielded similar 
results (Suppl. Fig. 1D, E), indicating a broad role of SOX9 in cell survival upon drug exposure. While the standard cell viability assay provides a valuable insight into an acute cellular response to drugs, evaluating a more prolonged effect of drug exposure is more clinically relevant with regards to the development of resistance. Thus, colony formation assay is often used as a surrogate for long-term cell survival after drug treatment. In this study, we pre-exposed the cells to cisplatin for 2 days, let them recover for 4 days in drug-free media, and then the cells were plated at a very low density for colony formation. The number of colonies formed by the cisplatin-treated cells was normalized to that of untreated cells to account for any initial differences in colony formation caused by SOX9 knockdown. Our results showed that SOX9 knockdown significantly reduced the number of colonies as compared to vector control (Figure 2D), while SOX9 overexpression promoted the colony formation (Figure 2E). These observations are consistent with the cell viability results and indicate a positive regulatory role of SOX9 in drug resistance of NSCLC cells.

\section{SOX9 positively regulates cancer stem-like properties of NSCLC cells}

A growing body of evidence suggests that a particular cancer cell population, namely CSCs, may be responsible for therapeutic resistance and tumor relapses (22). We therefore investigated the role of SOX9 as a CSC regulator in NSCLC cells. First, tumor sphere formation was performed in SOX9 knockdown and overexpressing cells. Sphere formation assay is widely used to evaluate multipotency and self-renewal of stem cells in normal and cancerous tissues and serves as a measure for CSC frequency in vitro $(23,24)$. We found that the tumor sphere formation was substantially reduced in SOX9 knockdown cells (Figure 3A); whereas it was enhanced in overexpression experiments (Figure 3B). This effect was also retained during the formation of secondary spheres (Figure $3 \mathrm{C}$ ), confirming that SOX9 positively regulates self-renewal properties of NSCLC cells. In line with these results, pluripotencyassociated transcription factors Oct3/4, Nanog, SOX2 and KLF4 (25) were suppressed in SOX9 knockdown cells (Figure 3D, Suppl. Fig. 3B). Importantly, SOX9 confers cisplatin resistance under stem cell-selective conditions during sphere formation (Figure 3E, Suppl. Fig. 2 for A549 cells), suggesting that SOX9 regulates chemoresistance of 
CSCs. This result cannot be explained by the initial difference in cell proliferation, since tumor sphere formation in the presence of the drug was normalized to that of untreated cells. In addition, SOX9 overexpressing cells grew slower than the control cells (Suppl. Fig. 2C). Collectively, these observations indicate that high SOX9 expression is associated with stem-like properties of NSCLC cells.

\section{ALDH1A1 is a downstream target of SOX9 and regulates chemoresistance}

In search for the underlying mechanisms of CSC regulation by SOX9, we observed a dramatic decrease in ALDH1A1 expression in SOX9 knockdown cells (Figure 4A). The aldehyde dehydrogenase isoform ALDH1A1 belongs to a group of aldehyde oxidizing enzymes that includes 19 members in humans (26). While all group members participate in detoxification of endogenous and exogenous products of metabolism, ALDH1A1 is particularly known as a universal CSC marker (27). Furthermore, ALDH1A1 was shown to regulate cell proliferation and cancer drug resistance (28-30). Thus, we evaluated ALDH1A1 as a potential downstream effector of SOX9. Consistent with the knockdown experiments, SOX9 overexpression upregulated ALDH1A1 at the protein (Figure 4B) and mRNA (Figure 4C) levels. Moreover, ALDH enzymatic activity, measured by the flow cytometry-based Aldefluor ${ }^{\circledR}$ assay, was also elevated upon SOX9 overexpression (Figure 4D). We also profiled mRNA levels of all 19 human ALDH isoforms in SOX9 knockdown cells since the Aldefluor ${ }^{\circledR}$ assay appears to be not isoform-specific $(31,32)$. Our results showed that only ALDH1A1 expression was suppressed by SOX9 knockdown (Figure 4E), further suggesting that ALDH1A1 acts downstream of SOX9.

We next evaluated the role of SOX9-mediated ALDH1A1 upregulation in drug resistance. We observed that ALDH1A1 expression is elevated during cisplatin treatment (Figure $5 \mathrm{~A}$ ) and that inhibition of ALDH activity by a known ALDH blocker N, $\mathrm{N}$-diethylaminobenzaldehyde (DEAB) reduced cell viability upon exposure to the drug (Figure 5B, Suppl. Fig 3A). Furthermore, the ALDH inhibitor reversed the effect of SOX9 overexpression on cisplatin resistance (Figure $5 \mathrm{C}$ ), suggesting that SOX9 mediates its chemoresistance effect through ALDH1A1. 


\section{ALDH1A1 is regulated by SOX9 at the transcriptional level}

Next, we investigated the underlying mechanism of ALDH1A1 regulation by SOX9. A time-course study of ALDH1A1 expression in cells with doxycycline-inducible SOX9 overexpression was conducted first. Interestingly, ALDH1A1 expression was upregulated as early as 4 hours after doxycycline was added (Figure 6A), suggesting that SOX9 may induce ALDH1A1 expression at the transcription level. We next performed chromatin immunoprecipitation (ChIP) followed by qPCR analysis. Validated primers for a known SOX9 target COL2A1 (33) were used as a positive control for SOX9 antibody while several primer sets were designed for the ALDH1A1 promoter (Figure 6B). In support of the previous results, SOX9 binding was enriched at several promoter locations, but not in intron 1, 4.5kb downstream of the transcription start site (Figure 6C). To further assess whether SOX9 could directly activate transcription from the ALDH1A1 promoter, we performed luciferase reporter assay. Cells were transfected with an empty luciferase reporter plasmid or a reporter plasmid containing $1 \mathrm{~kb}$ long ALDH1A1 promoter (Figure 6B). A substantial increase in luciferase signal was observed in SOX9 overexpressing cells in comparison to vector control cells (Figure 6D). A similar result was observed in HEK293 cells transfected with both SOX9 and luciferase reporter plasmids (Suppl. Fig 3C). Collectively, our observations indicate that ALDH1A1 is a direct transcriptional target of SOX9. 


\section{Discussion}

Non-small cell lung cancer (NSCLC) is the second most common cancer in both men and women and a leading cause of cancer-related death worldwide. It claims around 150,000 lives yearly in the U.S. alone (34). Despite recent advances in cancer therapy, chemotherapy remains a vital component of the overall treatment strategies for lung cancer. However, patient survival continues to be poor due to intrinsic and newly acquired drug resistance, which is an unresolved clinical problem. We identified SOX9 as a potential regulator of cancer cell survival during chemotherapy based on its ability to regulate chemoresistant CSCs. We also observed a dramatic increase in SOX9 levels in cisplatin-treated cells. In contrast, Hong et al. (14) reported that SOX9 was degraded by cisplatin and other DNA damaging agents such as doxorubicin. This apparent contradiction may be explained by the fact that the latter study used extremely high drug concentrations (e.g. $30 \mu \mathrm{M}$ for cisplatin and 7.4 $\mu \mathrm{M}$ for doxorubicin) and a short exposure time that could result in different cellular responses from those observed in this study. In another report, SOX9 expression was found to be upregulated in gastric cell lines after a low-dose $(10 \mu \mathrm{M})$ long-term (5-day) exposure to cisplatin (13). This result is consistent with our findings in lung cancer cells and suggests that SOX9 upregulation may be important for cancer cell survival under a selective therapeutic pressure. This notion is supported by clinical data showing a poor survival outcome in patients with high SOX9 expression (Figure 1A; (7))

Our major objective was to uncover a mechanism by which SOX9 mediates its effect on chemoresistance. We showed that ALDH1A1 (also identified as ALDH1), which is a common biomarker for normal and cancer stem cells, is a direct transcriptional target of SOX9. According to the cancer stem cell theory, tumors contain a small population of cells that is highly tumorigenic, drug- and apoptosis resistant and possesses stem-like properties such as the ability to self-renew and differentiate (9). The human ALDH family of proteins includes 19 members but ALDH1A1 is the main isozyme used to identify and/or isolate normal and cancer stem cells in multiple tissues (30). ALDH1A1 is overexpressed in many cancer types and usually correlates with poor prognosis. For example, high ALDH1 expression correlates with poor overall survival in 
ovarian cancer (35). Moreover, elevated ALDH1 expression after neoadjuvant chemotherapy is associated with poor response and early relapse in ovarian, breast and esophageal cancers $(36,37)$. While individual reports do not always agree on the prognostic value of ALDH1A1 overexpression, meta-analysis studies demonstrated a strong correlation between ALDH1 expression and overall and disease-free survival in lung cancer $(38,39)$, colorectal cancer (40) and head and neck squamous cell carcinomas (41). Other studies showed that ALDH1A1 is overexpressed in drugresistant cell lines $(19,29,42)$. Furthermore, high ALDH activity, typical for stem cells, could support CSC survival. For instance, ALDH1A1 can detoxify an active derivate of cyclophosphamide, 4-hydroxycyclophosphamide, and therefore directly protect cancer cells from drugs $(43,44)$. A recent study also reported that $A L D H^{\text {high }}$ cells have lower ROS levels than their ALDH ${ }^{\text {low }}$ counterparts (45). Taken together, these results indicate that ALDH1A1 upregulation in response to cytotoxic stress supports CSC survival. Our findings further demonstrated that this process is regulated, at least in part, through sox9 overexpression.

While we focused our attention on ALDH1A1, other ALDH isoforms may contribute to CSC properties in general and to chemotherapy resistance in particular. For example, high ALDH3A1 and ALDH1A3 expression was also reported in lung cancer $(46,47)$. However, unlike ALDH1A1, these isoforms are not a direct target of SOX9 based on our knockdown studies (Figure 4E). We also considered other possible roles of SOX9 in the regulation of cell survival and chemoresistance. It has been suggested that the decreased survival of SOX9 knockdown cells in long-term experiments (i.e. in colony formation or tumor sphere formation assays) may be due to a slower rate of cell proliferation (6). However, SOX9 ${ }^{\text {low }}$ cells were more sensitive to drug treatment even after taking into account the initial effect of knockdown in untreated cells, and the opposite is true for the overexpression experiments (Figure 2D, E, 3E). Furthermore, ectopic expression of SOX9 decreased the rate of cell proliferation while promoting the survival of drug-treated cells (Suppl. Fig. 1D, E, 2C).

In summary, we demonstrated the role of SOX9 in CSC regulation and drug resistance of NSCLC cells. Treatment of the cells with cisplatin upregulates SOX9 
expression, which is crucial to their long-term survival and resistance to chemotherapy. SOX9 mediates its effect on cisplatin resistance through transcriptional activation of ALDH1A1. This novel finding supports the use of SOX9 as a prognostic marker of chemoresistance and as a potential therapeutic target for advanced and recurrent lung cancers. 


\section{Materials and methods}

Cell culture and drugs. H460 (NCI-H460), Beas-2B, HBEC3-KT, H1299, H358, H1650 and A549 cells were purchased from the American Type Culture Collection (ATCC, Manassas, VA, USA) and were passaged less than 15 times. H460 cells were maintained in RPMI medium (Corning, Corning, NY, USA) supplemented with $10 \%$ FBS; A549, H1299, H358, H1650 cells were cultured in DMEM medium (Corning) supplemented with $10 \%$ fetal bovine serum (FBS). Beas-2B and HBEC cells were cultured in BEBM medium supplemented with Clonetics BEGM BulletKit (Lonza, Walkersville, MD, USA). Primary human SAECs (Small Airway Epithelial Cells) immortalized with hTERT were kindly provided by Dr. Tom Hei (48). Primary human SAECs were obtained from Lonza. They were cultured in SABM medium supplemented with Clonetics SAGM BulletKit (Lonza). All cells were maintained in humidified atmosphere at $37{ }^{\circ} \mathrm{C}, 5 \% \quad \mathrm{CO}_{2}$. Cisplatin, etoposide, doxycycline, and 4diethylaminobenzaldehyde (DEAB) were purchased from Sigma-Aldrich Corporation (St. Louis, MO, USA), and paclitaxel from Acros Organics (Geel, Belgium).

Plasmids and generation of stable cell lines. SOX9 siRNA (D-059108-01) and non-targeting siRNA (D-001810-01), pLKO.1 lentiviral plasmids for SOX9 knockdown (\#RHS4533-EG6662) were purchased from Dharmacon (Lafayette, CO, USA). An empty pLKO.1 vector was used as a control and the virus was produced in HEK293T cells (ATCC) as described previously (49). For SOX9 overexpression, SOX9 cDNA from pCMV-AC-GFP-SOX9 (Origene, Rockville, MD, USA) was amplified by PCR and subcloned into pLUTzeo (a gift from Dr. A. Ivanov, West Virginia University, WV, USA). Viral particles were produced as described above, the empty vector was used a control. SiRNA transfections $(50 \mathrm{nM})$ were performed on 6-well dishes with Lipofectamine RNAiMAX transfection reagent (Life Technologies, Carlsbad, CA, USA) according to the manufacturer's protocol. Cells were lysed or used for subsequent experiments 48 hours post-transfection. shRNA and siRNA sequences are listed in the Supplementary table 3.

Immunoblotting. Western blot analysis was performed as described previously (49). Immune complexes were detected by enhanced chemiluminescence in the Amersham Imager 600 (GE Healthcare Life Sciences, Buckinghamshire, UK) and 
quantified with the Amersham Imager software. Antibodies used in this study are listed in the Supplementary table 1.

Flow cytometry. Cells with high aldehyde dehydrogenase activity were identified by staining with the Aldefluor kit (Stem Cell Technologies, Cambridge, MA, USA) according to the manufacturer's instructions. Briefly, for each sample, $3 \times 10^{5}$ cells were incubated in the Aldefluor assay buffer with activated Aldefluor substrate for 45 minutes at $37{ }^{\circ} \mathrm{C}$ in the presence or absence of the specific ALDH inhibitor diethylaminobenzaldehyde (DEAB). Tubes stained with both the Aldefluor substrate and DEAB served as a negative control for each sample. Cells with high ALDH activity $\left(A L D H^{\text {hi }}\right)$ were detected using the BD Fortessa cell analyzer and analyzed with the BD FACSDiva software (BD Biosciences, San Jose, CA, USA). The flow cytometry gates were set to obtain $0.1 \% \mathrm{ALDH}^{\text {hi }}$ cells in substrate + inhibitor tubes for each sample. All experiments were performed at least 3 times.

Cell proliferation assay. The rate of cell proliferation was measured by seeding equal numbers of cells onto 6-well plates in duplicates and counting the cells with the Countess automated cell counter (Life Technologies) every 2 days until the plates become confluent.

Cell viability (MTT) assay. Cells were plated onto 96-well plates and allowed to recover overnight. Next day, cell culture media were replaced with drug-containing media. $10 \mu \mathrm{l}$ of $5 \mathrm{mg} / \mathrm{ml}$ MTT (1-(4,5-dimethyl-2-thiazolyl)-3,5-diphenylformazan) (Tokyo Chemical Industry, Portland, OR, USA) were added to each well containing $100 \mu \mathrm{l}$ of cell culture media following 72 hours of drug treatment. After 2 hours of incubation under standard cell culture conditions, formazan crystals were dissolved with DMSO and absorbance was measured at $570 \mathrm{~nm}$. Cell viability was represented as \% of vehicle-treated values.

Colony formation assay. Cells were treated with cisplatin for 2 days under standard cell culture conditions and allowed to recover without cisplatin for 4 days. The cells (500 cells/well) were then plated onto 6-well plates and cultured under standard conditions for 10 days. Colonies were fixed in 100\% methanol and stained with $0.5 \%$ crystal violet solution. Colonies containing over 50 cells were counted using an Olympus MVX10 microscope (Olympus Corporation, Tokyo, Japan) at $0.63 x$ magnification. The 
colony numbers were normalized to those of respective untreated cells. All experiments were performed in triplicates.

Tumor sphere formation assay. 1,000 cells/well were plated onto ultra-low attachment 24 -well plates (Corning) in $0.8 \%$ methylcellulose (MC)-based serum-free medium (Stem Cell Technologies, \#H4100) supplemented with $20 \mathrm{ng} / \mathrm{ml}$ epidermal growth factor (BD Biosciences), $10 \mathrm{ng} / \mathrm{ml}$ basic fibroblast growth factor (Sigma-Aldrich), and $5 \mathrm{mg} / \mathrm{ml}$ insulin (Sigma-Aldrich). The number of tumor spheres exceeding $50 \mu \mathrm{m}$ in diameter was quantified under a light microscope after 10 days ( $\mathrm{H} 460$ cells) or 3 weeks (A549 cells) in culture. All experiments were performed in triplicates and repeated at least twice, with 5 fields of view analyzed for each replicate. For cisplatin treatments, cisplatin was added to the cultures at the plating time and was replenished 2 times a week thereafter.

Chromatin immunoprecipitation (ChIP). ChIP was carried out using the SimpleChIP kit with agarose beads (Cell Signaling Technology, Danvers, MA, USA) according to the manufacturer's protocol. Briefly, approximately $1 \times 10^{7} \mathrm{H} 460$ cells were used for each immunoprecipitation. Cells were cross-linked with $1 \%$ formaldehyde, treated with micrococcal nuclease for chromatin digestion, and briefly sonicated to break nuclear membranes. $2 \mu \mathrm{g}$ of anti-SOX9 antibody (AB5535, Millipore, Burlington, MA, USA) and negative control normal rabbit IgG (Cell Technologies) were used for immunoprecipitation. The amount of total and ChIP DNA was quantified by qPCR after reversal of cross-links and DNA purification. Primer sequences are listed in the Supplementary table 2 .

Luciferase reporter assay. ALDH1A1 promoter was obtained from SwitchGear Genomics (Carlsbad, CA, USA), and was moved from pLightSwitch vector to pBV-luc (a gift from Bert Vogelstein, Addgene plasmid \# 16539) by restriction enzyme cloning. Empty pBV-luc vector was used as a control. The Renilla luciferase vector pIS1 (a gift from David Bartel, Addgene plasmid \# 12179) served as an internal control for transfection efficiency. SOX9 expression in $\mathrm{H} 460$ cells was induced by treating the cells with $1 \mu \mathrm{g} / \mathrm{ml}$ doxycycline for 5 days before transfection. Cells were transfected on 12 well plates with pBV-luc or pBV-luc-ALDH1A1 (1 $\mu \mathrm{g} /$ well) and $50 \mathrm{ng}$ of plS1 using Viafect (Promega, Madison, WI, USA). Luciferase activities were measured using Dual 
Luciferase Reporter Assay (Promega) according to manufacturer's instructions. HEK293 cells were transfected with the luciferase plasmids as above plus pcDNA3.1RFP or pcDNA3.1-SOX9.

RT-qPCR. Total RNA was purified using the RNeasy kit (Qiagen, Germantown MD, USA). Reverse transcription was performed with SuperScript III Reverse Transcriptase (Life Technologies) using oligo(dT) primers. Quantitative real-time PCR was carried out using SYBR Green Master Mix (Applied Biosystems, Foster City, CA) in a Step One Plus PCR Cycler (Applied Biosystems). Results were calculated using the $2^{-\triangle \Delta C t}$ method; glyceraldehyde-3-phosphate dehydrogenase (GAPDH) and $\beta$-actin served as the internal controls. Primer sequences are listed in the Supplementary table 2.

Statistical analysis. The data represent means \pm SD or SEM as indicated. Statistical comparisons were made using two-tailed Student's $t$ test or ANOVA when more than two groups were analyzed. Statistical analysis was performed with GraphPad Prism software (GraphPad, La Jolla, CA, USA), and $p<0.05$ was considered statistically significant. 


\section{Acknowledgements}

The authors wish to thank Dr. Alexey Ivanov for providing pLUTz cloning vector.

\section{Funding}

This work was supported in part by the National Institutes of Health grants R01ES022968 and R01-EB018857.

Imaging experiments and image analysis were performed in the West Virginia University Microscope Imaging Facility, which has been supported by the WVU Cancer Institute and the National Institutes of Health grants P20RR016440, P20GM103434 and P30RR032138/P30GM103488.

Flow Cytometry experiments were performed in the West Virginia University Flow Cytometry \& Single Cell Core Facility, which is supported by the National Institutes of Health equipment grant S10OD016165 and the Institutional Development Awards (50)

from the National Institute of General Medical Sciences of the National Institutes of Health under grant numbers P30GM103488 (Cancer CoBRE) and P20GM103434 (INBRE).

\section{Competing Interests}

The authors declare that they have no conflict of interests.

\section{Disclaimer:}

The findings and conclusions in this report are those of the author(s) and do not necessarily represent the official position of the National Institute for Occupational Safety and Health, Centers for Disease Control and Prevention. 
Figures and legends

A

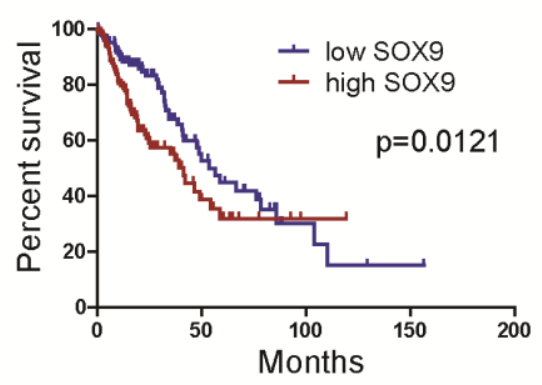

C

Normal epithelial cells Lung cancer cells

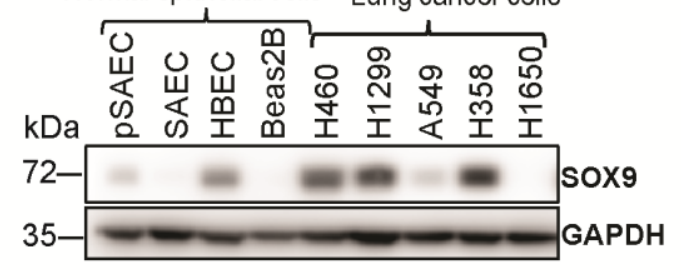

F

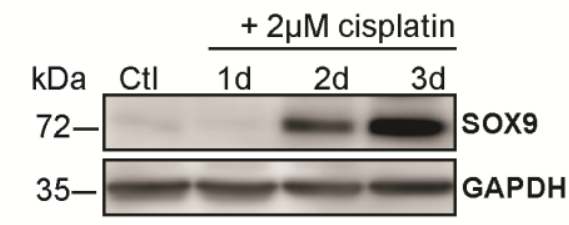

D
B

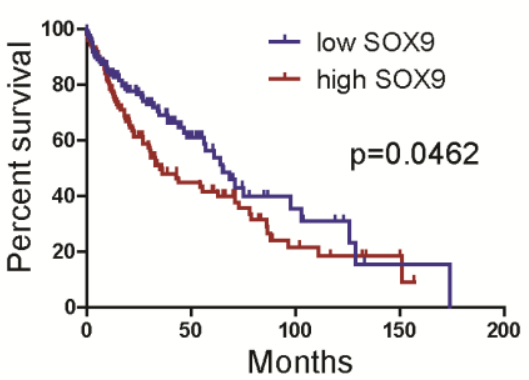

E

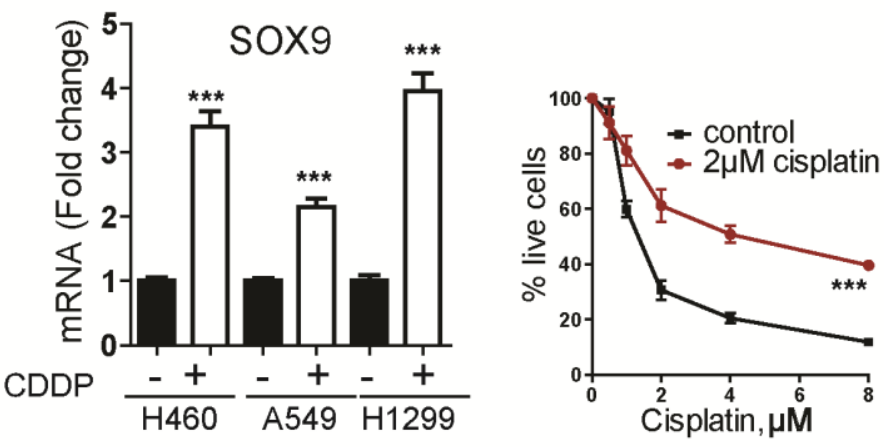

Figure 1. 
Figure 1. High SOX9 expression correlates with poor survival in NSCLC patients and is induced by cisplatin in lung cancer cells. (A, B) Overall survival in NSCLC patients from the TCGA database. Patients with adenocarcinoma $(n=260)(\mathbf{A})$ and squamous cell carcinoma $(n=251)$ (B) with high SOX9 expression (top $25 \%$ expression vs. bottom 25\%) had a lower overall survival. P values by log-rank (Mantel-Cox) test. (C) Western blot analysis of SOX9 expression in normal lung epithelial cells and lung cancer cell lines. (D) SOX9 mRNA levels are increased after a 3-day long treatment with cisplatin ( $2 \mu \mathrm{M}$ for H460, $8 \mu \mathrm{M}$ for A549 and H1299 cells). Data are mean $\pm \mathrm{SD}$, Representative of 3 independent experiments, $p<0.0001$ by one-way ANOVA; Tukey's multiple comparison test. (E) $\mathrm{H} 460$ cells became resistant to cisplatin after a 3-day exposure to $2 \mu \mathrm{M}$ cisplatin $\left(\mathrm{IC}_{50} 4.3 \mu \mathrm{M}\right.$ vs. $\left.1.4 \mu \mathrm{M}\right)$. Cisplatin resistant cells were treated with various cisplatin concentrations for 72 hours and cell viability was assessed by MTT assay. Representative of 3 independent experiments, 5 technical replicates each. Data are mean $\pm S D, p<0.0001$ by two-way repeated measures ANOVA. (F) Representative Western blot showing SOX9 upregulation in $\mathrm{H} 460$ cells in response to cisplatin treatment, $\mathrm{n}=3$ independent experiments. CDDP - cisplatin. 
A

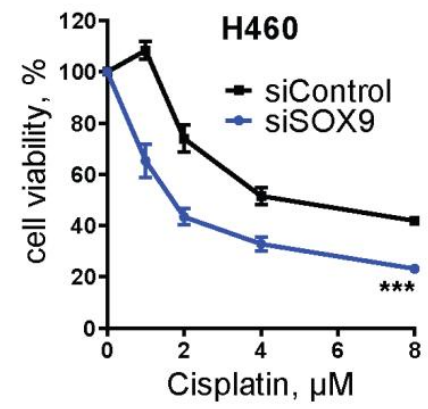

C

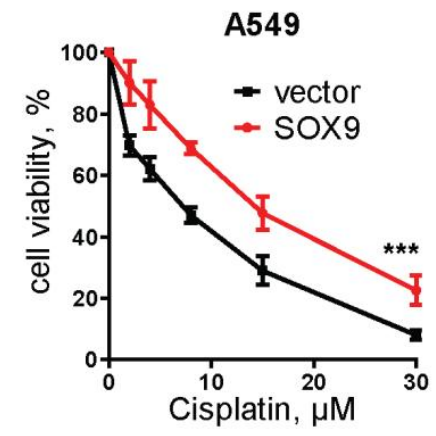

E

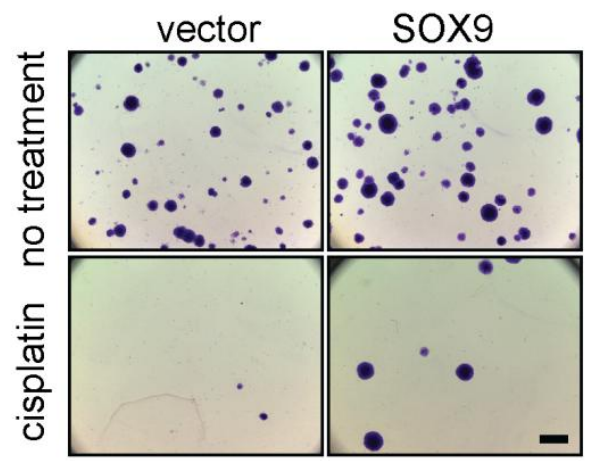

D
B
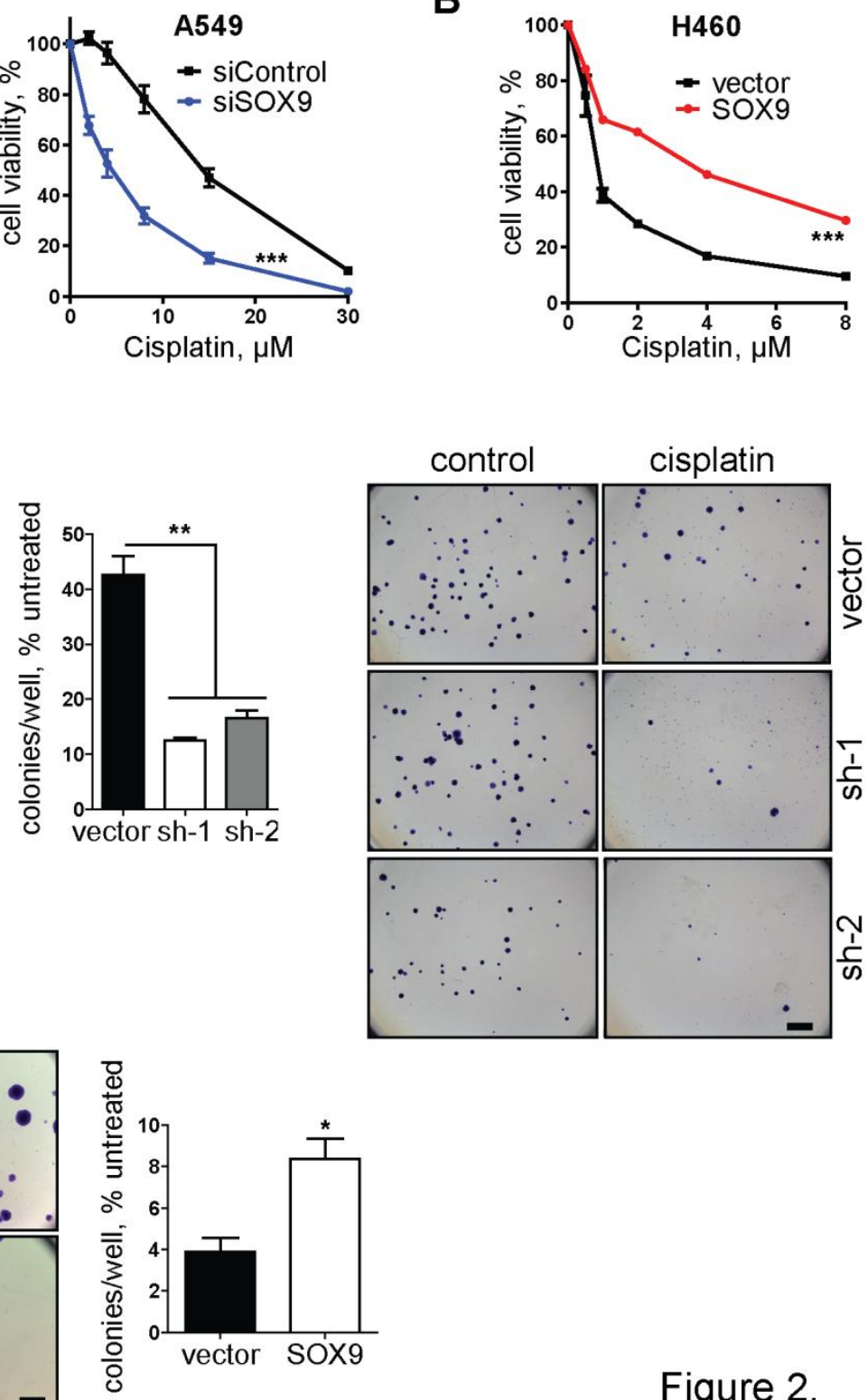

Figure 2. 
Figure 2. SOX9 overexpression promotes chemoresistance in NSCLC cells. (A) Dose-response curves of $\mathrm{H} 460$ and A549 cells transfected with control and SOX9 siRNA. Cell viability was evaluated by MTT assay following a 3-day cisplatin treatment. Representative of 3 independent experiments, 4 technical replicates in each. Data are mean \pm SD. (B) Dose-response curve of empty vector and SOX9 overexpressing H460 cells following a 3-day cisplatin treatment. SOX9 expression was induced by doxycycline treatment 5 days prior to the experiment. Representative of 5 independent experiments, 5 technical replicates in each. Data are mean \pm SD. (C) Same as in (B) in A549 cells. (D) Colony formation assay in vector control and SOX9 knockdown H460 cells. Cells were treated with $4 \mu \mathrm{M}$ of cisplatin for 2 days, off cisplatin for 4 days, and allowed to form colonies for 10 days. The cells were then fixed, stained with crystal violet, and visualized by light microscopy. Data are mean $\pm S D$, scale bar $=3 \mathrm{~mm}, \mathrm{n}=2$ biological replicates. $p=0.0021$ by one-way ANOVA; Tukey's multiple comparison test. (E) Same as in (D) with SOX9 overexpressing cells exposed to $2 \mu \mathrm{M}$ of cisplatin. $n=3$ biological replicates, $p=0.0125$ by unpaired two-tailed $t$ test. ${ }^{* * *}-p<0.0001$ by twoway ANOVA. 
A
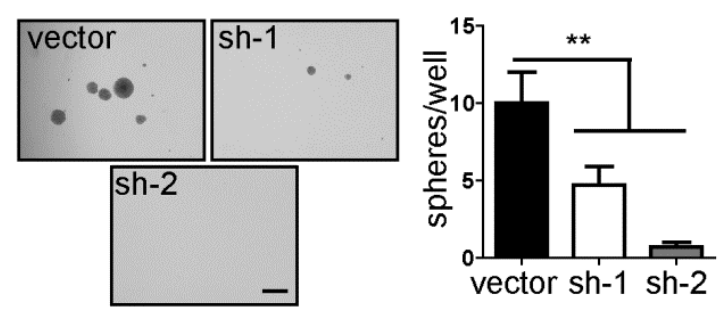

D

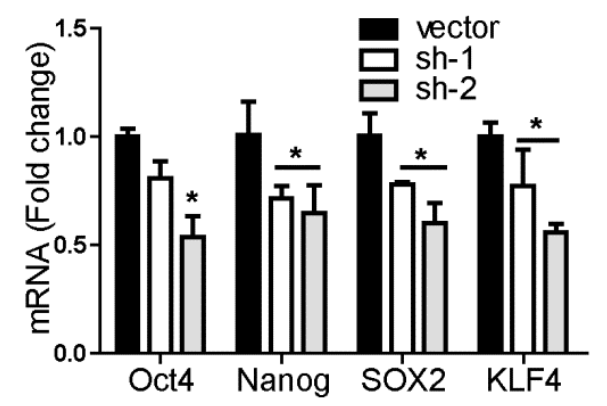

B
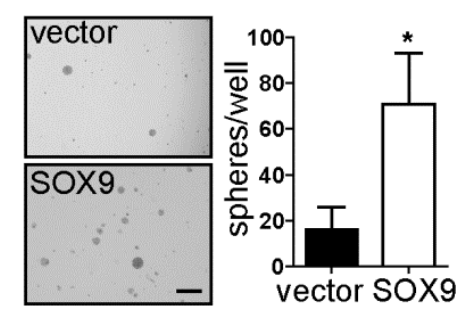

C

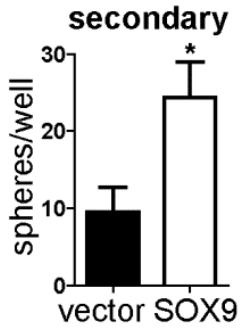

$\mathbf{E}$

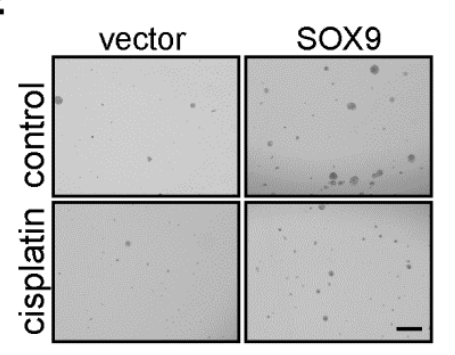

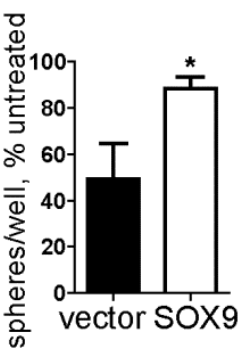

Figure 3.

Figure 3. SOX9 promotes cancer stem-like properties. (A) Tumor sphere formation in H460 cells expressing an empty vector or each of the two shRNAs against SOX9, $n=$ 3 biological replicates. Data are mean $\pm S D ; p<0.0001$ by one-way ANOVA; Tukey's multiple comparison test. Scale bar $=500 \mu \mathrm{m}$. (B, C) Same as in (A) with the empty vector and SOX9 overexpressing cells, and secondary tumor spheres formed by the cells from primary spheres (C). Means $\pm S D$ are shown; $p=0.005$ for primary spheres, $p=0.012$ for secondary spheres by unpaired two-tailed $t$ test, $n=3$ biological replicates. (D) Decreased mRNA expression of stem-cell markers in SOX9 knockdown cells (sh-1, sh-2) vs. control vector. The data are presented as mean $\pm S D,{ }^{*} p<0.05$ comparing to vector, two-way ANOVA followed by Bonferroni posttests. (E) As in (B) plus cells were exposed to $0.25 \mu \mathrm{M}$ of cisplatin for the duration of the experiment, $p=0.043$ by unpaired two-tailed t test, $\mathrm{n}=3$ biological replicates. All experiments were repeated at least 2 times. 
A

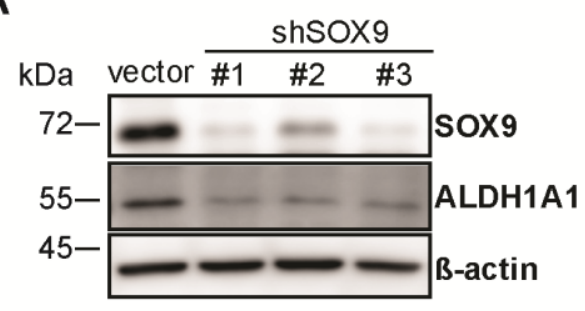

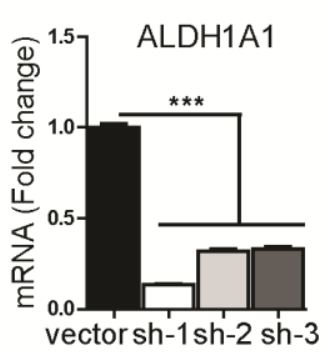

B

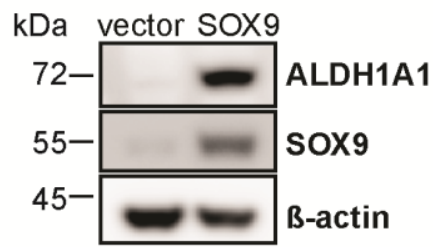

C
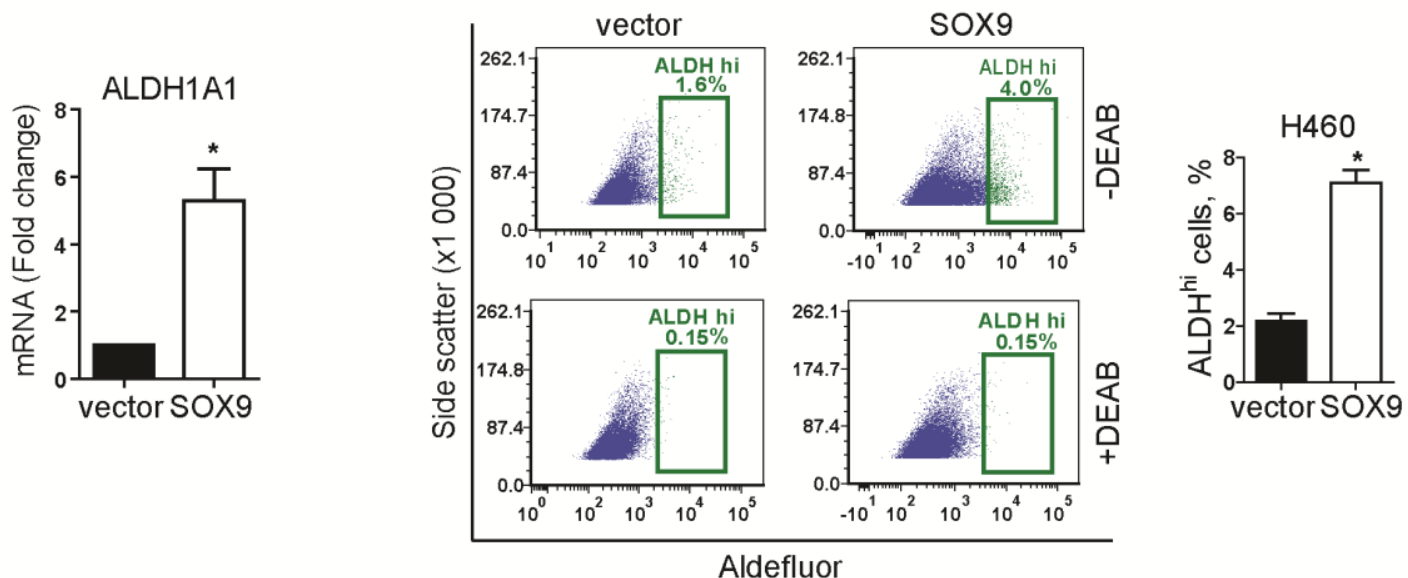

E
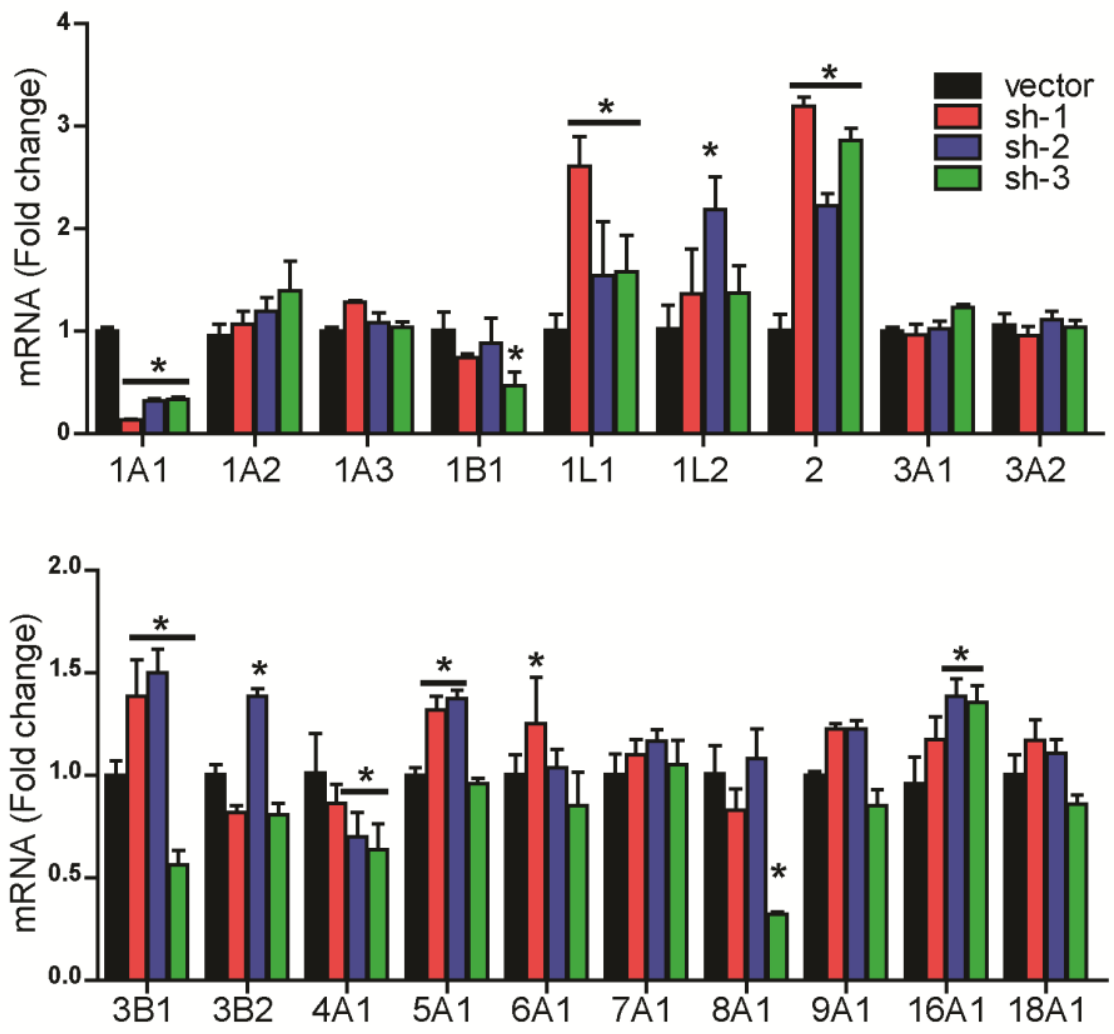

Figure 4. 
Figure 4. ALDH1A1 is a downstream target of SOX9. (A) Left - representative Western blot analysis of SOX9 and ALDH1A1 expression in SOX9 knockdown (sh 1, 2, 3) and empty vector H460 cells. Right - ALDH1A1 mRNA levels in same cells. Data are mean \pm SEM, $n=3, p<0.0001$ by one-way ANOVA; Tukey's multiple comparison test. (B) Representative Western blot showing an increased expression of ALDH1A1 in SOX9 overexpressing $\mathrm{H} 460$ cells, $n=6$ independent experiments. (C) SOX9 overexpression induced ALDH1A1 mRNA levels in H460 cells. Data are mean $\pm S D, n=$ 7 independent experiments, $p=0.004$ by paired t test. (D) Analysis of ALDH activity by Aldefluor assay in empty vector and SOX9 overexpressing H460 cells. Left - a representative flow cytometry gating for Aldefluor assay. Right - \% of cells with high ALDH activity, $n=3$ independent experiments, 2 biological replicates each. Data are mean $\pm S D, p<0.001$, paired two-tailed t-test. DEAB - N, N-diethylaminobenzaldehyde, ALDH inhibitor, used to set up background fluorescence level for all flow cytometry experiments. (E) RT-qPCR analysis of 19 ALDH isoforms in SOX9 knockdown (sh-1, -2, -3 ) and empty vector $\mathrm{H} 460$ cells. Data are mean $\pm \mathrm{SD}$, * $p<0.05$ comparing to vector, two-way ANOVA followed by Bonferroni posttests. 
A

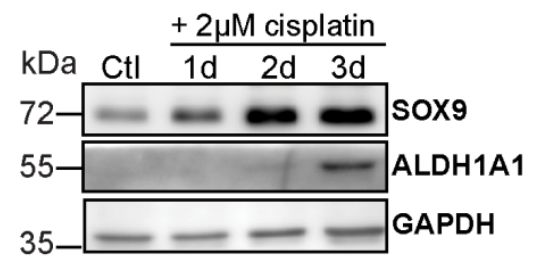

Figure 5.
B

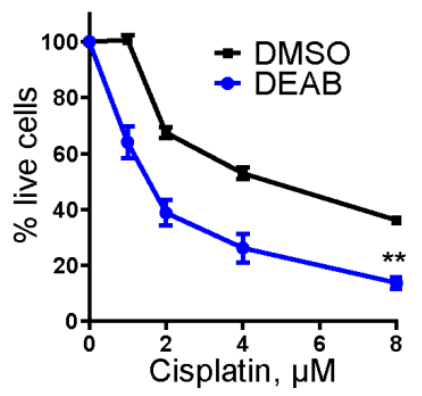

C

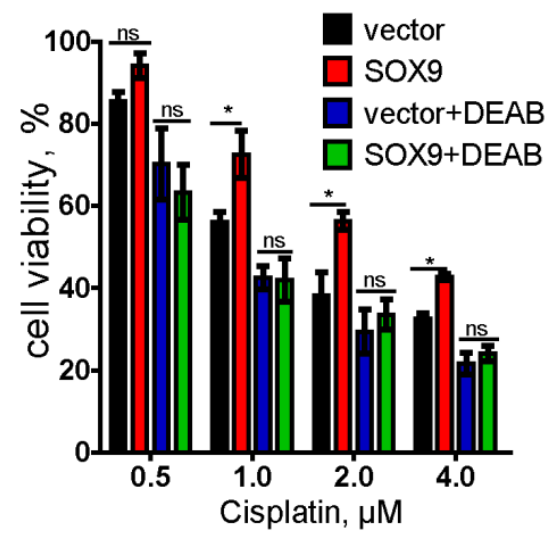

Figure 5. SOX9 promotes chemoresistance through ALDH1A1. (A) Representative western blot analysis of SOX9 and ALDH1A1 expression in $\mathrm{H} 460$ cells after exposure to $2 \mu \mathrm{M}$ cisplatin. (B) Treatment with ALDH inhibitor DEAB enhances sensitivity to cisplatin. H460 cells were pretreated with $200 \mu \mathrm{M}$ DEAB or DMSO for $24 \mathrm{~h}$, followed by cisplatin treatment and analyzed for cell viability after 3 days by MTT assay. DEAB decreased $\mathrm{IC}_{50}$ of $\mathrm{H} 460$ cells $2.5 \pm 0.3$ folds, $\mathrm{n}=5$ independent experiments, $\mathrm{p}=0.0110$ by paired t test. (C) ALDH inhibitor DEAB reverses the effect of SOX9 overexpression in H460 cells. Cisplatin dose-response curve evaluated by MTT assay. Data are mean \pm SD, two-way ANOVA with Bonferroni posttests. All experiments were repeated at least 3 times. ns - not significant. 
A

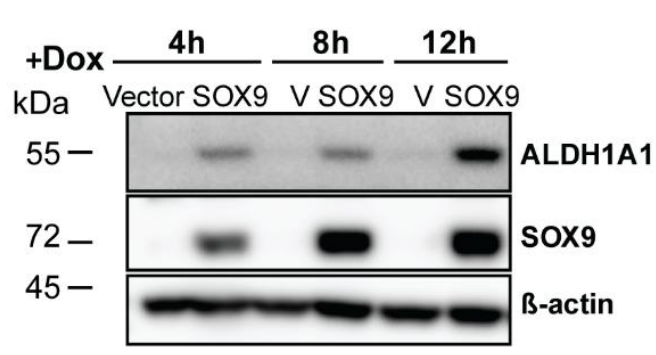

C

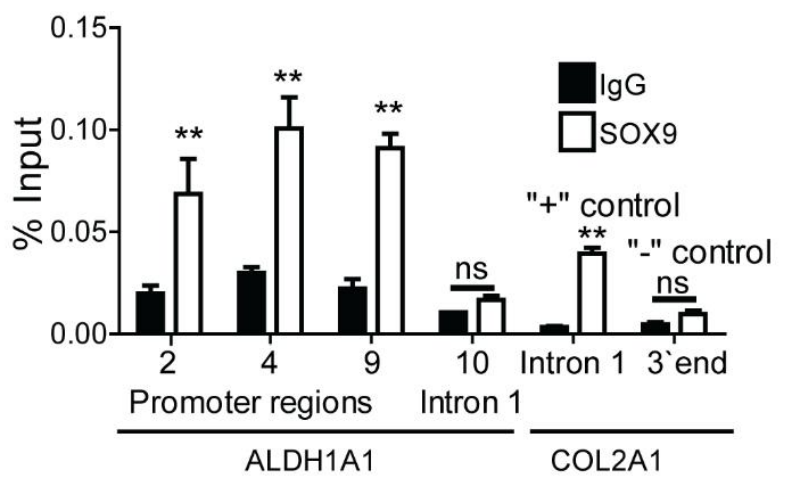

B

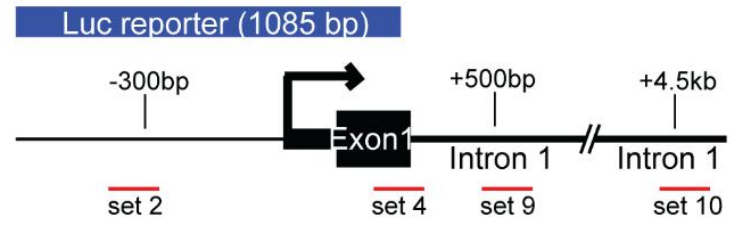

D

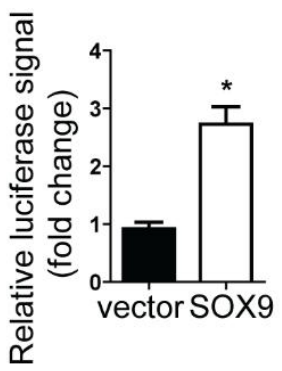

Figure 6. ALDH1A1 is regulated by SOX9 at the transcriptional level. (A) SOX9 overexpression induced by doxycycline for the indicated length of time stimulates ALDH1A1 expression. The blots shown are representative of two independent experiments. (B) Schematic image of the ALDH1A1 promoter and locations of primer sets used in (C). (C) SOX9 binding is enriched at the ALDH1A1 promoter. SOX9 ChIP results were analyzed by qPCR with primers specific to the indicated DNA regions. IgG was used as a negative IP control. Primers to COL2A1 served as negative and positive controls for ChIP. Data are mean $\pm S D,{ }^{* *}-p<0.01$, two-way ANOVA with Bonferroni posttests. ns - not significant. (D) Luciferase reporter assay with the ALDH1A1 promoter. H460 cells stably expressing empty vector or SOX9 were transfected with ALDH1A1 luciferase reporter plasmid. Luciferase activity was analyzed 48 hours posttransfection and normalized to Renilla luciferase signal. $p=0.028$ by unpaired twotailed $t$ test. Bars are mean $\pm S E M, n=2$ independent experiments. 


\section{References}

1. Jo A, Denduluri S, Zhang B, Wang Z, Yin L, Yan Z, et al. The versatile functions of Sox9 in development, stem cells, and human diseases. Genes Dis. 2014;1(2):149-61.

2. Furuyama K, Kawaguchi $\mathrm{Y}$, Akiyama H, Horiguchi M, Kodama S, Kuhara T, et al. Continuous cell supply from a Sox9-expressing progenitor zone in adult liver, exocrine pancreas and intestine. Nat Genet. 2011;43(1):34-41.

3. Scott CE, Wynn SL, Sesay A, Cruz C, Cheung M, Gomez Gaviro MV, et al. SOX9 induces and maintains neural stem cells. Nat Neurosci. 2010;13(10):1181-9.

4. Rockich BE, Hrycaj SM, Shih HP, Nagy MS, Ferguson MA, Kopp JL, et al. Sox9 plays multiple roles in the lung epithelium during branching morphogenesis. Proc Natl Acad Sci USA. 2013;110(47):E4456-64.

5. Li L, Zhang H, Min D, Zhang R, Wu J, Qu H, et al. Sox9 activation is essential for the recovery of lung function after acute lung injury. Cell Physiol Biochem. 2015;37(3):1113-22.

6. Matheu A, Collado M, Wise C, Manterola L, Cekaite L, Tye AJ, et al. Oncogenicity of the developmental transcription factor Sox9. Cancer Res. 2012;72(5):1301-15.

7. Ruan H, Hu S, Zhang H, Du G, Li X, Li X, et al. Upregulated SOX9 expression indicates worse prognosis in solid tumors: a systematic review and meta-analysis. Oncotarget. 2017;8(68):113163-73.

8. Lei B, Zhang YX, Liu T, Li YW, Pang D. Sox9 upregulation in breast cancer is correlated with poor prognosis and the CD44(+)/CD24(-/low) phenotype. Int J Clin Exp Pathol. 2016;9(7):7345-51.

9. Jordan CT, Guzman ML, Noble M. Cancer stem cells. N Engl J Med. 2006;355(12):1253-61.

10. Larsimont JC, Youssef KK, Sanchez-Danes A, Sukumaran V, Defrance M, Delatte B, et al. Sox9 Controls Self-Renewal of Oncogene Targeted Cells and Links Tumor Initiation and Invasion. Cell Stem Cell. 2015;17(1):60-73.

11. Liu C, Liu L, Chen X, Cheng J, Zhang H, Shen J, et al. Sox9 Regulates Self-Renewal and Tumorigenicity by Promoting Symmetrical Cell Division of Cancer Stem Cells in Hepatocellular Carcinoma. Hepatology. 2016.

12. Luanpitpong S, Li J, Manke A, Brundage K, Ellis E, McLaughlin SL, et al. SLUG is required for SOX9 stabilization and functions to promote cancer stem cells and metastasis in human lung carcinoma. Oncogene. 2016;35(22):2824-33.

13. Santos JC, Carrasco-Garcia E, Garcia-Puga M, Aldaz P, Montes M, Fernandez-Reyes $\mathrm{M}$, et al. SOX9 Elevation Acts with Canonical WNT Signaling to Drive Gastric Cancer Progression. Cancer Res. 2016.

14. Hong X, Liu W, Song R, Shah JJ, Feng X, Tsang CK, et al. SOX9 is targeted for proteasomal degradation by the E3 ligase FBW7 in response to DNA damage. Nucleic Acids Res. 2016;44(18):8855-69.

15. Suryo Rahmanto A, Savov V, Brunner A, Bolin S, Weishaupt H, Malyukova A, et al. FBW7 suppression leads to SOX9 stabilization and increased malignancy in medulloblastoma. EMBO J. 2016;35(20):2192-212.

16. Higashihara T, Yoshitomi H, Nakata Y, Kagawa S, Takano S, Shimizu H, et al. Sex Determining Region Y Box 9 Induces Chemoresistance in Pancreatic Cancer Cells by Induction of Putative Cancer Stem Cell Characteristics and Its High Expression Predicts Poor Prognosis. Pancreas. 2017;46(10):1296-304.

17. Zhou CH, Ye LP, Ye SX, Li Y, Zhang XY, Xu XY, et al. Clinical significance of SOX9 in human non-small cell lung cancer progression and overall patient survival. J Exp Clin Cancer Res. 2012;31. 
18. Jiang SS, Fang WT, Hou YH, Huang SF, Yen BL, Chang JL, et al. Upregulation of SOX9 in Lung Adenocarcinoma and Its Involvement in the Regulation of Cell Growth and Tumorigenicity. Clin Cancer Res. 2010;16(17):4363-73.

19. Barr MP, Gray SG, Hoffmann AC, Hilger RA, Thomale J, O'Flaherty JD, et al. Generation and characterisation of cisplatin-resistant non-small cell lung cancer cell lines displaying a stem-like signature. PLoS One. 2013;8(1):e54193.

20. Dallas NA, Xia L, Fan F, Gray MJ, Gaur P, van Buren G, 2nd, et al. Chemoresistant colorectal cancer cells, the cancer stem cell phenotype, and increased sensitivity to insulin-like growth factor-I receptor inhibition. Cancer Res. 2009;69(5):1951-7.

21. Levina V, Marrangoni AM, DeMarco R, Gorelik E, Lokshin AE. Drug-selected human lung cancer stem cells: cytokine network, tumorigenic and metastatic properties. PLoS One. 2008;3(8):e3077.

22. Dean M, Fojo T, Bates S. Tumour stem cells and drug resistance. Nat Rev Cancer. 2005;5(4):275-84.

23. Dontu G, Abdallah WM, Foley JM, Jackson KW, Clarke MF, Kawamura MJ, et al. In vitro propagation and transcriptional profiling of human mammary stem/progenitor cells. Genes Dev. 2003;17(10):1253-70.

24. Eramo A, Lotti F, Sette G, Pilozzi E, Biffoni M, Di Virgilio A, et al. Identification and expansion of the tumorigenic lung cancer stem cell population. Cell Death Differ. 2008;15(3):504-14.

25. Wernig M, Meissner A, Foreman R, Brambrink T, Ku M, Hochedlinger K, et al. In vitro reprogramming of fibroblasts into a pluripotent ES-cell-like state. Nature. 2007;448(7151):31824.

26. Marchitti SA, Brocker C, Stagos D, Vasiliou V. Non-P450 aldehyde oxidizing enzymes: the aldehyde dehydrogenase superfamily. Expert Opin Drug Metab Toxicol. 2008;4(6):697-720.

27. Pors K, Moreb JS. Aldehyde dehydrogenases in cancer: an opportunity for biomarker and drug development? Drug Discov Today. 2014;19(12):1953-63.

28. Wei Y, Wu S, Xu W, Liang Y, Li Y, Zhao W, et al. Depleted aldehyde dehydrogenase 1A1 (ALDH1A1) reverses cisplatin resistance of human lung adenocarcinoma cell A549/DDP. Thorac Cancer. 2017;8(1):26-32.

29. Januchowski R, Wojtowicz K, Sterzyska K, Sosiska P, Andrzejewska M, Zawierucha P, et al. Inhibition of ALDH1A1 activity decreases expression of drug transporters and reduces chemotherapy resistance in ovarian cancer cell lines. Int J Biochem Cell Biol. 2016;78:248-59.

30. Tomita $\mathrm{H}$, Tanaka K, Tanaka T, Hara A. Aldehyde dehydrogenase $1 \mathrm{~A} 1$ in stem cells and cancer. Oncotarget. 2016;7(10):11018-32.

31. Morgan CA, Parajuli B, Buchman CD, Dria K, Hurley TD. N,N-diethylaminobenzaldehyde (DEAB) as a substrate and mechanism-based inhibitor for human ALDH isoenzymes. Chem Biol Interact. 2015;234:18-28.

32. Marcato P, Dean CA, Pan D, Araslanova R, Gillis M, Joshi M, et al. Aldehyde dehydrogenase activity of breast cancer stem cells is primarily due to isoform ALDH1A3 and its expression is predictive of metastasis. Stem cells (Dayton, Ohio). 2011;29(1):32-45.

33. Ma F, Ye H, He HH, Gerrin SJ, Chen S, Tanenbaum BA, et al. SOX9 drives WNT pathway activation in prostate cancer. J Clin Invest. 2016;126(5):1745-58.

34. Cronin KA, Lake AJ, Scott S, Sherman RL, Noone AM, Howlader N, et al. Annual Report to the Nation on the Status of Cancer, part I: National cancer statistics. Cancer. 2018;124(13):2785-800.

35. Zhao W, Zang C, Zhang T, Li J, Liu R, Feng F, et al. Clinicopathological characteristics and prognostic value of the cancer stem cell marker ALDH1 in ovarian cancer: a meta-analysis. Onco Targets Ther. 2018;11:1821-31. 
36. Li J, Zhang B, Yang YF, Jin J, Liu YH. Aldehyde dehydrogenase 1 as a predictor of the neoadjuvant chemotherapy response in breast cancer: A meta-analysis. Medicine. 2018;97(34):e12056.

37. Ajani JA, Wang X, Song S, Suzuki A, Taketa T, Sudo K, et al. ALDH-1 expression levels predict response or resistance to preoperative chemoradiation in resectable esophageal cancer patients. Mol Oncol. 2014;8(1):142-9.

38. Huo W, Du M, Pan X, Zhu X, Li Z. Prognostic value of ALDH1 expression in lung cancer: a meta-analysis. Int J Clin Exp Med. 2015;8(2):2045-51.

39. Wei D, Peng J-J, Gao H, Zhang T, Tan Y, Hu Y-H. ALDH1 Expression and the Prognosis of Lung Cancer: A Systematic Review and Meta-Analysis. Heart Lung Circ. 2015;24(8):780-8.

40. Chen J, Xia Q, Jiang B, Chang W, Yuan W, Ma Z, et al. Prognostic Value of Cancer Stem Cell Marker ALDH1 Expression in Colorectal Cancer: A Systematic Review and MetaAnalysis. PLoS One. 2015;10(12):e0145164.

41. Zhou C, Sun B. The prognostic role of the cancer stem cell marker aldehyde dehydrogenase 1 in head and neck squamous cell carcinomas: a meta-analysis. Oral Oncol. 2014;50(12):1144-8.

42. Duong HQ, Hwang JS, Kim HJ, Kang HJ, Seong YS, Bae I. Aldehyde dehydrogenase $1 \mathrm{~A} 1$ confers intrinsic and acquired resistance to gemcitabine in human pancreatic adenocarcinoma MIA PaCa-2 cells. Int J Oncol. 2012;41(3):855-61.

43. von Eitzen U, Meier-Tackmann D, Agarwal DP, Goedde HW. Detoxification of cyclophosphamide by human aldehyde dehydrogenase isozymes. Cancer Lett. 1994;76(1):459.

44. Moreb J, Zucali JR, Zhang Y, Colvin MO, Gross MA. Role of aldehyde dehydrogenase in the protection of hematopoietic progenitor cells from 4-hydroperoxycyclophosphamide by interleukin 1 beta and tumor necrosis factor. Cancer Res. 1992;52(7):1770-4.

45. Mizuno T, Suzuki N, Makino H, Furui T, Morii E, Aoki H, et al. Cancer stem-like cells of ovarian clear cell carcinoma are enriched in the ALDH-high population associated with an accelerated scavenging system in reactive oxygen species. Gynecol Oncol. 2015;137(2):299305.

46. Patel M, Lu L, Zander DS, Sreerama L, Coco D, Moreb JS. ALDH1A1 and ALDH3A1 expression in lung cancers: correlation with histologic type and potential precursors. Lung Cancer. 2008;59(3):340-9.

47. Shao C, Sullivan JP, Girard L, Augustyn A, Yenerall P, Rodriguez-Canales J, et al. Essential role of aldehyde dehydrogenase $1 \mathrm{~A} 3$ for the maintenance of non-small cell lung cancer stem cells is associated with the STAT3 pathway. Clin Cancer Res. 2014;20(15):415466.

48. Piao CQ, Liu L, Zhao YL, Balajee AS, Suzuki M, Hei TK. Immortalization of human small airway epithelial cells by ectopic expression of telomerase. Carcinogenesis. 2005;26(4):725-31.

49. Voronkova MA, Luanpitpong S, Rojanasakul LW, Castranova V, Dinu CZ, Riedel H, et al. SOX9 Regulates Cancer Stem-Like Properties and Metastatic Potential of Single-Walled Carbon Nanotube-Exposed Cells. Sci Rep. 2017;7(1):11653.

50. Umeyama H, Iwadate M, Taguchi Yh. TINAGL1 and B3GALNT1 are potential therapy target genes to suppress metastasis in non-small cell lung cancer. BMC Genomics. 2014;15 Suppl 9:S2. 


\section{Supplementary materials}

A

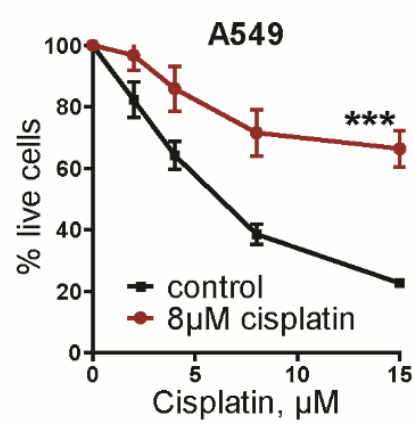

C

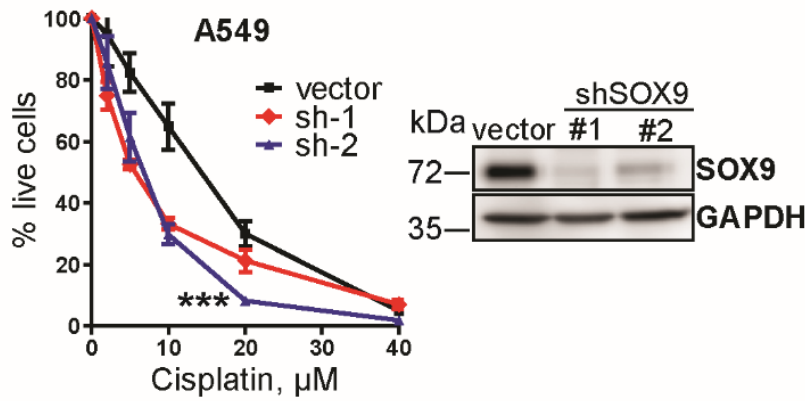

B

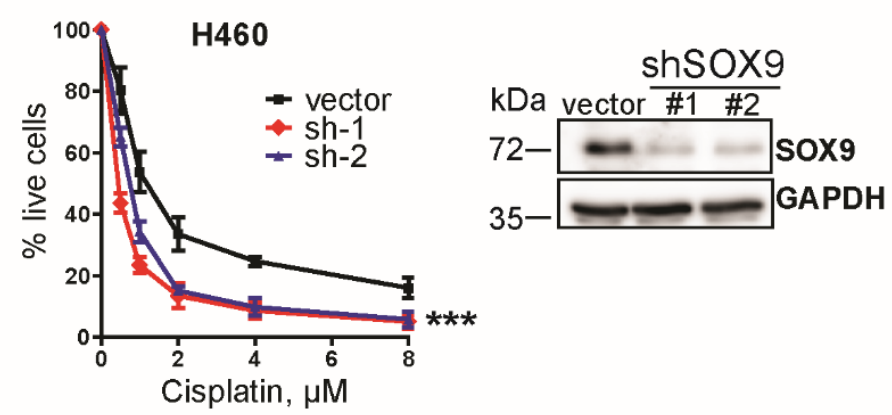

D

E
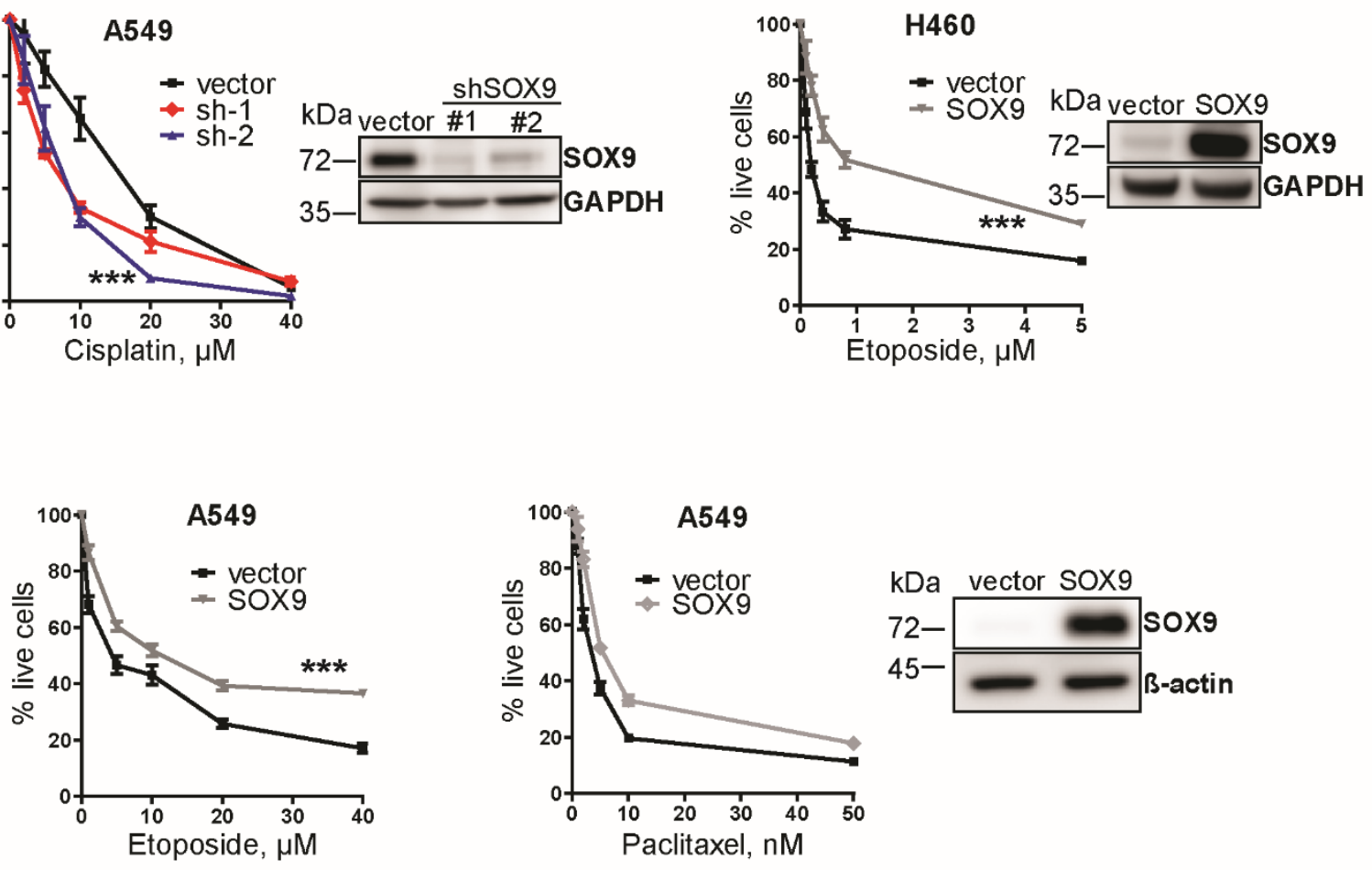

E 


\section{Supplementary Figure 1. SOX9 expression levels correlate with drug sensitivity in}

NSCLC cells. (A) A549 cells exposed to $8 \mu \mathrm{M}$ cisplatin for 3 days become less sensitive to the chemodrug (IC50 $27.8 \mu \mathrm{M}$ vs. $6.0 \mu \mathrm{M}$ ). Mean \pm SD, the experiment was repeated 3 times. Cell viability was measured by MTT assay. (B) SOX9 knockdown renders $\mathrm{H} 460$ cells sensitive to cisplatin. Results are shown as mean \pm SD. A representative western blot image demonstrates SOX9 knockdown levels generated by 2 shRNAs against SOX9 (sh-1 and sh-2) comparing to vector control. (C) Same as in B in A549 cells. (D) SOX9 overexpression in $\mathrm{H} 460$ cells induces resistance to etoposide. Results are mean $\pm S D$, the experiment was repeated 3 times. A representative western blot image demonstrates levels of SOX9 overexpression. (E) SOX9 overexpression renders A549 cells resistant to etoposide and paclitaxel $(p=0.0036)$. Results are shown as mean \pm SEM. Right - representative western blot analysis of SOX9 protein levels in SOX9 overexpressing A549 cells and vector control cells. ${ }^{* * *}-p<0.0001$ by two-way repeated measures ANOVA followed by Bonferroni posttests. 
A
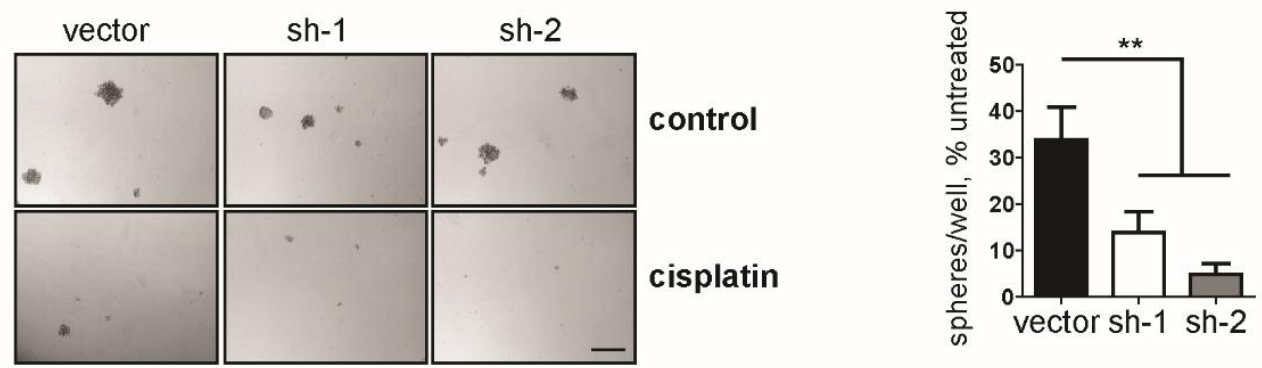

B

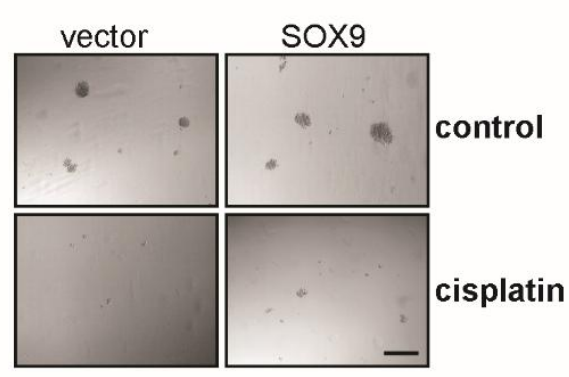

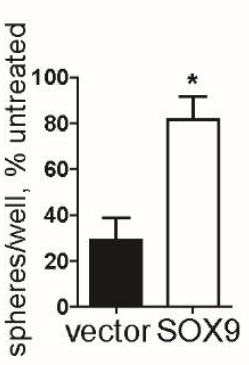

C
A549

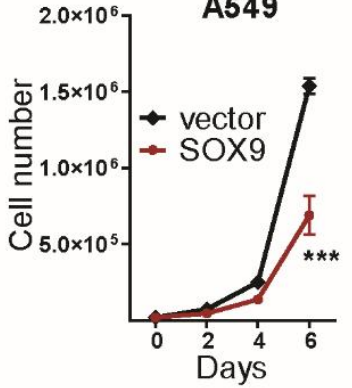

Supplementary Figure 2. SOX9 expression correlates with drug sensitivity in 3-D conditions in A549 cells. (A) Representative pictures of tumor spheres formed by A549 cells expressing the empty vector or shRNAs against SOX9 (sh-1, sh-2) under standard conditions or after cisplatin $(1.5 \mu \mathrm{M})$ treatment. Scale bar $-300 \mu \mathrm{m}$. Right quantification of spheroids after treatment with cisplatin. $p=0.0042$ by one-way ANOVA followed by Tukey's multiple comparison test. Bars are mean $\pm S D, n=3$. (B) Representative pictures of tumor spheres formed by A549 cells expressing the empty vector or SOX9 under standard conditions or after cisplatin $(1.0 \mu \mathrm{M})$ treatment. Scale bar $-300 \mu \mathrm{m}$. Bars are means $\pm S D, n=3 ; p=0.0106$ by unpaired two-tailed $t$ test. (C) SOX9 overexpression slows down cell proliferation in $\mathrm{H} 460$ and A549 cells. Proliferation rate of the indicated cells was analyzed by counting the cells every 2 days, $n=3$ biological replicates. $\mathrm{p}=0.011$ for $\mathrm{H} 460, \mathrm{p}=0.0068$ for A549 cells by two-way repeated measures ANOVA followed by Bonferroni posttests. Means \pm SD are shown. 
A

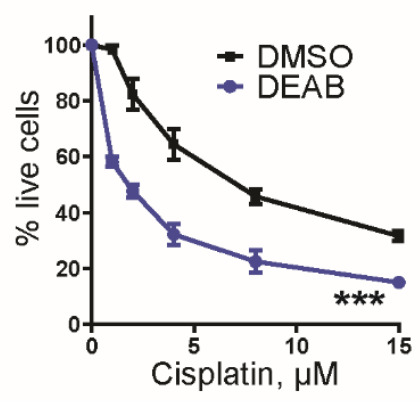

B

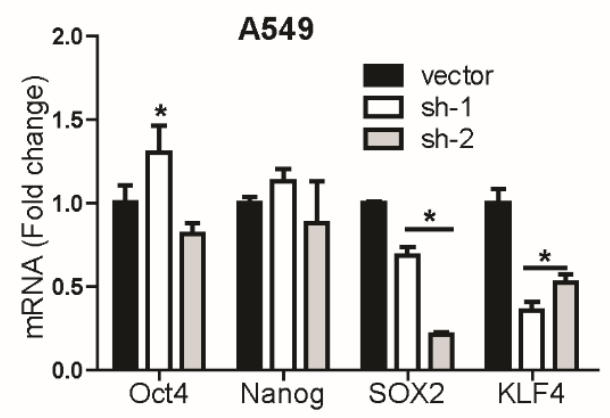

C

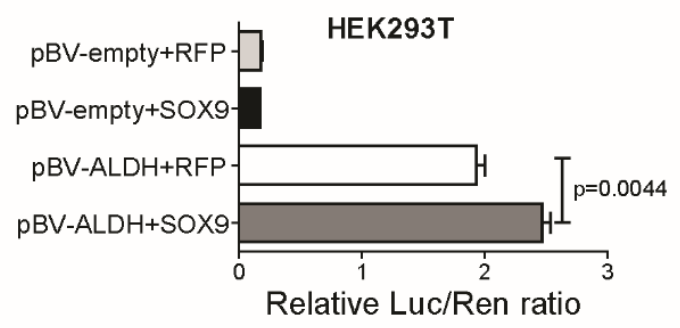

Supplementary Figure 3. (A) Treatment with ALDH inhibitor DEAB enhances sensitivity to cisplatin. $\mathrm{H} 460$ cells were pretreated with $200 \mu \mathrm{M}$ DEAB or DMSO for $24 \mathrm{~h}$, followed by cisplatin treatment and analyzed for cell viability after 2 days by MTT assay. Means $\pm S D$ are shown, $p<0.0001$ by two-way repeated measures ANOVA followed by Bonferroni posttests. (B) Real-time PCR analysis of mRNA levels of indicated stem cell markers in A549 cells expressing the empty vector or shRNAs against SOX9 (sh-1, sh$2)$. Means $\pm S D$ are shown, $p<0.05$ by two-way ANOVA followed by Bonferroni posttests. (C) SOX9 induces ALDH1A1 luciferase reporter activity in HEK293 cells. Cells were transfected with the indicated constructs and luciferase activity was measured $48 \mathrm{~h}$ posttransfection. Bars are mean \pm SEM, $p=0.0044$ by unpaired twotailed $\mathrm{t}$ test. 
Supplementary Table 1. List of antibodies used in this study.

\begin{tabular}{|l|l|l|}
\hline Name & Company & Catalog \# \\
\hline SOX9 & EMD Millipore & AB5535 \\
\hline ALDH1A1 & EMD Millipore & MABN838 \\
\hline$\beta$-actin & Sigma-Aldrich & A5441 \\
\hline GAPDH & EMD Millipore & CB1001 \\
\hline anti-rabbit-HRP & Jackson ImmunoResearch & $711-035-152$ \\
\hline anti-mouse-HRP & Jackson ImmunoResearch & $715-035-151$ \\
\hline
\end{tabular}

Supplementary Table 2. Oligonucleotides for real-time quantitative PCR analysis.

\begin{tabular}{|l|l|l|}
\hline Name & Forward (5' to $\mathbf{3}^{\prime}$ ) & Reverse (5' to $\mathbf{3}^{\prime}$ ) \\
\hline ALDH1A1 & AGTGTGGGTGATTGCTATGG & TGTGACTGTTTTACCTCTGT \\
\hline ALDH1A2 & AAGATGTCTGGAAATGGGAGAG & GGGATCTTTACTGTCACCGTC \\
\hline ALDH1A3 & CTTCTGCCTTAGAGTCTGGAAC & TGCTACGTGGACAAGAACTG \\
\hline ALDH1B1 & CCCAAGCGTGATCCTGAAC & ATGTCTGGGTTCAGAATGGG \\
\hline ALDH1L1 & GTACAACCGCTTCCTCTTCC & TTGATCTTGGCTGTCTCCTTC \\
\hline ALDH1L2 & ACGACACTATCAACCCAACAG & TCTTCCCCATTCACCGTTTTC \\
\hline ALDH2 & ATGAGTTTGTGGAGCGGAG & TTCCCCGTGTTGATGTAGC \\
\hline ALDH3A1 & CCTTAAATACGTCCCCTCTTGG & TCGCTGATCTTGCTCATGG \\
\hline ALDH3A2 & TGTTGCTCACTTTCCTGGG & GCTGGGTTTTGAATCTGGTG \\
\hline ALDH3B1 & AGCCATCGGAGATTAGCAAG & AAGATGTAGTCGAACCTGTGC \\
\hline ALDH3B2 & TTCCTTCAAGAAAACAAGCAGC & CAGGTTCTTGAGAGCGTAGTC \\
\hline ALDH4A1 & CCGCTTCTAACCCGAGATG & CCTGCGTGAAGGCTAAGAC \\
\hline ALDH5A1 & TGGCACCAGTTATCAAGTTCG & AACCATGCCCACTTCCAG \\
\hline ALDH6A1 & TGAAGGAGATGTATTTCGAGGC & GAGGCAGACGGTAGGAATAAAG \\
\hline ALDH7A1 & AGTATGCGTGGCTGAAAGAG & CCTGTCGGACTCTTGCTATTG \\
\hline ALDH8A1 & AAAGTCGGCATTCCCTCTG & CAACTTATCCACTCCCTCACC \\
\hline ALDH9A1 & ACAACTATAACGTCAGCCCAG & CACATCACCCATCTCCACAC \\
\hline ALDH16A1 & GCTGGAGCTGGGCTATG & GCCGCAGATACTCATACAGC \\
\hline ALDH18A1 & ATCTGGCTGATCTGTTGACG & GGCTGTTCAATTTGGATGTGG \\
\hline B-actin & ACCTTCTACAATGAGCTGCG & CCTGGATAGCAACGTACATGG \\
\hline GAPDH & CCCACTCCTCCACCTTTGAC & ATGAGGTCCACCACCCTGTT \\
\hline
\end{tabular}


Supplementary Table 3. siRNA and shRNA sequences.

\begin{tabular}{|l|l|l|l|}
\hline \multicolumn{1}{|c|}{ Gene } & \multicolumn{1}{|c|}{ Name } & \multicolumn{1}{|c|}{ Sequence } & \multicolumn{1}{c|}{ Dharmacon catalog \# } \\
\hline Control & siCtl & UGGUUUACAUGUCGACUAA & D-001810-01 \\
\hline SOX9 & siSOX9 & GAACGCACATCAAGACGGA & D-059108-01 \\
\hline SOX9 & sh-1 & TATACAGAAATTGAAGGATGC & RHS3979-201751501 \\
\hline SOX9 & sh-2 & TTCATGTAGGTGAAGGTGGAG & RHS3979-201751503 \\
\hline
\end{tabular}




\section{Chapter 3: SOX9 regulates cancer stem-like properties and metastatic potential of single-walled carbon nanotube-exposed cells}

Maria A. Voronkova", Sudjit Luanpitpong"§, Liying Wang Rojanasakul", Vincent Castranova $^{\ddagger}$, Cerasela Zoica Dinull, Heimo Riedel ${ }^{\wedge \#}$, and Yon Rojanasakul ${ }^{\star \wedge,} \neq$

West Virginia University Cancer Institute, ${ }^{\ddagger}$ Department of Pharmaceutical Sciences, "Department of Chemical Engineering, "Department of Biochemistry, West Virginia University, WV 26506, United States; ${ }^{\circledR}$ Siriraj Center of Excellence for Stem Cell Research, Faculty of Medicine Siriraj Hospital, Mahidol University, Bangkok 10700, Thailand; "Allergy and Clinical Immunology Branch, National Institute for Occupational Safety and Health, Morgantown, WV 26505, United States.

As published in:

Voronkova MA, Luanpitpong S, Rojanasakul LW, Castranova V, Dinu CZ, Riedel H, et al. SOX9 Regulates Cancer Stem-Like Properties and Metastatic Potential of SingleWalled Carbon Nanotube-Exposed Cells. Sci Rep. 2017;7(1):11653. 


\begin{abstract}
Engineered nanomaterials hold great promise for future development of innovative products, but their adverse health effects are a major concern. Recent studies have indicated that certain nanomaterials, including carbon nanotubes (CNTs), may be carcinogenic. However, the underlying mechanisms behind their potential malignant properties remain largely unknown. In this study, we investigated the potential role of SOX9, a stem cell associated transcription factor, in neoplastic transformation of human lung epithelial cells after chronic exposure to low-dose single-walled carbon nanotubes (SWCNTs). We found that SOX9 is upregulated in chronic SWCNT-exposed cells, which is consistent with their abilities to induce tumor formation and metastasis in vivo. We therefore hypothesized that SOX9 overexpression may be responsible for the neoplastic-like phenotype observed in our model. Indeed, SOX9 knockdown inhibited anchorage-independent cell growth in vitro and lung colonization in vivo in a mouse xenograft model. SOX9 depletion also suppressed the formation of cancer stem-like cells (CSCs), as determined by tumor sphere formation and aldehyde dehydrogenase (ALDH) activity (Aldefluor) assays. Furthermore, SOX9 knockdown suppressed tumor metastasis and the expression of the stem cell marker ALDH1A1. Taken together, our findings provide a mechanistic insight into SWCNT-induced carcinogenesis and the role of SOX9 in CSC regulation and metastasis.
\end{abstract}




\section{Introduction}

Engineered nanomaterials have increasingly been used for various applications, but their long-term health effects are largely unknown. Carbon nanotubes (CNTs) are one of the most commonly used engineered nanomaterials due to their unique properties such as light weight, high tensile strength, and electrical conductivity ${ }^{1,2}$. However, CNTs have some negative properties as well, such as a high aspect ratio and biopersistence; therefore, questions about their potential carcinogenicity have been raised $^{3,4}$. Previous animal studies have shown that pulmonary exposure to single-walled carbon nanotubes (SWCNTs) induces inflammation, granulomas, and fibrosis ${ }^{5,6}$, conditions that have been associated with an increased risk of lung cancer ${ }^{7,8}$. In fact, some CNTs can induce or promote tumor formation in animals ${ }^{3,9-12}$. Furthermore, one type of CNTs, multi-walled carbon nanotubes (MWCNTs) Mitsui-7, was classified as possibly carcinogenic to humans by the International Agency for Research on Cancer $(\mathrm{IARC})^{13}$, while in vitro data on other CNT types were concluded insufficient to be extrapolated to humans.

We previously reported that long-term, low-dose exposure of human lung epithelial cells to SWCNTs and MWCNTs results in neoplastic-like transformation ${ }^{14,15}$. Long-term treatment with CNTs was applied to mimic gradual cellular transformation during cancer development, a process that may require a prolonged exposure to carcinogens ${ }^{16-18}$. We also reported that chronically SWCNT-exposed cells contain a highly invasive and tumorigenic stem-like cell subpopulation ${ }^{19,20}$. However, detailed information about the underlying mechanisms remains unknown.

Increasing amounts of evidence suggest that cancer stem cells or stem-like cells (CSCs), also called tumor initiating cells, are the main driving force behind tumor formation and metastasis ${ }^{21,22}$. CSCs and regular stem cells share many properties, including self-renewal capacity, potency for differentiation, and resistance to apoptosis. More importantly, CSCs are typically resistant to chemotherapy and eventually give rise to recurrent tumors ${ }^{22,23}$.

Many stem cell regulatory proteins are now being recognized as oncogenes because of their ability to regulate CSCs. SOX9 (SRY (sex determining region Y)-box 9) 
is a member of the SOX family of transcription factors, which play critical roles in embryonic development, lineage commitment, and stem cell maintenance ${ }^{24}$. Notably, SOX9 is involved in lung branching morphogenesis ${ }^{25}$, and its expression is elevated in many types of cancer, including lung, skin, brain, and pancreatic cancers ${ }^{26}$. In non-small cell lung cancer (NSCLC), the most common type of lung cancer, SOX9 expression highly correlates with the disease progression and poor patient survival ${ }^{27,28}$. Accumulating evidence also suggests that SOX9 may regulate $\mathrm{CSCs}^{29-32}$. However, detailed mechanisms have yet to be elucidated. Furthermore, it is not known whether SOX9 plays a role in SWCNT-induced carcinogenesis and CSC formation.

In this study, we demonstrated that chronically SWCNT-exposed human lung cells display high levels of SOX9 expression and contain a distinct CSC subpopulation. We hypothesized that SOX9 overexpression may be responsible for the malignant phenotype observed in these cells. Consequently, we evaluated the effects of SOX9 expression on the tumorigenicity, invasiveness, and stemness of SWCNT-transformed cells in vitro and in vivo. 


\section{Results}

\section{Exposure to carbon nanotubes induces cell transformation}

To test the potential role of SOX9 in SWCNT-induced oncogenesis, human bronchial epithelial Beas-2B cells were continuously exposed to occupationally relevant concentrations $\left(0.02 \mu \mathrm{g} / \mathrm{cm}^{2}\right)$ of SWCNTs for a period of 6 months, as previously described ${ }^{14,15,19}$. Physicochemical properties of SWCNTs used in this study are summarized in Table 1. Potential carcinogenic properties and SOX9 expression were then evaluated in vitro and in vivo. Analysis of anchorage-independent cell growth, a hallmark of cancer ${ }^{33,34}$, revealed that the SWCNT-treated cells (termed BSW) formed 5fold more colonies when compared to passage-matched control (B2B) cells (Fig. 1a). To evaluate the tumorigenic potential of BSW cells in vivo, these cells and the control B2B cells were genetically labeled with luciferase and injected subcutaneously into the flanks of NOD/SCID gamma mice. Tumor formation was examined over a period of 4 weeks by external caliper measurements and by bioluminescence imaging. Unlike control cells, BSW cells formed rapidly growing tumors (Fig. 1b, c). Moreover, ex vivo analysis at the end of the experiments showed spontaneous metastasis of the BSW cells to the mouse lungs and liver (Fig. 1d, e, f and Supplementary Fig. S1). These results indicate that SWCNT-transformed cells possess tumorigenic and metastatic properties.

\section{SOX9 overexpression regulates malignant properties of BSW cells}

Cancer stem cells (CSCs) are considered to be the main source of cancer metastasis, chemoresistance, and tumor recurrence ${ }^{21}$. Previous studies have shown that SWCNT-exposed cells contain a CSC-like subpopulation identical to CSCs reported in lung cancer ${ }^{19,35}$. This cell population is characterized by the expression of stem cell markers, self-renewal ability, and more importantly by chemoresistance and high tumorigenic potential ${ }^{19,20}$. In this study, we examined the molecular mechanisms of CSC regulation to identify possible biomarkers and drug targets for CNT-related malignancies. SOX9, a stem cell transcription factor, has recently been implicated in CSC regulation ${ }^{26,29}$ and is overexpressed in non-small cell lung cancer (NSCLC) ${ }^{27,32}$. We hypothesized that SOX9 may regulate the tumorigenic and metastatic properties of 
SWCNT-exposed cells by controlling CSCs. Indeed, BSW cells express a high level of SOX9 protein when compared to passage-matched control cells (Fig. 2a). Furthermore, immunostaining analysis revealed that lungs of all mice in the BSW group contain SOX9 overexpressing micrometastases (Fig. 2b). Immunofluorescent staining for CSC markers in control and SWCNT-exposed cells demonstrated that CSC markers are overexpressed in BSW cells and that the CSCs are the cells that have elevated SOX9 expression (Supplementary Fig. S2).

To test our hypothesis, SOX9 expression in BSW cells was stably knocked down by shRNAs (Fig. 3a). The knockdown cells (shSOX9) exhibited a slower rate of proliferation (Fig. 3b) and formed substantially fewer colonies in the soft agar assay when compared to vector control cells (Fig. 3c, d). These results suggest that SOX9 depletion alleviates the survival of cells in matrix depleted conditions and potentially decreases their metastatic potential. In vitro migration and invasion assays further demonstrated a marked reduction in cell motility following SOX9 knockdown (Fig. 4). We also used an established NSCLC cell line H460 to compare the results of SOX9 downregulation in BSW cells to those in lung cancer cells. SOX9 knockdown also attenuated colony formation and decreased the proliferation rate of $\mathrm{H} 460$ cells (Supplementary Fig. S3), which is consistent with previous reports ${ }^{28,32}$. Taken together, our results support the critical role of SOX9 overexpression in the malignant phenotype of SWCNT-exposed cells. Specifically, SOX9 knockdown reduced cell proliferation, colony formation, migration, and invasion - properties that all are considered as hallmarks of cancer ${ }^{33}$.

\section{SOX9 regulates SWCNT-induced cancer stem cells}

The ability for self-renewal is a key characteristic of stem cells ${ }^{36,37}$. The tumor sphere formation assay has frequently been used as a functional in vitro test to evaluate the self-renewal ability of adult stem cells and cancer stem cells ${ }^{38,39}$. To evaluate the sphere forming capability of SWCNT-transformed cells and its regulation by SOX9, 1,000 control and shSOX9 cells were cultured under non-adherent conditions in serumfree medium. After 2 weeks in culture, the number of tumor spheres exceeding $50 \mu \mathrm{m}$ in 
diameter was quantified. We found that SOX9 knockdown substantially inhibited sphere formation (Fig. 5a), suggesting that SOX9 positively regulates stem cells in our model.

Next, we evaluated the expression and activity of the common cancer stem marker ALDH, which is highly active in cancer stem cells and is frequently associated with poor clinical outcomes ${ }^{23,40,41}$. The Aldefluor assay was used to quantify ALDH activity in BSW and BSW-shSOX9 cells. The activated Aldefluor reagent is a cellpermeable fluorescent substrate for ALDH that accumulates inside cells after interaction with ALDH and can be subsequently detected using flow cytometry. Diethylaminobenzaldehyde (DEAB), a specific ALDH inhibitor, was used to evaluate background fluorescence and set up gates for flow cytometry. We found that ALDH activity was substantially decreased in SOX9 knockdown cells, as indicated by a reduced number of cells exhibiting high ALDH activity (ALDH ${ }^{\text {hi }}$ cells) (Fig. 5b, c). Consistent with the pattern of ALDH activity, expression levels of ALDH1A1, one of the main CSC-associated ALDH isoforms ${ }^{42,43}$, were dramatically reduced following SOX9 knockdown (Fig. 5d). ALDH1A1 expression was also depleted in H460-shSOX9 cells (Supplementary Fig. S3), indicating that a correlation between SOX9 and ALDH expression is not limited to one specific cell system. These results suggest that SOX9 controls CSCs in BSW cells and possibly other cancer cell types through ALDH1A1.

\section{SOX9 knockdown attenuates BSW metastasis in vivo}

We next evaluated whether SOX9 depletion affects the metastatic potential of BSW cells in vivo. Luciferase-labeled BSW and BSW-shSOX9 cells were intravenously injected into mice followed by weekly bioluminescence imaging. At the end of the experiment, mice were euthanized; their internal organs (brain, liver, spleen, pancreas, kidneys and lungs) were removed and imaged to evaluate metastatic lesions. Figure $6 \mathrm{a}$ shows representative whole-body bioluminescence images of mice bearing either the control or shSOX9 cells. Importantly, lung colonization by shSOX9 cells was dramatically decreased when compared to control cells (Fig. 6b). This was also confirmed by ex vivo lung imaging (Fig. 6c) and by histological analysis (Fig. 6d). The luminescent signals from distant metastases in the liver and brain also decreased (Fig. $6 e, f)$. In addition, we observed occasional luciferase signals from the kidneys and 
pancreas in the control but not in the shSOX9 animals (data not shown). Our findings indicate a significant role of SOX9 in the regulation of the metastatic properties of malignantly transformed BSW cells.

\section{SOX9 overexpression in normal Beas-2B cells induces stem cell-like properties}

We next overexpressed SOX9 in passage-matched control (B2B) cells to test whether this would be sufficient to recapitulate the phenotype of BSW cells. Of note, we achieved a maximum 5 -fold increase of SOX9 expression (Fig. $7 \mathrm{a}$ ), while BSW cells have on average a 15-fold increase in SOX9 expression in comparison to Beas-2B cells (Fig. 2a). Nevertheless, a 5 -fold SOX9 overexpression in Beas-2B cells was sufficient to promote tumor sphere formation (Fig. $7 b, c)$. Note that these cells normally form a small number of loose cell aggregates under low-attachment conditions, while SOX9 overexpression triggered the appearance of typical, round tumor spheres. Furthermore, mRNA levels of main NSCLC-associated isoforms ALDH1A1 and ALDH1A3 ${ }^{44}$ as well as ALDH activity were upregulated by SOX9 (Fig. 7d, e). We also evaluated changes in the expression levels of stem cell related proteins in response to SOX9 overexpression. We observed an increased expression of CD133, a marker of lung CSCs ${ }^{45}$, and embryonic stem cell factors Nanog, SOX2 and Oct4, although the increase was not statistically significant in the case of Oct4 (Fig. 7f). Together, these results support the regulatory role of SOX9 in lung CSCs induction and ALDH1A1 expression. 


\section{Discussion}

In this study, we demonstrated that lung epithelial cells chronically exposed to SWCNTs may undergo malignant transformation and gain metastatic properties. These cells displayed traits typically characteristic of cancer cells, including anchorageindependent growth and in vivo tumor formation, supporting the previously published work $^{14,19}$. In addition, the metastatic potential of SWCNT-exposed cells was demonstrated for the first time. We observed that BSW cells can metastasize in both subcutaneous (Fig. 1) and tail vein mouse models (Fig. 6). We showed for the first time that SOX9 is a critical player in SWCNT-induced carcinogenesis, and that SOX9 depletion significantly reduces metastatic potential both in vitro (Fig. 4) and in vivo (Fig. $6)$.

Cancer stem cells (CSCs) have been considered the main source of cancer initiation, dissemination, and recurrence based on their ability for self-renewal, drugand apoptosis resistance. Furthermore, multiple studies have illustrated that tumor initiating cells share some characteristics with regular stem cells and express stem cell markers. Thus, it is not surprising that many proteins that are involved in embryonic development, such as the SOX family of proteins, appear to be CSC drivers. Our study demonstrated a high level of SOX9 expression in aggressive SWCNT-exposed cells, consistent with recent reports of SOX9 up-regulation in multiple cancer types ${ }^{26}$. However, the functional role of SOX9 in transformed cells required further investigation. We hypothesized that the elevated SOX9 expression in cancer cells and SWCNTexposed cells may induce CSC formation, which in turn could drive tumor formation and metastasis. Indeed, we found that SOX9 knockdown strongly inhibited tumor sphere formation and anchorage-independent growth (Fig. 5a, Fig. 3c, d). Bioluminescence signals from mouse lungs in the tail vein model clearly demonstrated that SOX9 knockdown decreases lung colonization (Fig. 6). Together, these results suggest that SOX9 regulates cancer cell survival, consistent with our previous work in lung cancer ${ }^{32}$.

We tested how SOX9 affects CSC markers to further evaluate our hypothesis. Elevated activity of ALDH, an enzyme with a variety of functions including detoxification, is associated with poor clinical outcomes due to metastasis and has been linked to 
$\operatorname{CSCs}^{23,40,41}$. We observed a substantial decrease in ALDH activity following SOX9 knockdown (Fig. 5d, c), suggesting that ALDH may be a potential downstream target of SOX9. Likewise, the expression of ALDH1A1, an ALDH isoform most commonly associated with the CSC activity ${ }^{23,40,42}$, was dramatically reduced in both BSW and H460 knockdown cells (Fig. 5d, Supplementary Fig. S3). Notably, ALDH serves not only as a stem cell marker, but also governs stem cell differentiation ${ }^{46,47}$ by producing retinoic $\mathrm{acid}^{48}$. To further explore the potential relationship between ALDH and SOX9, we overexpressed SOX9 in control Beas-2B cells. We observed an increase in tumor sphere formation (Fig. 7b, c), which is consistent with the knockdown experiments. Likewise, mRNA levels of the reported to be overexpressed in NSCLC ALDH isoforms ALDH1A1 and ALDH1A3 ${ }^{44}$ were elevated, validating our findings in SOX9 knockdown cells. We also observed an increased expression of stem cell makers CD133, Nanog and SOX2 (Fig. 7 f), suggesting that SOX9 overexpression renders cells less differentiated. Collectively, our observations strongly support the positive regulatory role of SOX9 in CSC formation, consistent with other studies in pancreatic, esophageal, and colorectal cancers ${ }^{30,49,50}$. To our knowledge, the present study demonstrates the mechanistic link between SOX9 and ALDH expression for the first time. This provides a key mechanism underlying CSC induction by SOX9, while the exact mode of ALDH upregulation remains a subject for further investigation.

A limitation of this study is that the results were largely derived from one cell model (Beas-2B cells) and one type of CNTs. However, our group observed a similar neoplastic-like transformation in several other models and with different types of CNTs. For examples, MWCNTs have recently been shown to induce neoplastic transformation of primary human small airway epithelial cells ${ }^{51}$. Both SWCNTs and MWCNTs similarly induced such transformation in hTERT-immortalized human small airway epithelial cells after a long-term exposure ${ }^{15}$. The induction of fibroblast stem-like cells and ALDH1A1 expression was also reported in primary human lung fibroblasts exposed to SWCNTs or MWCNTs $^{51}$. Furthermore, co-culture of these activated fibroblasts with lung cancer cells promoted CSC-related properties and tumor formation by cancer cells ${ }^{52}$. Of note, animal experiments in the current study were conducted in immunodeficient mice, and therefore these results should be interpreted with caution. Nonetheless, several other 
studies using immunocompetent mice and rats consistently indicate the tumorigenic potential of SWCNTs and MWCNTs ${ }^{9-12}$. While SOX9 is implicated as a key regulator of SWCNT-induced carcinogenesis in this report, other regulatory mechanisms are likely to be involved due to the complexity of carcinogenic process. For example, Shvedova et al. showed that inhalation or aspiration of SWCNTs caused lung inflammation and fibrosis along with K-ras mutation in immunocompetent mice ${ }^{53}$. Since K-ras is a known oncogene, whose mutational activation is frequently associated with an increased risk of lung cancer, it is quite possible that such mutation may be involved in SWCNT-induced carcinogenesis, although this has not been demonstrated yet. In addition, several studies evaluating CNT carcinogenicity via whole transcriptome arrays have identified cancer signatures and known cancer prognostic markers in exposed mouse lung tissues $^{54,55}$. Some of the identified genes such as caveolin-1 and Bcl-2 have already been implicated in CNT-induced carcinogenesis ${ }^{20,56}$ and may be responsible for the early neoplastic transformation induced by SWCNTs.

Lung cancer is a progressive disease commonly associated with a long-term exposure to carcinogens. However, prolonged exposure studies to nanomaterials are lacking and are greatly needed for risk assessment and for safe-by-design strategies. Our results suggest that long-term exposure to SWCNTs could transform normal epithelial cells into metastatic tumor cells. Although multiple mechanisms and signaling pathways are likely to be involved in the transformation process, we report here the critical role of SOX9 in the transformation through CSC-related mechanisms. Given that CSCs may be involved in cancer initiation steps, we suggest that SOX9 may be used as an early biomarker for SWCNT-induced carcinogenesis. It remains to be elucidated whether SOX9 up-regulation and its contribution to malignancies are specific to SWCNTs or could be extrapolated to other CNTs. Such information will aid in the design of nanomaterial-specific biomarkers for risk assessment and safe-by-design efforts. 


\section{Methods}

Cell culture and exposure to SWCNTs. Immortalized human bronchial epithelial Beas-2B cells were obtained from the American Type Culture Collection (ATCC). Cells were cultured in advanced Dulbecco's modified Eagle medium (DMEM) (Life Technologies) supplemented with $1 \%$ fetal bovine serum (FBS, Atlanta Biologicals), $2 \mathrm{mM}$ L-glutamine, $20 \mathrm{mM} \mathrm{HEPES,} 100 \mathrm{units} / \mathrm{mL}$ penicillin and $100 \mu \mathrm{g} / \mathrm{mL}$ streptomycin in $5 \% \mathrm{CO}_{2}$ at $37{ }^{\circ} \mathrm{C}$. This cell model has been reported to be an appropriate model for in vitro lung carcinogenesis studies ${ }^{57}$. The cells were exposed to well-characterized single-walled CNTs (SWCNTs), as previously described ${ }^{14,19}$. Briefly, SWCNTs (Carbon Nanotechnology $(\mathrm{CNI})$ ) were purified by acid treatment to remove metal contaminates. Particle characterization studies were performed at $\mathrm{NIOSH}$ research facilities, and the results are summarized in Table 1. Particles were treated with acetone and placed in an ultrasonic bath at room temperature for $24 \mathrm{~h}$. The dispersed CNTs were then filtered from the solution using a $20 \mu \mathrm{m}$ nylon mesh screen followed by a $0.2 \mu \mathrm{m}$ polytetrafluoroethylene (PTFE) filter. After filter collection, the dispersed CNTs were washed thoroughly with distilled water to remove acetone. The filter was dried overnight in vacuum and weighed to determine the quantity of SWCNTs. The particles were suspended in phosphate-buffered saline (PBS) by brief sonication and added to the cells every 3-4 days when changing the culture medium. Subconfluent cultures of Beas-2B cells were continuously exposed to a low-dose, occupationally relevant concentration $\left(0.02 \mu \mathrm{g} / \mathrm{cm}^{2}\right.$ or $\left.0.1 \mu \mathrm{g} / \mathrm{mL}\right)$ of SWCNTs in culture for 6 months and passaged weekly. Cells were rinsed with PBS prior to culture medium changes and cell passaging to reduce potential SWCNT bioaccumulation over the exposure period.

The dose of SWCNTs used in this study was calculated based on reported effects of in vivo MWCNT exposure, normalized to mouse alveolar surface area. The lowest dose, which induced a biological response in vivo, was $10 \mu \mathrm{g} / \mathrm{mouse}$ lung $(0.5$ $\mathrm{mg} / \mathrm{kg}$ body weight $)^{6}$. Dividing this dose by the average mouse alveolar surface area $\left(\sim 500 \mathrm{~cm}^{2}\right)$ gives the in vitro surface area dose of $0.02 \mu \mathrm{g} / \mathrm{cm}^{2}$, which is roughly equivalent to a human lung burden for 8 hours/day over a month at $400 \mu \mathrm{g} / \mathrm{m}^{3}$ (high CNT level reported in a research facility) ${ }^{58}$ or about 3 years at $10 \mu \mathrm{g} / \mathrm{m}^{3}$ (average CNT 
level in U.S. facilities ${ }^{59}$. The cells were cultured in normal medium without SWCNTs for at least ten passages prior to further experiments Control cells were cultured for the same period of time in the above described culture medium. $\mathrm{H} 460(\mathrm{NCl}-\mathrm{H} 460)$ cells were purchased from the ATCC and were passaged less than 20 times. H460 cells were maintained in RPMI medium (Corning) supplemented with 10\% FBS, 100 units $/ \mathrm{mL}$ penicillin, and $100 \mu \mathrm{g} / \mathrm{mL}$ streptomycin.

Plasmids and generation of stable cell lines. pLKO.1 lentiviral plasmids for SOX9 knockdown generation were purchased from GE Healthcare (\#RHS4533EG6662). An empty vector was used as a control, and the virus was produced in HEK293T cells (ATCC). Briefly, HEK293T cells were co-transfected with the shRNA containing pLKO.1 plasmids or empty pLKO.1 vector, pCMV-VSV-G envelope, and psPAX2 packaging plasmids (Addgene \#8454, 12260) in the presence of FuGene 6 transfection reagent (Promega). The medium containing viral particles was collected at 24, 36, and 48 hours post-transfection, pooled, and used to infect SWCNT-exposed (BSW) cells in the presence of hexadimethrine bromide (Sigma-Aldrich) at a final concentration of $5 \mu \mathrm{g} / \mathrm{mL}$. After infection, cells were selected with $1 \mu \mathrm{g} / \mathrm{mL}$ of puromycin (Life Technologies). For SOX9 overexpression, SOX9 cDNA from pCMV-AC-GFPSOX9 (Origene) was amplified by PCR introducing BamHI/Sall restriction sites and was subcloned into pLenti CMV GFP Zeo (Addgene \#17449), replacing GFP. pLenti CMV GFP Zeo vector was used as a control. For flow cytometry experiments (Aldefluor assay), control vector with RFP instead of GFP was used. Viral particles were produced as described above.

Immunoblotting. Cells were lysed in a non-reducing loading buffer containing 63 $\mathrm{mM}$ Tris- $\mathrm{HCl} \mathrm{pH} 6.8,10 \%$ glycerol, and $2 \%$ sodium dodecyl sulfate (SDS). Protein concentrations were quantified using the Pierce BCA protein assay kit (ThermoScientific). Proteins $(40 \mu \mathrm{g})$ were resolved under denaturing conditions by $7.5-12 \%$ SDS-polyacrylamide gel electrophoresis (SDS-PAGE) and transferred onto PVDF membranes (EMD Millipore). Membranes were blocked in 5\% non-fat milk in TBS-T buffer (25 mM Tris-HCl, pH 7.4, $125 \mathrm{mM} \mathrm{NaCl}$, and 0.1\% Tween 20) for 1 hour, washed 3 times for 5 minutes with TBS-T buffer, and incubated with appropriate primary antibodies at $4 \stackrel{\circ}{\circ} \mathrm{C}$ overnight. Membranes were rinsed 3 times for 5 minutes with TBST-T 
buffer; membranes were then incubated with appropriate HRP-conjugated secondary antibodies (Jackson Immunoresearch), diluted in TBS-T for 1 hour at room temperature. Immune complexes were detected by enhanced chemiluminescence (Pierce ECL, Thermo-Scientific; Immobilon HRP Substrate, Millipore) and quantified with the Image Studio Lite program (Li-COR Biosciences). Antibodies used in this study are listed in the Supplementary Table 1. Full length unprocessed blot images are provided in the Supplementary Materials.

Flow cytometry. The ALDH hi population (cells with high aldehyde dehydrogenase activity) was identified by staining cells with the Aldefluor kit (Stem Cell Technologies), according to the manufacturer's instructions. Briefly, for each sample, $3 \times 10^{5}$ cells were incubated in the Aldefluor assay buffer with the activated Aldefluor substrate for 45 minutes at $37^{\circ} \mathrm{C}$ in the presence or absence of the specific ALDH inhibitor diethylaminobenzaldehyde (DEAB). Tubes stained with both the Aldefluor substrate and DEAB served as a negative control for each sample. ALDH converts Aldefluor substrate into a fluorescent product, and cells with high ALDH activity (ALDH ${ }^{\text {hi }}$ ) were detected by BD Fortessa cell analyzer (BD Biosciences). The flow cytometry gates were set to obtain $0.1 \% A L D H^{\text {hi }}$ cells in substrate + inhibitor tubes for each sample. All experiments were performed at least 3 times.

Soft agar colony formation assay. Cells were suspended in a soft agar medium containing $0.33 \%$ Difco agar (BD Biosciences) in 2x EMEM medium (Lonza) supplemented with L-glutamine, antibiotics and $15 \% \mathrm{FBS}$, and plated onto 6 -well plates over a layer of $0.5 \%$ soft agar medium. Cells were cultured under standard cell culture conditions and were fed with $0.2 \mathrm{~mL}$ of the regular medium twice a week. Plates were observed under a light microscope after 2 weeks, and colonies exceeding $50 \mu \mathrm{m}$ in diameter were scored. Experiments were performed in triplicates; at least 5 fields of view for each replicate were evaluated.

Migration and invasion assays. Cells were seeded in the upper chamber of Transwell matrigel coated (invasion) or control inserts (migration) with $8 \mu \mathrm{m}$ pores (Corning) in serum-free medium and were allowed to migrate or invade toward the serum-containing bottom chamber for 15 hours. Cells were subsequently removed from the top part of the chamber using a cotton swab, and cells on the bottom side were 
stained with crystal violet. Experiments were performed in at least duplicates, 5 random fields were photographed under a light microscope for each replicate.

Cell proliferation assay. The rate of cell proliferation was measured by seeding equal numbers of cells onto 6-well plates in duplicates and counting cells with the Countess automated cell counter (Life Technologies) every 2 days until plates become confluent.

Tumor sphere formation assay. Cells were plated onto ultra-low attachment 24-well plates (Corning) in $0.8 \%$ methylcellulose (MC)-based serum-free medium (Stem Cell Technologies, \#H4100) supplemented with $20 \mathrm{ng} / \mathrm{mL}$ epidermal growth factor (BD Biosciences), $10 \mathrm{ng} / \mathrm{mL}$ basic fibroblast growth factor (Sigma-Aldrich), and $5 \mathrm{mg} / \mathrm{mL}$ insulin (Sigma-Aldrich). The number of tumor spheres exceeding $50 \mu \mathrm{m}$ in diameter (20 $\mu \mathrm{m}$ for Beas-2B cells) was quantified under a light microscope after 2 weeks of culture under standard cell culture conditions. All experiments were performed in triplicates and repeated at least twice, with 5 fields of view analyzed for each replicate.

Mouse xenograft experiments. All experiments were performed in accordance with the Guidelines for Animal Experiments at West Virginia University and approved by the Institutional Animal Care and Use Committee. Immunodeficient NOD/SCID gamma mice (NSG), strain NOD.Cg-Prkdc ${ }^{\text {scid }} \| 2 \mathrm{rg}^{\mathrm{tm} 1 \mathrm{~W}_{\mathrm{j}} /} / \mathrm{SzJ}$ (Jackson Laboratory), were injected with $1 \times 10^{6}$ of luciferase-labeled cells subcutaneously or via the tail vein. Tumor growth was monitored weekly using IVIS Lumina II in Vivo Imaging system (PerkinElmer), and an external caliper (VWR International) was used for the subcutaneous model. Tumor volume was calculated using the formula: tumor volume $\left[\mathrm{mm}^{3}\right]=1 / 2$ (length $\left.[\mathrm{mm}]\right) \times$ (width $[\mathrm{mm}]^{2}$ ). At the end of the experiments mice were euthanized, and their organs were removed and imaged ex vivo on a Petri dish to evaluate metastasis. Bioluminescent images were quantified using the Living Image software (PerkinElmer). Tissue processing and H\&E staining was carried out by the WVU Pathology Laboratory for Translational Medicine using standard procedures.

Immunohistochemistry. Tissue sections were deparaffinized using standard techniques. Antigen retrieval was carried out by heating slides in a microwave in citrate buffer (10 mM citric acid, 0.05\% Tween 20, pH 6.0) for $30 \mathrm{~min}$. Samples were blocked for 30 min with normal goat serum (BioGenex, \#HK112-9K) and incubated with primary 
antibodies overnight at $4{ }^{\circ} \mathrm{C}$ (specific antibodies used are listed in Supplementary Table 1). Slides were rinsed with TBS-T buffer 3 times and incubated with fluorescently labeled secondary antibodies (Life Technologies) for 1 hour at room temperature; after which they were rinsed with TBS-T buffer 3 times and mounted with the ProLong DAPI (Life Technologies). Cells were visualized with a Zeiss fluorescent microscope (Carl Zeiss).

Immunofluorescence. Cells were grown overnight on 8-well chamber slides (Nunc), fixed with $4 \%$ paraformaldehyde and permeabilized with $100 \%$ methanol at -20 ${ }^{\circ} \mathrm{C}$ prior to blocking with $5 \% \mathrm{BSA}$ in PBS. Cell were incubated with specific primary antibodies (listed in Supplementary Table 1), rinsed with PBS and incubated with secondary antibodies. Secondary antibodies were goat-anti-rabbit Alexa Fluor 546 (Life Technologies) and donkey-anti-mouse Alexa Fluor 488 (Jackson Immunoresearch). Slides were mounted with Fluoroshield with DAPI (Abcam) and visualized with a Zeiss fluorescent microscope (Carl Zeiss).

RT-qPCR. Total RNA was purified using the RNeasy kit (Qiagen). Reverse transcription was performed with SuperScript III Reverse Transcriptase (Life Technologies) using oligo(dT) primers. Quantitative real-time PCR was carried out using the SYBR Green Master Mix (Applied Biosystems) in an ABI 7500 Real-Time PCR Cycler (Applied Biosystems). Results were calculated using the $2^{-\Delta \Delta C t}$ method; glyceraldehyde-3-phosphate dehydrogenase (GAPDH) served as the internal control. Primer sequences are listed in the Supplementary Table 2.

Statistical analysis. The data represent means \pm SEM from two or more independent experiments as indicated. Statistical comparisons were made using twotailed Student's t test. When more than two groups were analyzed, ANOVA was used. Statistical analysis was performed with GraphPad Prism software (GraphPad), and $p<0.05$ was considered statistically significant.

Data Availability. The datasets generated during and/or analyzed during the current study are available from the corresponding author on reasonable request. 
Figures and legends

a
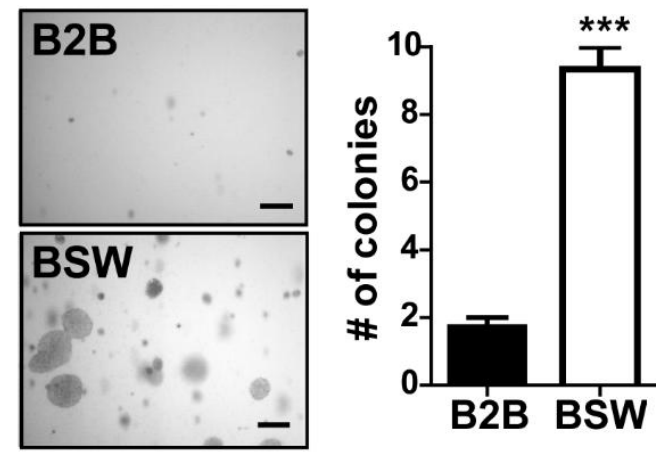

C

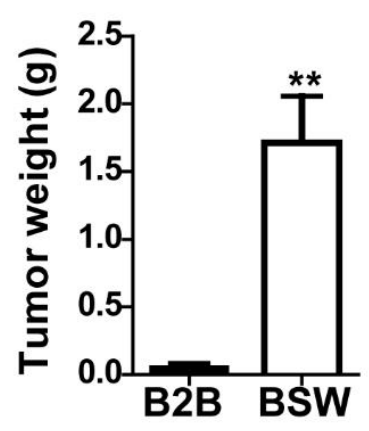

e

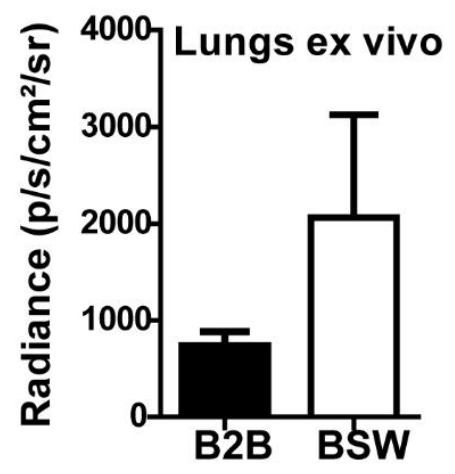

b

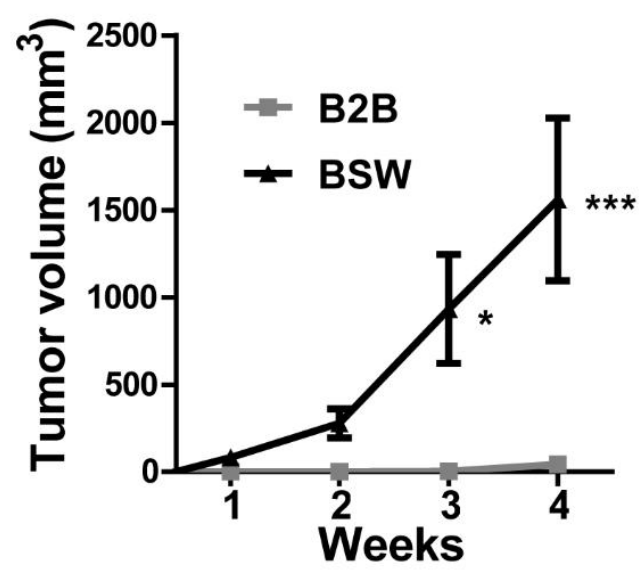

d B2B BSW

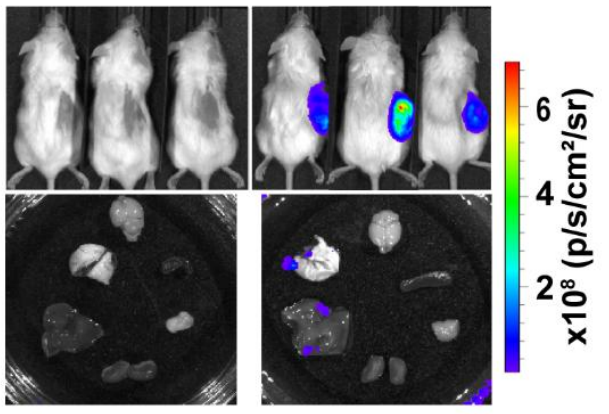

f

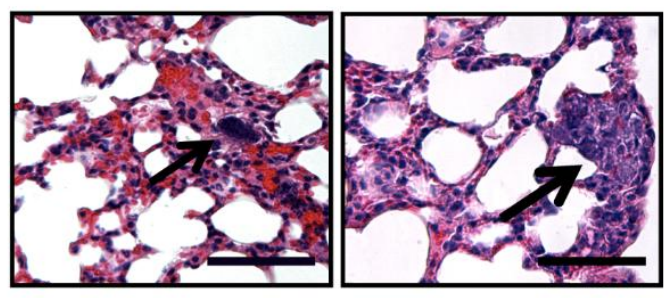


Figure 1. Human bronchial epithelial cells, chronically exposed to carbon nanotubes, undergo malignant transformation. (a) Chronic SWCNT-exposed bronchial epithelial Beas-2B (BSW) cells were cultured in soft agar for 2 weeks, and colonies over $50 \mu \mathrm{m}$ in diameter were counted, $\mathrm{n}=15, \mathrm{p}<0.0001$, two-tailed t-test. Scale bar $=500 \mu \mathrm{m}$. (b) Luciferase-labeled cells were injected subcutaneously into flanks of NOD/SCID gamma mice ( $n=5$ per group), and tumor volume was measured weekly by an external caliper. Means are different according to Bonferroni post hoc analysis following 2-way ANOVA, $p<0.05$. (c) Weight of isolated subcutaneous tumors at week $4, p=0.0014$, two-tailed t-test. (d) Top - representative bioluminescent signal from tumors at week 4, Bottom - representative luciferase signal from isolated internal organs. (e) Luciferase signal from lungs imaged ex vivo. (f) Representative images of H\&E stained lungs from mice bearing BSW cells. Arrows point to metastatic modules. Scale bar, $50 \mu \mathrm{m}$. Data are mean \pm SEM. 
a

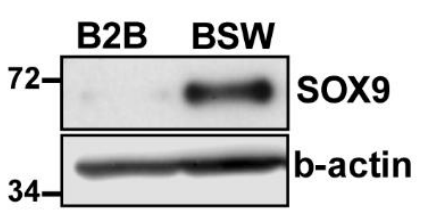

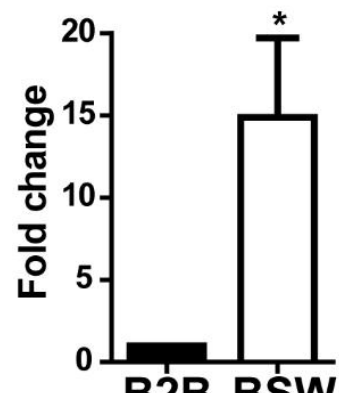

B2B BŚW b
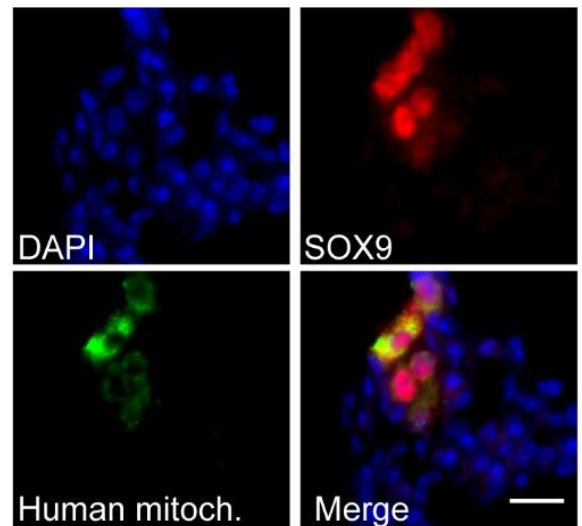

Figure 2. Human bronchial epithelial cells, chronically exposed to carbon nanotubes, overexpress SOX9. (a) Immunoblot of BSW and control cells, showing significant SOX9 overexpression in BSW cells. Right - relative SOX9 expression presented as fold change over control cells $(n=6)$. Data are mean $\pm S E M,{ }^{*}-p=$ 0.0347, paired two-tailed t-test. (b) Representative image of lung metastases from mice bearing BSW cells subcutaneously; sections were stained with anti-SOX9 antibody and antibody against human mitochondria, nuclei stained with DAPI. Scale bar: $20 \mu \mathrm{m}$. 

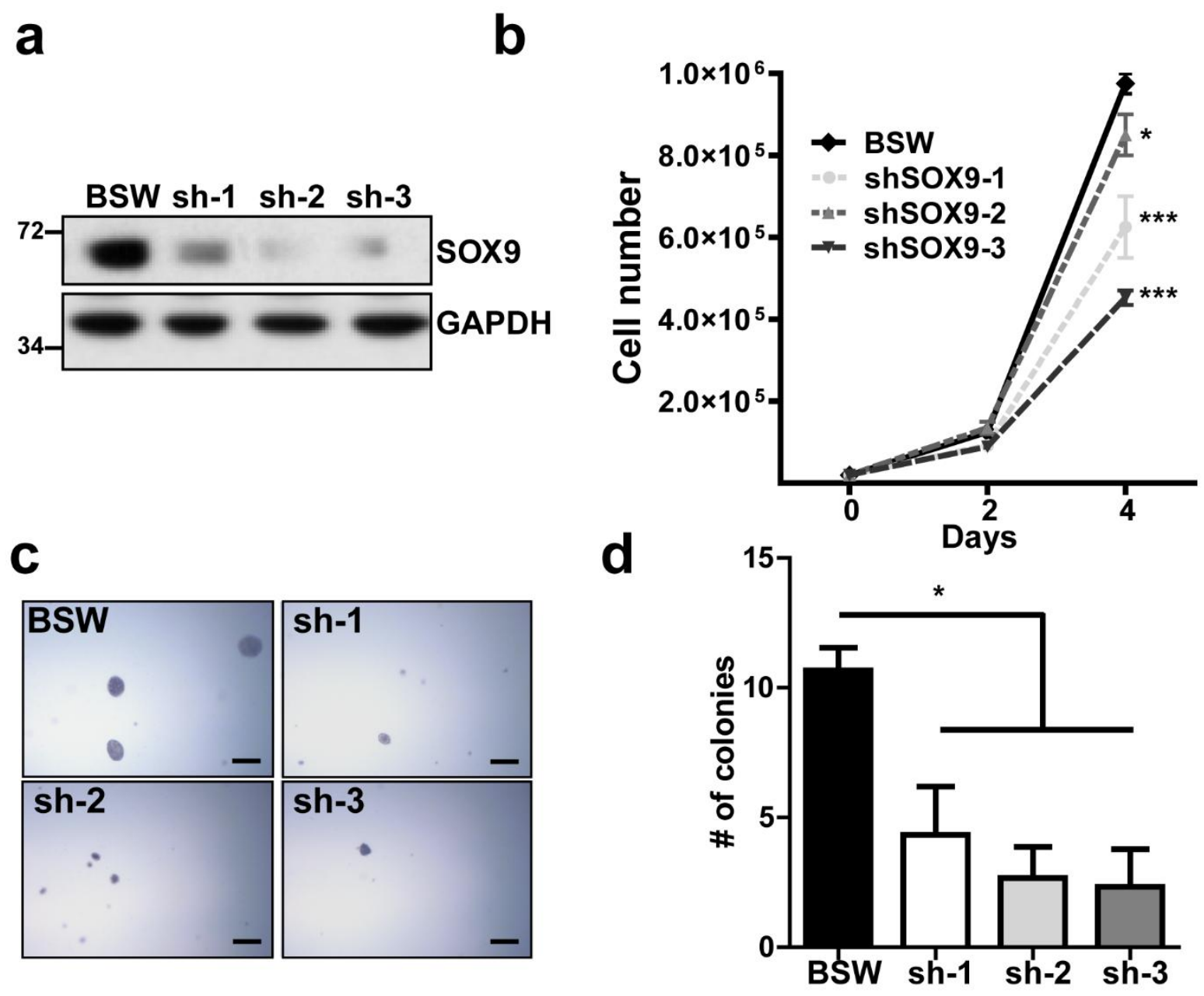

Figure 3. SOX9 knockdown inhibits proliferative and colony-forming properties of BSW cells. (a) Immunoblot showing levels of SOX9 protein after knockdown generated by 3 different shRNAs (sh-1, sh-2, sh-3), empty vector was used as a control in BSW cells. (b) Rate of cell proliferation, $p<0.05$, Bonferroni post hoc analysis following 2way ANOVA. (c) Soft agar colony formation assay. Cells were cultured in soft agar for 2 weeks, scale bar - $500 \mu \mathrm{m}$. (d) Quantification of the soft agar assay, colonies larger than $50 \mu \mathrm{m}$ in diameter were counted, $\mathrm{n}=3$. Data presented as mean values \pm SEM. Means are different according to Turkey post hoc analysis following ANOVA, $p<0.05$. 

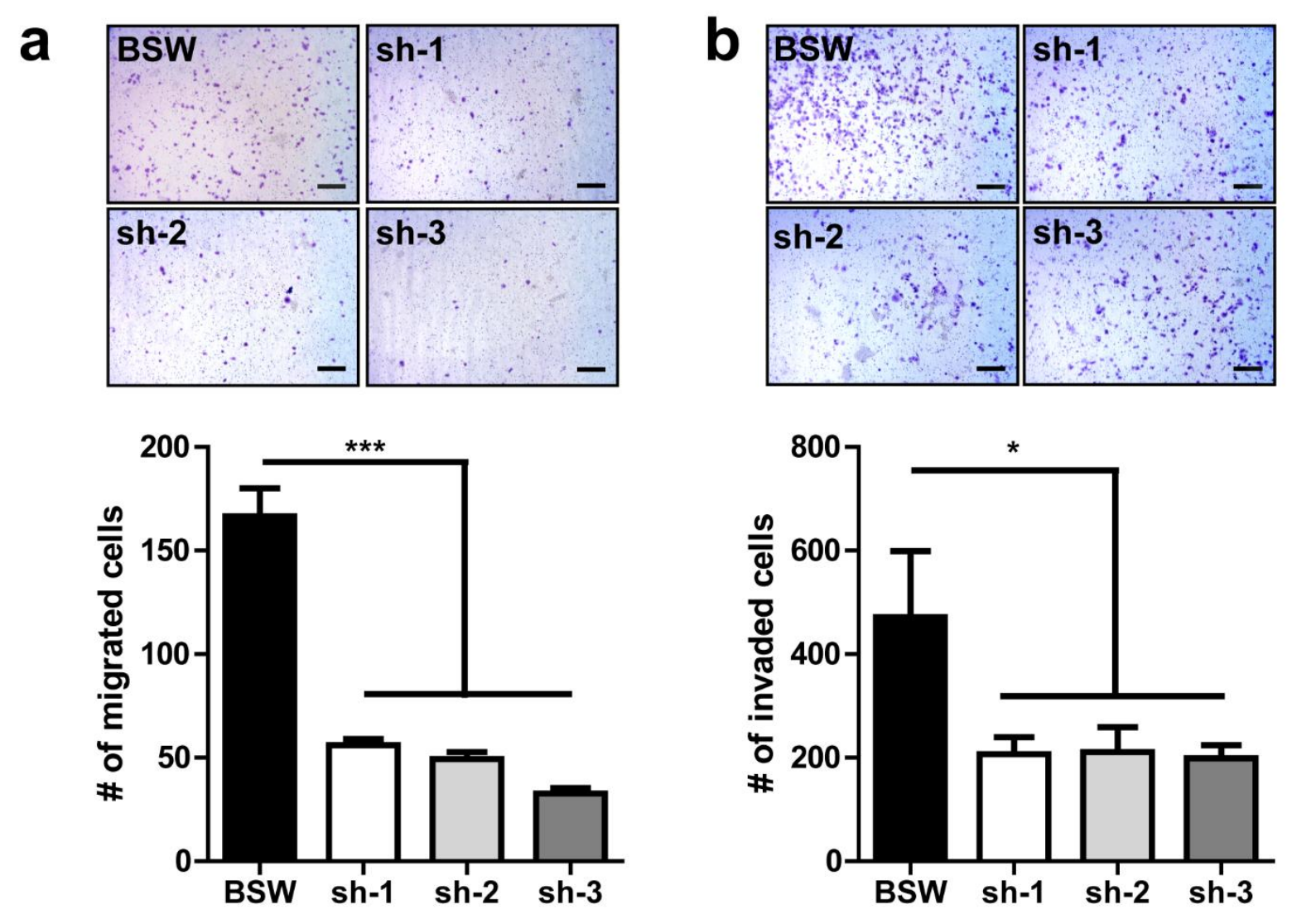

Figure 4. SOX9 knockdown attenuates migration and invasion of BSW cells in vitro. SOX9 knockdown was generated by 3 different shRNAs (sh-1, sh-2, sh-3), empty vector used as a control in BSW cells. BSW cells were added to control inserts (migration) or inserts coated with Matrigel (invasion) and incubated for $15 \mathrm{~h}$. After removal of non-migrated and non-invaded cells, cells were fixed and stained with crystal violet and counted under a microscope. (a) Transwell migration assay. Bottom quantification. (b) Transwell invasion assay. Bottom - quantification. Data presented as mean \pm SEM, $n=10$. Means are different according to Turkey post hoc analysis following ANOVA, $p<0.05$. 
a

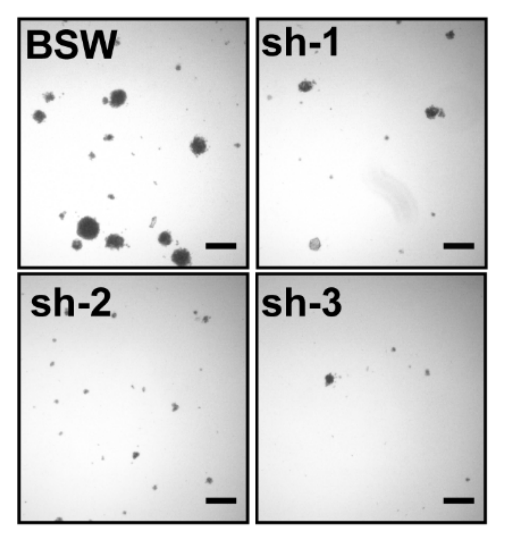

b

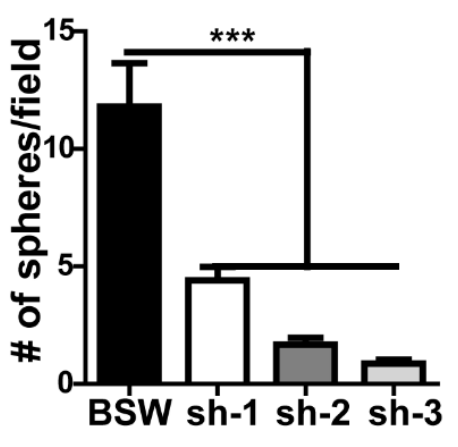

C
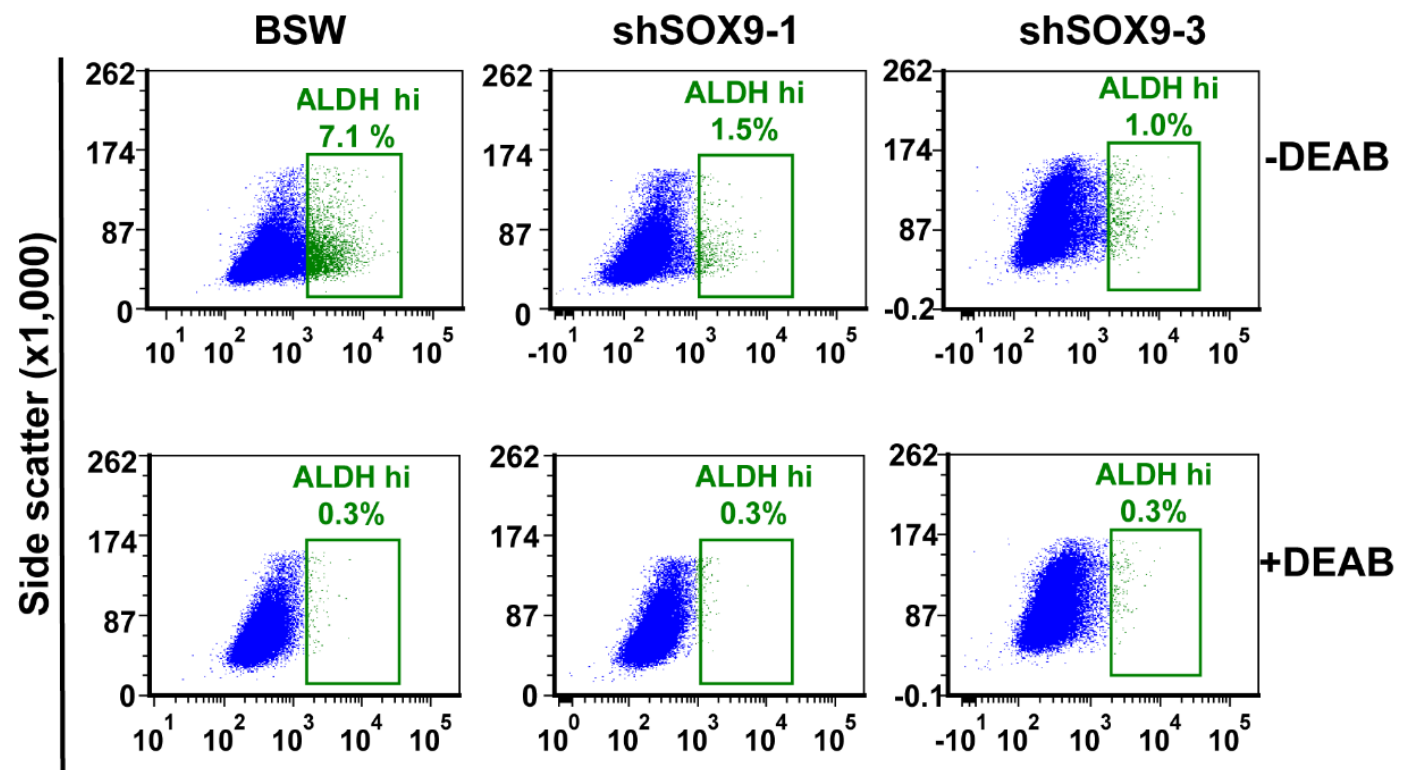

\section{Aldefluor}

d

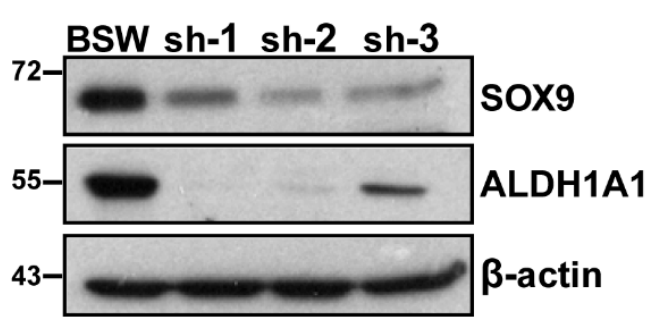

ALDH1A1 expression

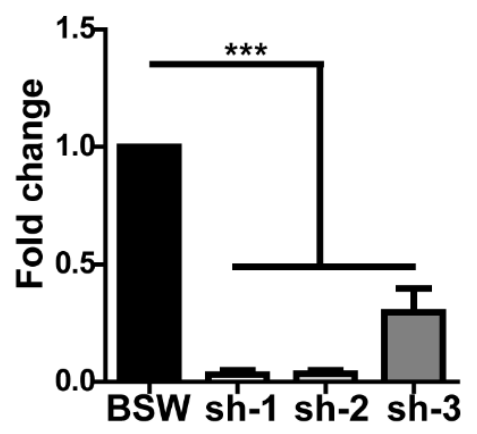


Figure 5. SOX9 regulates cancer stem cells in SWCNT-exposed cells. SOX9 knockdown was generated by 3 different shRNAs (sh-1, sh-2, sh-3), empty vector used as a control in BSW cells. (a) Tumor sphere formation assay. Right - quantitative analysis of tumor sphere formation, $n=15$. (b) Fold change in the number of $A L D H^{\text {hi }}$ cells in SOX9 knockdown over control BSW cells, 4 independent experiments combined ( $n=4$ - 9) measured by Aldefluor assay. (c) Representative flow cytometry results for Aldefluor assay. Top row - cells stained with the Aldefluor substrate only, bottom - cells stained with the Aldefluor substrate in the presence of the specific ALDH inhibitor DEAB. (d) SOX9 knockdown leads to a depletion of cancer stem cell marker ALDH1A1, right - quantification of relative ALDH1A1 expression, fold changes over control BSW cells, $n=3$ independent experiments. Results are mean \pm SEM. Means are different according to Turkey post hoc analysis following ANOVA, $p<0.05$. DEAB $-\mathrm{N}, \mathrm{N}-$ diethylaminobenzaldehyde. 

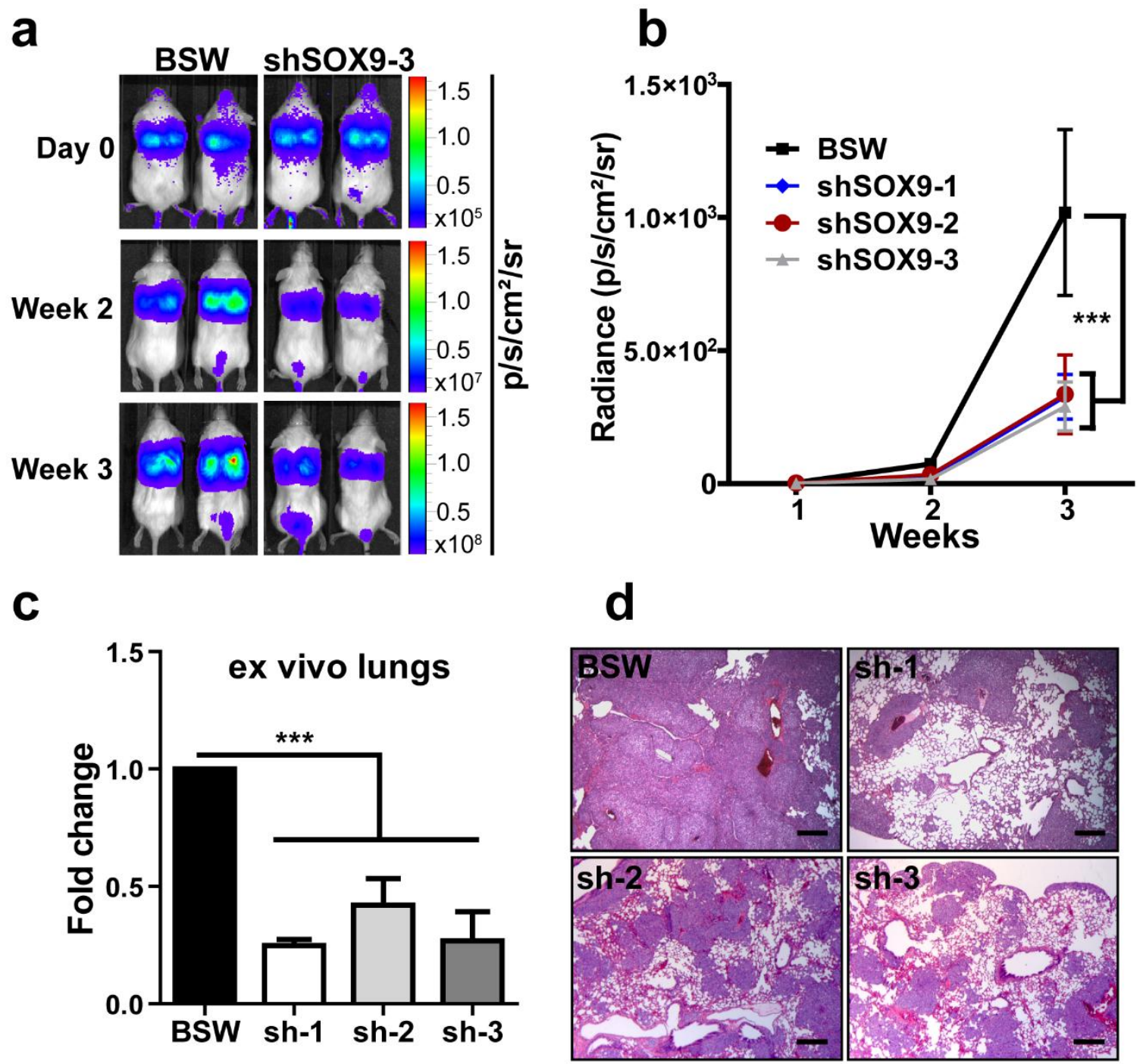

e

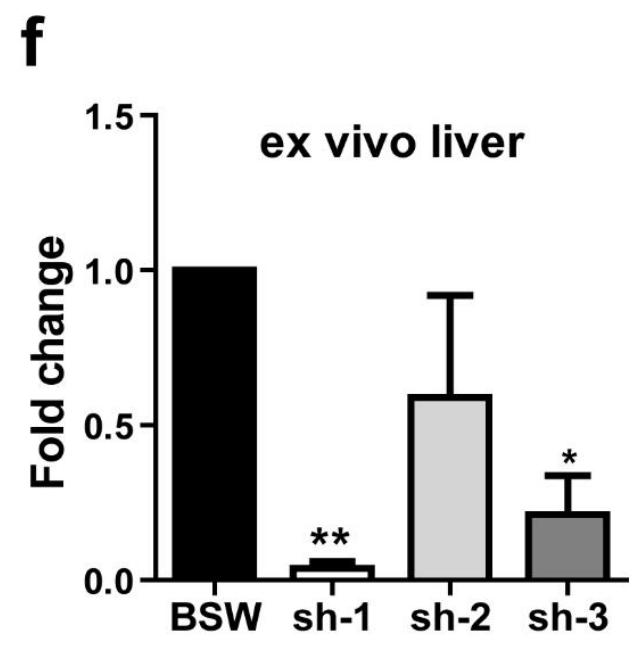


Figure 6. SOX9 knockdown inhibits BSW metastasis in vivo. Luciferase-labeled cells were injected intravenously via tail vein into NOD/SCID gamma mice ( $n=4$ per group) and mice were imaged right after cell injection and weekly thereafter. SOX9 knockdown was generated by 3 different shRNAs (sh-1, sh-2, sh-3), empty vector used as a control in BSW cells. (a) Representative whole-body bioluminescence of lung tumors in mice injected with control vector (BSW) or SOX9 knockdown (shSOX9-3). (b) Time course of lung tumor growth generated by weekly whole-body imaging, each time point normalized to the signal from day 0 . Means are different according to Bonferroni post hoc analysis following 2-way ANOVA, $\mathrm{p}<0.05$. (c, e, f) Ex vivo luciferase signal from isolated mouse lungs, brain and liver, respectively. Means are different according to Turkey post hoc analysis following ANOVA. (d) Representative pictures of H\&E stained lungs showing a notable decrease in tumor lung colonization by SOX9 knockdown cells comparing to control cells, scale bar - $250 \mu \mathrm{m}$. Data are shown as mean \pm SEM, $n=4$. 
a

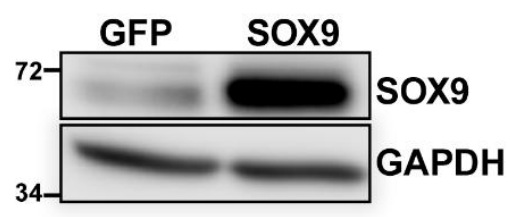

C

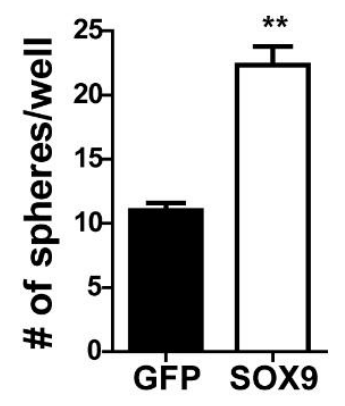

e

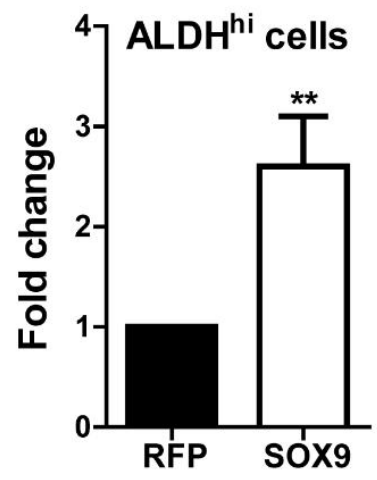

f b

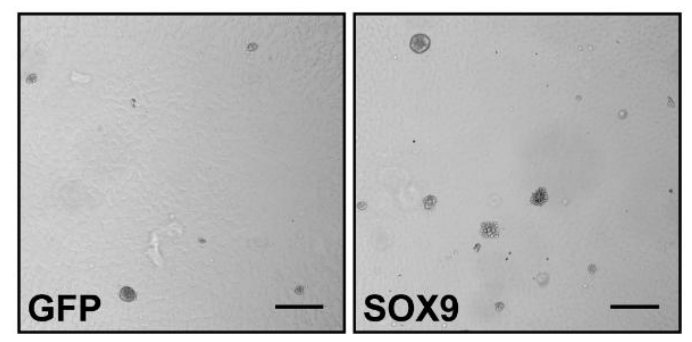

d
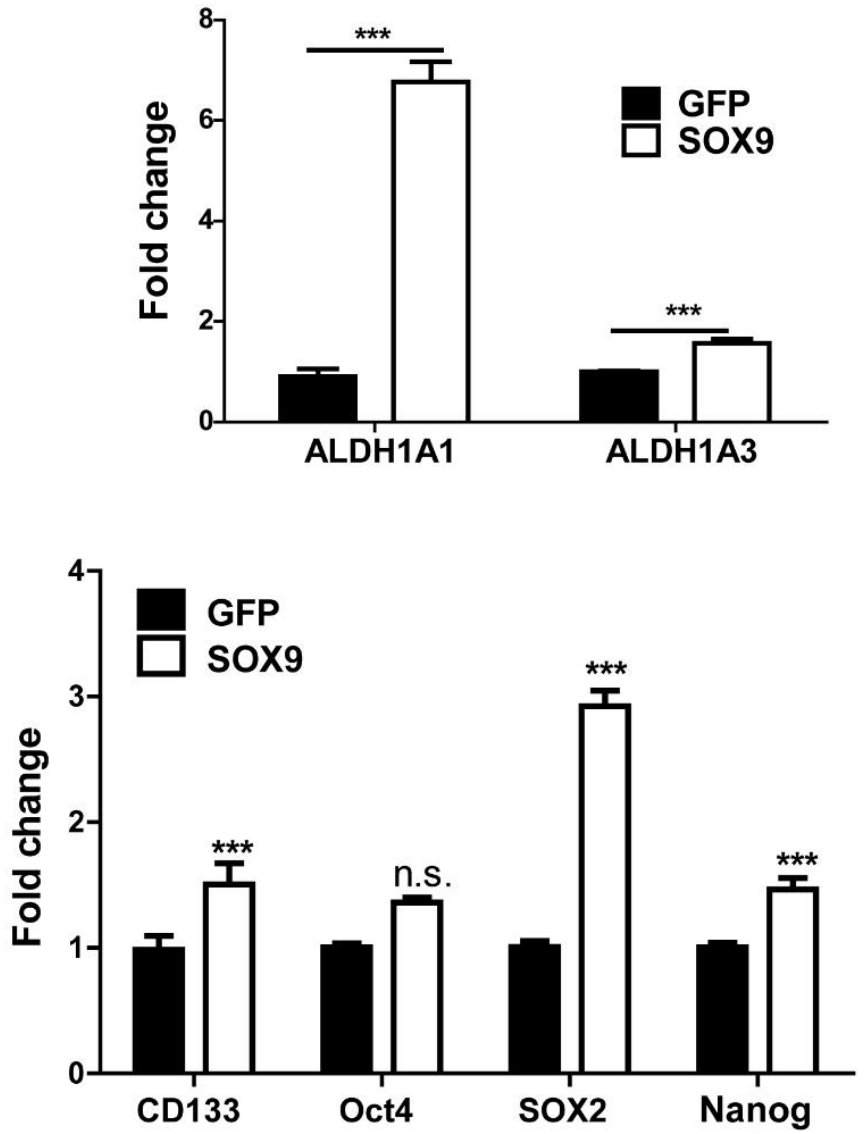
Figure 7. SOX9 overexpression in Beas-2B cells induces stem cell-like properties. (a) Level of SOX9 overexpression. (b) Representative images of tumor sphere formation. Scale bar, $200 \mu \mathrm{m}$. (c) Quantitative analysis of tumor sphere formation form (b), $n=3, p=0.0019$, two-tailed unpaired t-test. (d) RT-qPCR analysis of $2 \mathrm{ALDH}$ isoforms, $\mathrm{n}=6-12,2$ independent experiments combined, $\mathrm{p}<0.05$ by two-tailed unpaired t-test. (e) Fold change in the number of ALDH ${ }^{\text {hi }}$ cells in SOX9 overexpressing cells over control measured by Aldefluor assay, combined results from 4 independent experiments $(n=17), p=0.004$, two-tailed paired t-test. (f) Expression levels of stem cell markers were examined by RT-qPCR, $n=6-9, p<0.05$ according to Bonferroni post hoc analysis following 2-way ANOVA, GFP control vs SOX9 overexpression. n.s. not significant. Data are shown as mean \pm SEM. 


\section{Tables}

Table 1. Physicochemical properties of SWCNTs used in this study.

\begin{tabular}{|l|c|l|}
\hline Characteristic & Value & \multicolumn{1}{|c|}{ Method used for characterization } \\
\hline $\begin{array}{l}\text { \% carbon } \\
(\mathrm{w} / \mathrm{w})\end{array}$ & $99 \%$ & $\begin{array}{l}\text { Nitric acid dissolution, inductive coupled plasma- } \\
\text { atomic emission spectrometry (ICP-AES, NMAM } \\
\# 7300)\end{array}$ \\
\hline$\% \mathrm{Fe}(\mathrm{w} / \mathrm{w})$ & $0.23 \%$ & \multicolumn{1}{|c|}{} \\
\hline Other metals & Not detectable & $\begin{array}{l}\text { Nitrogen absorption-desorption technique } \\
\text { Surface area } \\
\text { SA3100 Surface Area and Pore Size Analyzer } \\
\text { (Beckman Coulter) }\end{array}$ \\
\hline Dry length, $\mu \mathrm{m}$ & $0.1-1$ & Field emission scanning electron microscopy \\
\hline Dry width, $\mathrm{nm}$ & $0.8-1.2$ & \\
\hline
\end{tabular}




\section{References}

1. Dai, H.J. Carbon nanotubes: synthesis, integration, and properties. Acc. Chem. Res. 35, 1035-1044 (2002).

2. Popov, V.N. Carbon nanotubes: properties and application. Mat. Sci. Eng. R. 43, 61-102 (2004).

3. Poland, C.A., et al. Carbon nanotubes introduced into the abdominal cavity of mice show asbestos-like pathogenicity in a pilot study. Nat. Nanotechnol. 3, 423-428 (2008).

4. Sanchez, V.C., Pietruska, J.R., Miselis, N.R., Hurt, R.H. \& Kane, A.B. Biopersistence and potential adverse health impacts of fibrous nanomaterials: what have we learned from asbestos? WIREs Nanomed. Nanobiotechnol. 1, 511-529 (2009).

5. Lam, C.W., James, J.T., McCluskey, R. \& Hunter, R.L. Pulmonary toxicity of single-wall carbon nanotubes in mice 7 and 90 days after intratracheal instillation. Toxicol. Sci. 77, 126-134 (2004).

6. Shvedova, A.A., et al. Unusual inflammatory and fibrogenic pulmonary responses to single-walled carbon nanotubes in mice. Am. J. Physiol. Lung Cell Mol. Physiol. 289, L698-L708 (2005).

7. Shacter, E. \& Weitzman, S.A. Chronic inflammation and cancer. Oncology (Williston Park). 16, 217-226, 229; discussion 230-212 (2002).

8. Fillon, M. Details linking chronic inflammation and cancer continue to emerge. J. Natl. Cancer Inst. 105, 509-510 (2013).

9. Nagai, $\mathrm{H}$., et al. Diameter and rigidity of multiwalled carbon nanotubes are critical factors in mesothelial injury and carcinogenesis. Proc. Natl. Acad. Sci. U.S.A. 108, E1330-1338 (2011).

10. Shvedova, A.A., et al. MDSC and TGFbeta are required for racilitation of tumor growth in the lungs of mice exposed to carbon nanotubes. Cancer Res. 75, 1615-1623 (2015).

11. Suzui, M., et al. Multiwalled carbon nanotubes intratracheally instilled into the rat lung induce development of pleural malignant mesothelioma and lung tumors. Cancer Sci. 107, 924-935 (2016).

12. Kasai, T., et al. Lung carcinogenicity of inhaled multi-walled carbon nanotube in rats. Part. Fibre Toxicol. 13, 53 (2016).

13. Grosse, Y., et al. Carcinogenicity of fluoro-edenite, silicon carbide fibres and whiskers, and carbon nanotubes. The Lancet Oncology. 15, 1427-1428 (2014).

14. Wang, L., et al. Carbon nanotubes induce malignant transformation and tumorigenesis of human lung epithelial cells. Nano Lett. 11, 2796-2803 (2011).

15. Wang, L., et al. Neoplastic-like transformation effect of single-walled and multi-walled carbon nanotubes compared to asbestos on human lung small airway epithelial cells. Nanotoxicology. 8, 485-507 (2014).

16. Carel, R., et al. Occupational exposure to asbestos and man-made vitreous fibres and risk of lung cancer: a multicentre case-control study in Europe. Occup. Environ. Med. 64, 502-508 (2007).

17. Donaldson, K., Murphy, F.A., Duffin, R. \& Poland, C.A. Asbestos, carbon nanotubes and the pleural mesothelium: a review of the hypothesis regarding the role of long fibre retention in the parietal pleura, inflammation and mesothelioma. Part. Fibre Toxicol. 7(2010).

18. Pintos, J., Parent, M.-E., Rousseau, M.-C., Case, B.W. \& Siemiatycki, J. Occupational exposure to asbestos and man-made vitreous fibers, and risk of lung cancer: evidence from two case-control studies in Montreal, Canada. J. Occup. Environ. Med. 50, 12731281 (2008). 
19. Luanpitpong, S., Wang, L., Castranova, V. \& Rojanasakul, Y. Induction of stem-like cells with malignant properties by chronic exposure of human lung epithelial cells to singlewalled carbon nanotubes. Part. Fibre Toxicol. 11, 22 (2014).

20. Luanpitpong, S., et al. Caveolin-1 regulates lung cancer stem-like cell induction and p53 inactivation in carbon nanotube-driven tumorigenesis. Oncotarget. 5, 3541-3554 (2014).

21. Kreso, A. \& Dick, J.E. Evolution of the Cancer Stem Cell Model. Cell Stem Cell. 14, 275291 (2014).

22. Beck, B. \& Blanpain, C. Unravelling cancer stem cell potential. Nat. Rev. Cancer. 13, 727-738 (2013).

23. Charafe-Jauffret, E., et al. Aldehyde dehydrogenase 1-positive cancer stem cells mediate metastasis and poor clinical outcome in inflammatory breast cancer. Clin. Cancer Res. 16, 45-55 (2010).

24. Jo, A., et al. The versatile functions of Sox9 in development, stem cells, and human diseases. Genes Dis. 1, 149-161 (2014).

25. Rockich, B.E., et al. Sox9 plays multiple roles in the lung epithelium during branching morphogenesis. Proc. Natl. Acad. Sci. U.S.A. 110, E4456-4464 (2013).

26. Matheu, A., et al. Oncogenicity of the developmental transcription factor Sox9. Cancer Res. 72, 1301-1315 (2012).

27. Zhou, C.H., et al. Clinical significance of SOX9 in human non-small cell lung cancer progression and overall patient survival. J. Exp. Clin. Cancer Res. 31(2012).

28. Jiang, S.S., et al. Upregulation of SOX9 in Lung Adenocarcinoma and Its Involvement in the Regulation of Cell Growth and Tumorigenicity. Clin. Cancer Res. 16, 4363-4373 (2010).

29. Guo, W., et al. Slug and Sox9 cooperatively determine the mammary stem cell state. Cell. 148, 1015-1028 (2012).

30. Sun, L., et al. Epigenetic regulation of SOX9 by the NF-kappaB signaling pathway in pancreatic cancer stem cells. Stem Cells. 31, 1454-1466 (2013).

31. Deng, W., et al. SOX9 inhibits beta-TrCP-mediated protein degradation to promote nuclear GLI1 expression and cancer stem cell properties. J. Cell Sci. 128, 1123-1138 (2015).

32. Luanpitpong, S., et al. SLUG is required for SOX9 stabilization and functions to promote cancer stem cells and metastasis in human lung carcinoma. Oncogene. 35, 2824-2833 (2016).

33. Hanahan, D. \& Weinberg, R.A. The hallmarks of cancer. Cell. 100, 57-70 (2000).

34. Mori, S., et al. Anchorage-independent cell growth signature identifies tumors with metastatic potential. Oncogene. 28, 2796-2805 (2009).

35. Singh, S. \& Chellappan, S. Lung cancer stem cells: Molecular features and therapeutic targets. Mol. Aspects Med. 39, 50-60 (2014).

36. Ponti, D., et al. Isolation and in vitro propagation of tumorigenic breast cancer cells with stem/progenitor cell properties. Cancer Res. 65, 5506-5511 (2005).

37. Falzacappa, M.V.V., Ronchini, C., Reavie, L.B. \& Pelicci, P.G. Regulation of self-renewal in normal and cancer stem cells. FEBS J. 279, 3559-3572 (2012).

38. Hirschhaeuser, F., et al. Multicellular tumor spheroids: An underestimated tool is catching up again. J. Biotechnol. 148, 3-15 (2010).

39. Pastrana, E., Silva-Vargas, V. \& Doetsch, F. Eyes wide open: a critical review of sphereformation as an assay for stem cells. Cell Stem Cell. 8, 486-498 (2011).

40. Ginestier, C., et al. ALDH1 is a marker of normal and malignant human mammary stem cells and a predictor of poor clinical outcome. Cell Stem Cell. 1, 555-567 (2007).

41. Moreb, J.S. Aldehyde dehydrogenase as a marker for stem cells. Curr. Stem Cell Res. Ther. 3, 237-246 (2008). 
42. Marcato, P., Dean, C.A., Giacomantonio, C.A. \& Lee, P.W.K. Aldehyde dehydrogenase Its role as a cancer stem cell marker comes down to the specific isoform. Cell Cycle. 10, 1378-1384 (2011).

43. Sullivan, J.P., et al. Aldehyde dehydrogenase activity selects for lung adenocarcinoma stem cells dependent on notch signaling. Cancer Res. 70, 9937-9948 (2010).

44. Patel, M., et al. ALDH1A1 and ALDH3A1 expression in lung cancers: correlation with histologic type and potential precursors. Lung Cancer. 59, 340-349 (2008).

45. Bertolini, G., et al. Highly tumorigenic lung cancer CD133+ cells display stem-like features and are spared by cisplatin treatment. Proc Natl Acad Sci U S A. 106, 1628116286 (2009).

46. Gudas, L.J. \& Wagner, J.A. Retinoids regulate stem cell differentiation. J. Cell. Physiol. 226, 322-330 (2011).

47. Chute, J.P., et al. Inhibition of aldehyde dehydrogenase and retinoid signaling induces the expansion of human hematopoietic stem cells. Proc Natl Acad Sci U S A. 103, 11707-11712 (2006).

48. Ginestier, C., et al. Retinoid signaling regulates breast cancer stem cell differentiation. Cell Cycle. 8, 3297-3302 (2009).

49. Kopp, J.L., et al. Identification of Sox9-dependent acinar-to-ductal reprogramming as the principal mechanism for initiation of pancreatic ductal adenocarcinoma. Cancer Cell. 22, 737-750 (2012).

50. Song, S., et al. Hippo coactivator YAP1 upregulates SOX9 and endows esophageal cancer cells with stem-like properties. Cancer Res. 74, 4170-4182 (2014).

51. Luanpitpong, S., et al. Induction of stemlike cells with fibrogenic properties by carbon nanotubes and its role in fibrogenesis. Nano Lett. 14, 3110-3116 (2014).

52. Luanpitpong, S., et al. Induction of cancer-associated fibroblast-like cells by carbon nanotubes dictates its tumorigenicity. Sci. Rep. 6, 39558 (2016).

53. Shvedova, A.A., et al. Inhalation vs. aspiration of single-walled carbon nanotubes in C57BL/6 mice: inflammation, fibrosis, oxidative stress, and mutagenesis. Am. J. Physiol. Lung Cell Mol. Physiol. 295, L552-L565 (2008).

54. Pacurari, M., et al. Multi-walled carbon nanotube-induced gene expression in the mouse lung: association with lung pathology. Toxicol. Appl. Pharmacol. 255, 18-31 (2011).

55. Poulsen, S.S., et al. Transcriptomic Analysis Reveals Novel Mechanistic Insight into Murine Biological Responses to Multi-Walled Carbon Nanotubes in Lungs and Cultured Lung Epithelial Cells. PLoS One. 8(2013).

56. Chen, D., et al. Gene expression profile of human lung epithelial cells chronically exposed to single-walled carbon nanotubes. Nanoscale Res Lett. 10, 12 (2015).

57. Park, Y.-H., Kim, D., Dai, J. \& Zhang, Z. Human bronchial epithelial BEAS-2B cells, an appropriate in vitro model to study heavy metals induced carcinogenesis. Toxicol. Appl. Pharmacol. 287, 240-245 (2015).

58. Han, J.H., et al. Monitoring multiwalled carbon nanotube exposure in carbon nanotube research facility. Inhal. Toxicol. 20, 741-749 (2008).

59. Erdely, A., et al. Carbon nanotube dosimetry: from workplace exposure assessment to inhalation toxicology. Part. Fibre Toxicol. 10, 53 (2013). 


\section{Supplementary materials}

\section{Figure S1}

a

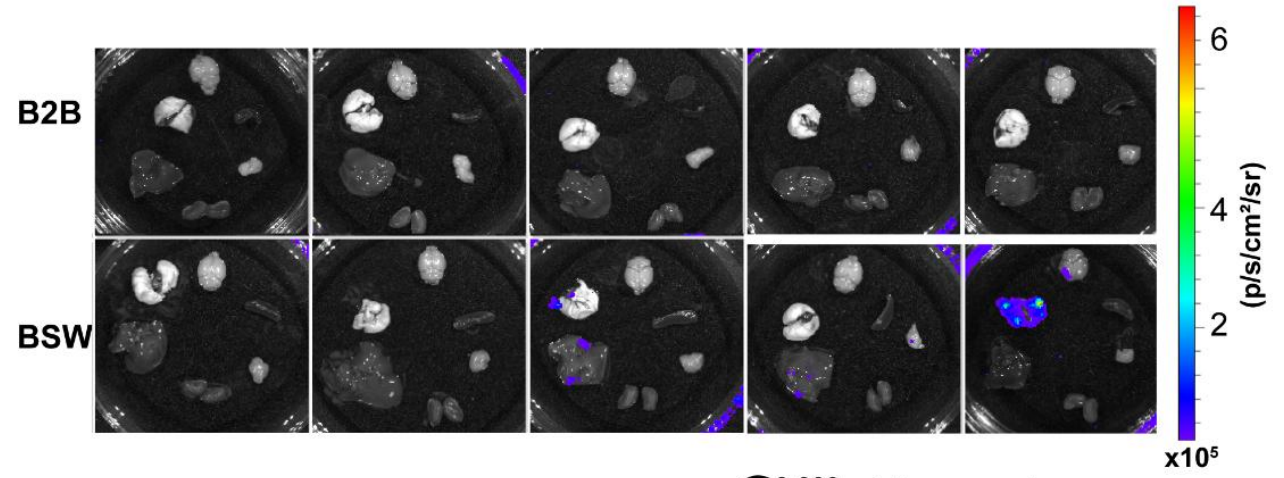

b

\begin{tabular}{|cc|}
\hline \multicolumn{2}{|c|}{ Brain } \\
Lungs & Spleen \\
& \\
Liver & Pancreas \\
Kidneys \\
\hline
\end{tabular}

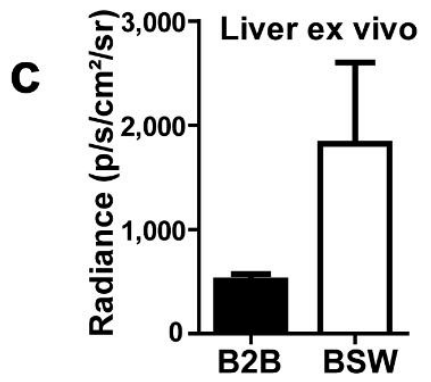

Supplementary Figure 1. BSW cells spontaneously metastasize in the subcutaneous mouse xenograft. Luciferase-labeled cells were injected subcutaneously into flanks of NOD/SCID gamma mice ( $\mathrm{n}=5$ per group). (a) Ex vivo bioluminescent signal from isolated mouse organs at the end of the experiment at week 4. (b) Schematic representation of organ positions in a. (c) Ex vivo signal from liver. Error bars are mean \pm SEM. 
a

\section{Figure S2}
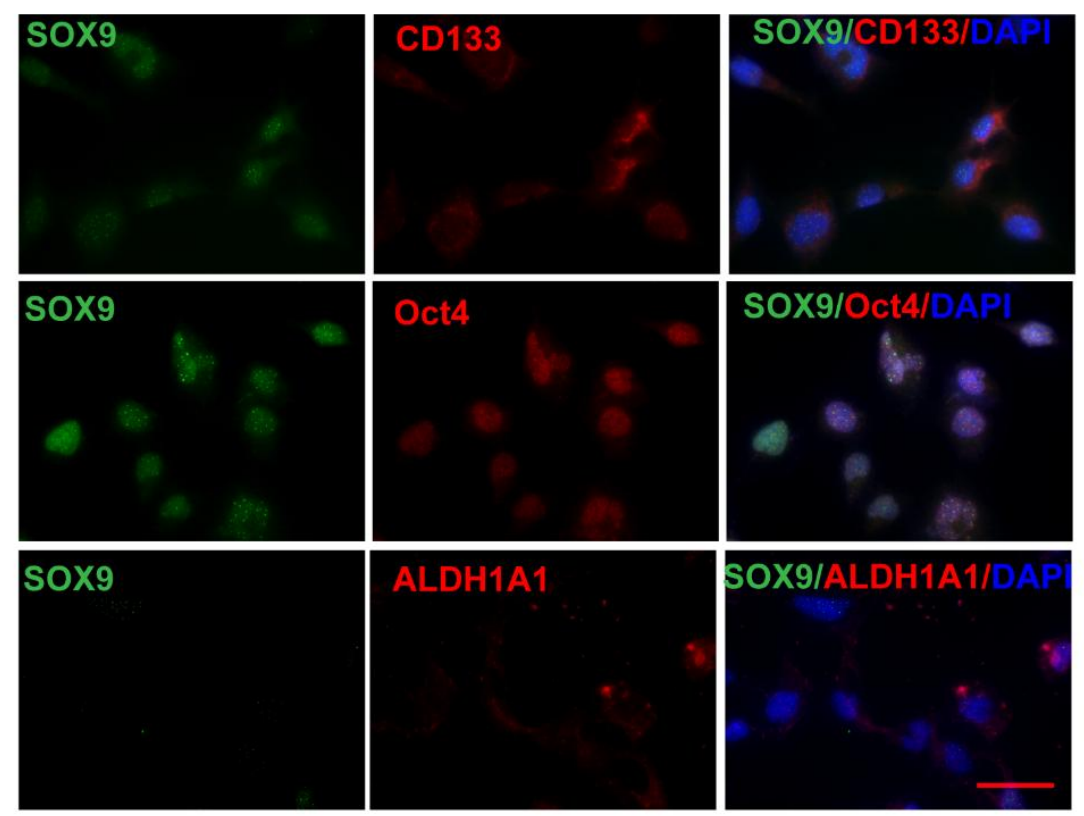

ALDH1A1

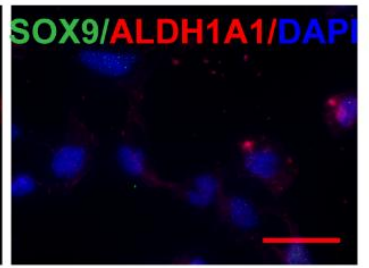

b
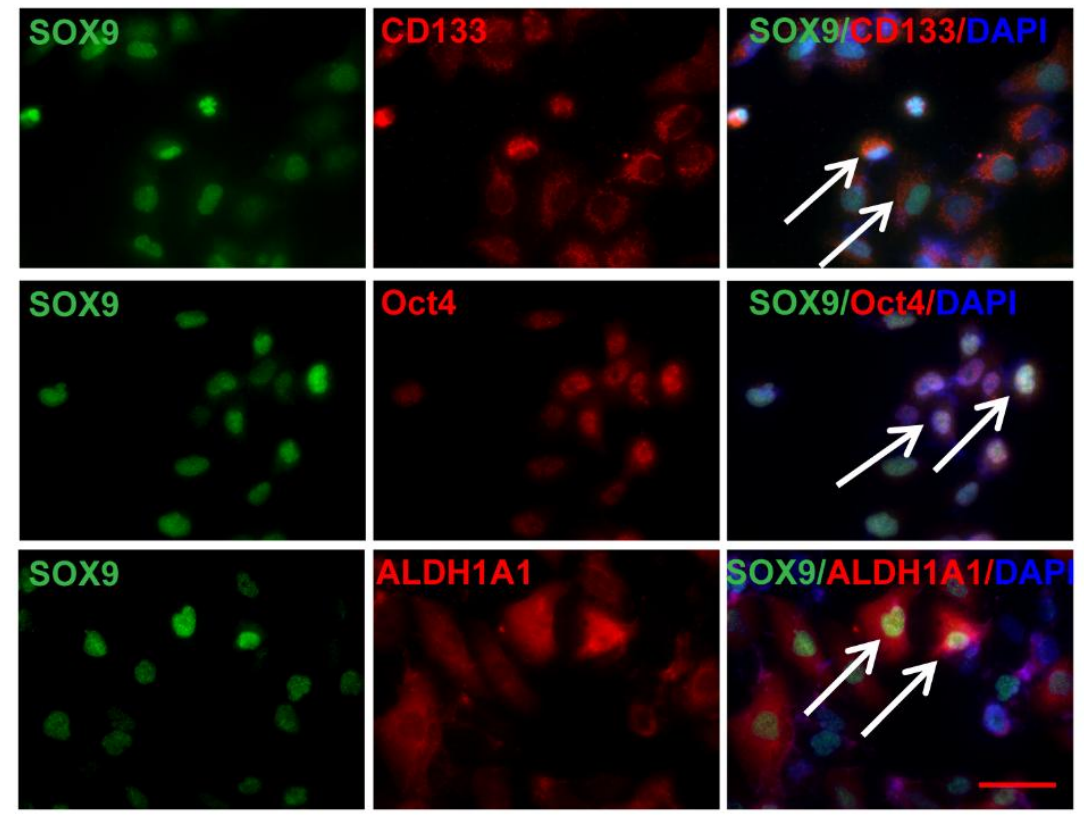

Supplementary Figure 2. SOX9 colocalizes with CSC markers. Immunofluorescence staining of CSC markers in SWCNT-treated and control cells. (a) Control Beas-2B cells, (b) BSW cells. Arrows specify cells expressing high levels of SOX9 and indicated CSC markers. Scale bar, $50 \mu \mathrm{m}$. 


\section{Figure S3}
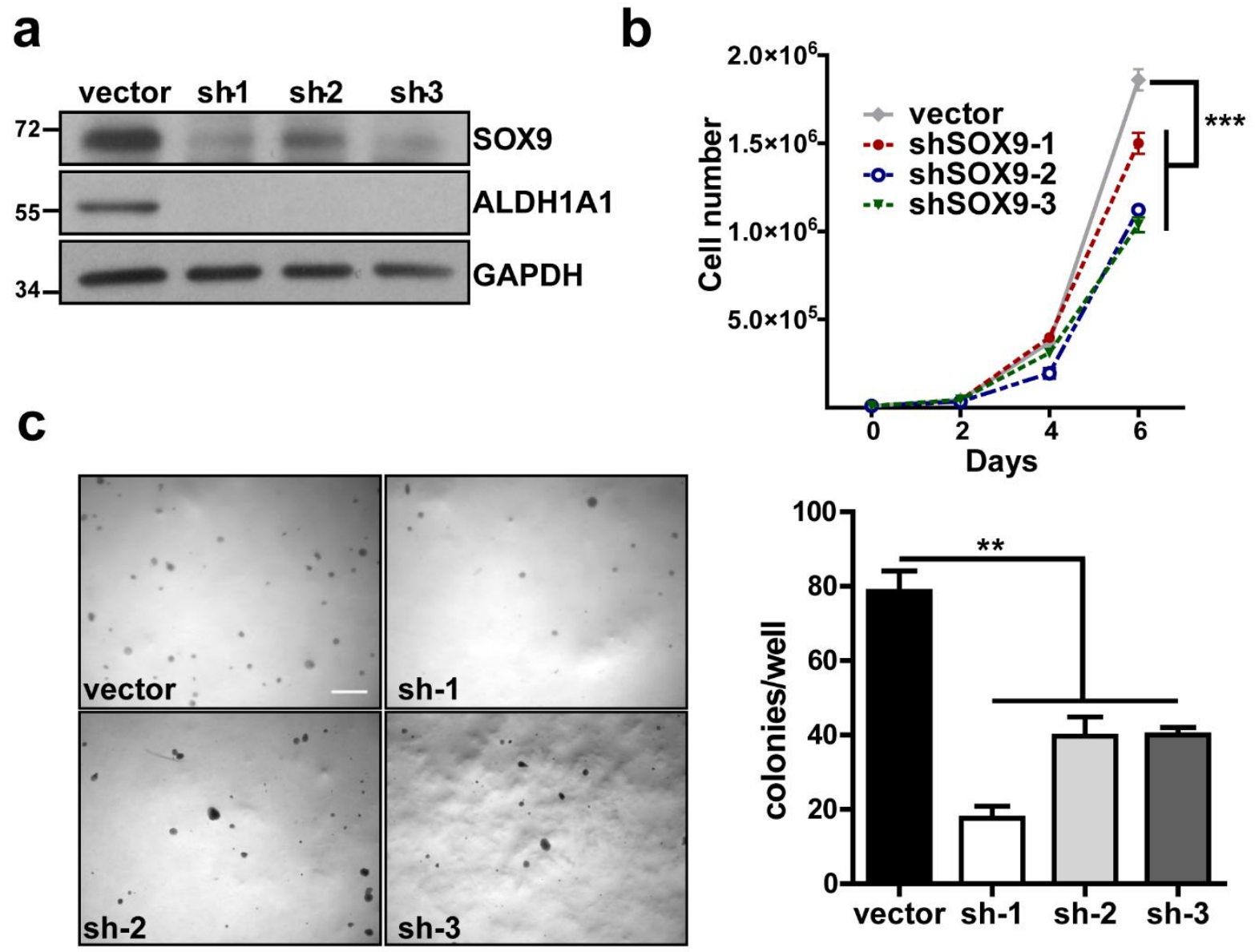

Supplementary Figure 3. SOX9 knockdown in H460 cells. (a) SOX9 knockdown by 3 shRNAs diminishes ALDH1A1 expression. (b) Cell proliferation assay, $n=2$. $P<0.001$, 2way repeated measures ANOVA followed by Bonferroni posttests, shRNAs vs vector control. (c) Soft agar colony formation. $p<0.05$, Turkey post hoc analysis following ANOVA, $\mathrm{n}=3$. Scale bar, $2 \mathrm{~mm}$. 
Supplementary Table 1. List of antibodies used in this study.

\begin{tabular}{|l|l|l|l|}
\hline Name & Company & Catalog No & Application \\
\hline SOX9 & EMD Millipore & AB5535 & WB, IHC \\
\hline SOX9 & EMD Millipore & MABC785 & IF \\
\hline ALDH1A1 & Abcam & ab52492 & WB \\
\hline ALDH1A1 & EMD Millipore & MABN838 & WB \\
\hline ALDH1A1 & Cell Signaling & 54135 & IF \\
\hline$\beta$-actin & Sigma-Aldrich & A5441 & WB \\
\hline GAPDH & EMD Millipore & CB1001 & WB \\
\hline anti-rabbit-HRP & Jackson ImmunoResearch & $711-035-152$ & WB \\
\hline anti-mouse-HRP & Jackson ImmunoResearch & $715-035-151$ & WB \\
\hline Human mitochondria & EMD Millipore & MAB1273 & IHC \\
\hline CD133 & Cell Signaling & 86781 & IF \\
\hline Oct4 & Abcam & ab19857 & IF \\
\hline
\end{tabular}

WB - western blotting, IHC - immunohistochemistry, IF - immunofluorescence.

Supplementary Table 2. Oligonucleotides for real-time quantitative PCR analysis.

\begin{tabular}{|l|l|l|}
\hline Name & Forward (5' to $\mathbf{3}^{\prime}$ ) & Reverse (5' to $\mathbf{3}^{\prime}$ ) \\
\hline ALDH1A1 & AGTGTGGGTGAATTCTATGG & TGTGACTGTTTGACCTCTGT \\
\hline ALDH1A3 & CTTCTGCCTTAGAGTCTGGAAC & TGCTACGTGGACAAGAACTG \\
\hline GAPDH & CCCACTCCTCCACCTTTGAC & ATGAGGTCCACCACCCTGTT \\
\hline CD133 & TGAATAGCAACCCTGAACTGAG & TTGGCGTTGTACTCTGTCAG \\
\hline Oct4 & TCTTCAGGAGATATGCAAAGCAGA & GATCTGCTGCAGTGTGGGT \\
\hline SOX2 & CACTGCCCCTCTCACAC & TCCATGCTGTTTCTTACTCTCC \\
\hline Nanog & GAAATACCTCAGCCTCCAGC & GCGTCACACCATTGCTATTC \\
\hline
\end{tabular}




\section{Chapter 4: General discussion and future directions}

The work presented in this dissertation provides an insight into the functional regulation of cancer cell survival during exposure to chemotherapeutic agents and nanomaterials. We demonstrated here that SOX9 expression is upregulated in NSCLC cells over the course of cisplatin treatment. Elevated SOX9 confers drug resistance by stimulating CSCs and upregulating the expression of a detoxifying enzyme and CSC marker ALDH1A1. The effect of high SOX9 expression can be blocked, at least in part, by pharmacological inhibition of ALDH1A1. These results suggest that activation of SOX9-ALDH axis in response to chemotherapy supports CSC survival. As many signaling pathways are known to activate SOX9 in embryogenesis and cancer initiation and/or progression (reviewed in Chapter 1), the exact mechanisms leading to SOX9 upregulation by chemotherapy remain to be further elucidated. If such pathways are identified, targeting these in conjunction with conventional chemotherapy may present as an attractive treatment strategy that could overcome chemoresistance. It is worth noting that a short-term exposure to cisplatin (3 days) was used as a model of aquired resistance in this study and therefore may have limited clinical relevance as cancer patients usually receive several cycles of chemotherapy. However, an extended treatment with cytotoxic agents may lead to accumulation of novel mutations, particularly in artificial cell culture conditions, and therefore mask initial mechanisms of cellular adaptation. In addition, similar results were obtained in Chapter 3 where a longterm treatment with carbon nanotubes induced neoplastic-like transformation and

chemoresistance (1) concurrent with an elevated SOX9 expression in human lung epithelial cells, suggesting that the activation of SOX9-ALDH axis may have a broader role in the regulation of cellular stress responses.

Interestingly, SOX9 also contributes to resistance to targeted and endocrine therapy. For example, SOX9 is upregulated in tamoxifen-resistant breast cancer cells and recurrent breast cancer tumors when compared to primary tumors from the same patients. Furthermore, SOX9 overexpression is sufficient to induce tamoxifen resistance in breast cancer cells (2). Additionally, SOX9 has been implicated in chemoresistance to 
a HER2 inhibitor trastuzumab in adenocarcinoma of esophagogastric junction cells (3). These results suggest that the role of SOX9 in chemotherapy resistance observed in the current work could potentially be generalized to other forms of cancer and therapeutic interventions including targeted drug therapy.

The results presented in Chapter 3 complement the Chapter 2 findings utilizing a model of particle-induced carcinogenesis. Lung cancer is often caused by environmental exposure to airborne carcinogens, with cigarette smoking being the leading risk factor (4). However, air pollution and occupational exposure have also been attributed to the high incidence of lung cancer (5). With declining rates of tobacco use, focus of research in lung cancer should be switched or expanded to less common and emerging causes as they will probably be prevalent in the future. Consistently, as new technologies are being developed and utilized, we should anticipate new kinds of carcinogens to arise. Engineered nanomaterials represent one of those new materials that are the potential source of environmental and occupational exposure to airborne carcinogens. Nanomaterials are defined as substances typically between 1 and $100 \mathrm{~nm}$ in one dimension and can be naturally occurring or man-made (engineered). Engineered nanomaterials possess novel and unique physicochemical properties that provide technological advantages over conventional materials, but they can also harbor unknown adverse health effects, including chronic diseases such as cancer. Carbon nanotubes (CNTs) are one of the widely used engineered nanomaterials due to their unique properties, such as high durability and strength, extreme light weight and high electrical conductivity; however, questions about their carcinogenicity have been raised (6). Our group demonstrated previously that a long-term exposure to low doses of CNTs induces neoplastic-like transformation in bronchial and small airway epithelial cells (7, 8). Single-walled carbon nanotube (SWCNT)-exposed Beas-2B cells exhibit elevated migration and invasion, anchorage-independent growth and form tumors in immunocompromised mice (8). Furthermore, these cells possess a highly tumorigenic and chemoresistant CSC population, while caveolin-1 was shown to regulate cell aggressiveness in vitro and in vivo $(1,9)$. 
Nevertheless, metastatic properties of SWCNT-exposed lung cells are not known and were evaluated in this dissertation for the first time. In Chapter 3, we demonstrated that SOX9 expression is upregulated in lung epithelial cells chronically exposed to SWCNT, a model mimicking a long-term occupational and environmental exposure to engineered nanomaterials. We identified SOX9 as a critical player in promoting the metastatic and stem-like properties of these cells. We also observed a correlation between SOX9 levels and ALDH expression and activity. Taken together, the findings in both chapters support the versatile role of SOX9 in regulating cell survival after exposure to various toxic agents.

CSCs have been implicated in cancer dissemination, drug resistance and recurrence (10). Thus, it is critical to elucidate CSC-specific signaling pathways and biomarkers. Unfortunately, CSCs represent a highly plastic cell population that is extremely difficult to study and, more importantly, to target for therapeutic intervention. Furthermore, CSCs are often identified and isolated based on the same markers that are expressed in normal adult stem cells. Hence, it not surprising that multiple efforts to target CSCs in clinical settings have failed (11). The results presented in this dissertation identify one of the pathways in CSC activation under the specific conditions of short-term cisplatin treatment and long-term carbon nanotube exposure. High SOX9 expression in these situations may be used as a marker for functional activation of CSCs to identify high risk populations or patients who might benefit from CSC-targeted therapy.

Interestingly, most studies on SOX9 functions reported decreased cell proliferation in SOX9 knockdown cells and increased proliferation in SOX9 overexpressing cells, i.e. in prostate (12) and lung cancers (13). We similarly observed decreased cell proliferation in SOX9 knockdown cells, but an opposite effect in overexpressing cells, i.e. decreased proliferation, suggesting that an optimal SOX9 expression is required for cancer cell proliferation. This finding is supported by previous studies showing dose-dependent effects of SOX9 in melanoma and colorectal cancer (14-16). 
With regards to mechanistic studies, we focused our attention on the functional linkage of ALDH1A1 and drug response as well as CSC properties since some information on this enzyme function was already available but required more in-depth investigation. Moreover, little is known about the role of other ALDH isoforms in cancer, and the biological functions of some isozymes are virtually unknown (17) (Table 3 in Chapter 1). Interestingly, several ALDH enzymes share preferred substrates and exhibit some degree of functional redundancy (18). Thus, ALDH1A1 may not be the only isoform involved in chemoresistance or under the regulation of SOX9. Future studies could include identification of specific ALDH isoforms contributing to drug resistance of NSCLC. This could be achieved by screening drug-resistant cells for the expression of all ALDH isoforms and confirming the results by knocking the potential candidate proteins down individually to evaluate changes in drug responses. However, this approach has several limitations. First, a knockdown of one ALDH isoform may not have a profound effect on drug sensitivity due to redundancy between these isozymes $(19,20)$. Second, it is difficult to evaluate enzymatic activity of every isoform due to the lack of specific inhibitors and assessment methods, while the enzyme expression levels may not serve as a precise measurement of their functionality. In fact, ALDH1A1 activity was shown to be regulated post-transcriptionally by acetylation (21) and phosphorylation (22). Current methods for measuring cellular ALDH activity include flow cytometry-based Aldefluor ${ }^{\circledR}$ assay (23) and spectrophotometric quantification of NADH accumulation (24); however, both techniques are not isoform-specific. Aldefluor ${ }^{\circledR}$ relies on an ALDH inhibitor DEAB which inhibits several ALDH isoforms $(25,26)$. Zhou et al. previously showed that 9 out 19 human ALDH enzymes can contribute to Aldefluor $^{\circledR}$ results, including ALDH1A1, ALDH1A2, ALDH1A3, ALDH1B1, ALDH2, ALDH3A1, ALDH3A2, ALDH3B1, and ALDH5A1 (27). While several selective ALDH1A1 inhibitors have been reported recently (28-30), the situation with specific inhibitors for other isozymes remains unresolved. Nevertheless, a pan-ALDH1 inhibitor showed promising results in preclinical models of ovarian cancer (31). Disulfiram, a drug widely used in alcoholism treatment, inhibits ALDH1A1, ALDH2, and several CSC signaling pathways (32), promoted overall survival (10 vs. 7.1 months) when administered with chemotherapy in a small phase Ilb clinical trial in patients with metastatic NSCLC (33). 
Therefore, identification of cancer type-specific ALDH isoforms contributing to drug resistance and the corresponding inhibitors could lead to more effective and personalized treatments with reduced side effects.

Another avenue for clinical applications of this work would be targeting K-rasmutated tumors. It was demonstrated that oncogenic K-ras upregulates SOX9 expression and activity in pancreatic ductal adenocarcinoma (PDAC) (34). Additionally, SOX9 expression is increased in K-ras mutated lung adenocarcinomas and in a Kras $^{\text {G12D }}$ mouse model for lung adenocarcinoma (35). K-ras driver-mutations are currently not druggable by targeted therapy but are identified in up to $25 \%$ of human lung adenocarcinomas (36), hence, pharmacologic intervention at the level of SOX9 and/or ALDH may be a promising treatment strategy to pursue.

Further studies are also needed to evaluate how broadly SOX9 activates the CSC survival program. ChIP-seq would a logical next step to identify additional proteins participating in this program. However, only a few direct transcriptional targets of SOX9 have been identified thus far, probably because SOX9 operates in collaboration with other factors to regulate transcription, and because this cooperation may be tissue specific or context dependent $(37,38)$. Hence, more studies are warranted to identify the molecular machinery governing CSC survival under these cellular stress conditions.

The current work investigated the molecular mechanisms of CSC regulation under drug and particle-induced cellular stress using established lung cell lines. Additional studies in other cell systems and in vivo will be needed to validate the results. To that end, mice bearing NSCLC cells or patient derived tissues can be exposed to chemotherapy drugs and then tumors analyzed for SOX9 expression. Chemotherapy treatment in combination with an ALDH inhibitor could be used to validate our in vitro results. However, most available ALDH inhibitors are not entirely specific to ALDH1A1 but also affect other isoforms, although several new isozyme-specific inhibitors are being developed and have been reported $(30,39,40)$.

However, xenograft mouse models do not fully recapitulate all aspects of tumorigenesis, including the initial stages of development and contributions from the 
tumor microenvironment. Several existing transgenic mouse models could be used to overcome this limitation. For instance, Lox-Stop-Lox K-ras conditional mouse strain (LSL-K-ras ${ }^{\mathrm{G} 12 \mathrm{D}}$ ), in which the expression of endogenous levels of oncogenic $\mathrm{K}$-ras is controlled by a removable transcriptional termination stop element, forms adenomas and adenocarcinomas (41). SOX9 can be removed conditionally by breeding K-ras ${ }^{\mathrm{G} 12 \mathrm{D}}$ with Sox $9^{\text {floxflox }}$ mice (42). SOX9 deletion can be performed at the same time as activation of oncogenic K-ras through the administration of an engineered Adeno-Cre virus via intratracheal instillation. I expect a delayed and/or reduced tumor initiation in this mouse model but SOX9 removal may not be sufficient to completely inhibit tumorigenesis due to functional redundancy in oncogenic signaling pathways. Similar results were observed in PDAC where malignant lesions were formed only from cells that retained SOX9 expression due to limitations in recombination efficiency (43). One of the possible ways to avoid incomplete transgene recombination is to generate SOX9 knockout in LSL-K-ras ${ }^{\text {G12D }}$ mice by lentivirus transduction. CRISPR/Cas9-sgSOX9 lentivirus delivered through intratracheal administration would induce double-strand DNA breaks in the same lung cells that undergo activation of the mutant Kras.

LSL-K-ras ${ }^{\mathrm{G} 12 \mathrm{D}}$ model is widely used to generate early stage cancer, however, mouse models for late stage and metastatic lung cancer would be more clinically relevant as the majority of NSCLC patients are diagnosed when cancer has already metastasized (44). LSL-K-ras ${ }^{\mathrm{G} 12 \mathrm{D}}$; p53 ${ }^{\mathrm{Flox}}$ mice develop advanced adenocarcinomas with local invasion and metastases to lymph nodes and distant organs $(45,46)$. CRISPR/Cas9-sgSOX9 lentivirus delivered intratracheally to these animals is supposed to reduce tumor formation and metastasis as compared to mice received sgControl virus. Another interesting use of the same model is to investigate metastatic properties of SOX9 without affecting primary tumors. In this case, LSL-K-ras ${ }^{\mathrm{G} 12 \mathrm{D}}$; $\mathrm{p} 53^{\mathrm{Flox}}$ mice can be crossed with CCSP-rtTA/(teto) ${ }_{7}-\mathrm{CMV}$-shSOX9 mice (not available yet to my knowledge), where the reverse tetracycline trans-activator protein (rtTA) is expressed under Clara cell secretory protein (CCSP) promoter (47). Therefore, activation of the mutant Kras and p53 deletion will be stimulated by Adeno-Cre virus via an intratracheal instillation, allowing for primary tumor formation. SOX9 knockdown can be induced by providing mice with doxycycline-containing food at different time points after the 
induction of primary tumors. These experiments would shed light on the role of SOX9 in the initiation of metastasis and would elucidate SOX9-dependent metastatic events and their timing. SOX9 overexpression experiments could be performed in the same lung cancer models to support the results of SOX9 knockout. Overexpression studies could be accomplished by using SOX9 knockin mice CAG-mRFP1 ${ }^{\text {flox }}$-Sox9-EGFP (CAGSox9) (48). Doxycycline-inducible lentiviral particles with SOX9 expression constructs can be used if we want to disconnect the timing of tumor initiation and SOX9 overexpression.

In summary, this dissertation work identified SOX9 as a critical regulator of CSC survival in drug and particle-exposed human lung cells, and provided evidence that it acts, at least in part, through transcriptional activation of ALDH1A1. The SOX9-ALDH axis could have a broad role in regulating CSC survival under diverse cellular stress conditions and in various cancer types. More studies are still needed to evaluate the upstream signaling pathways and other downstream effectors of SOX9 for better understanding of its regulatory mechanisms and potential therapeutic applications. 


\section{References}

1. Luanpitpong S, Wang L, Castranova V, Rojanasakul Y. Induction of stem-like cells with malignant properties by chronic exposure of human lung epithelial cells to single-walled carbon nanotubes. Part Fibre Toxicol. 2014;11:22.

2. Jeselsohn R, Cornwell M, Pun M, Buchwalter G, Nguyen M, Bango C, et al. Embryonic transcription factor SOX9 drives breast cancer endocrine resistance. Proc Natl Acad Sci USA. 2017;114(22):E4482-E91.

3. Hong $\mathrm{Y}$, Chen $\mathrm{H}$, Rao Z, Peng $\mathrm{B}, \mathrm{Hu} \mathrm{H}$, Lin S, et al. In vitro study on the role of SOX9 in trastuzumab resistance of adenocarcinoma of the esophagogastric junction. Exp Ther Med. 2018;15(3):3103-7.

4. $\quad$ American Cancer Society. Cancer Facts \& Figures 2019. 2019.

5. Malhotra J, Malvezzi M, Negri E, La Vecchia C, Boffetta P. Risk factors for lung cancer worldwide. Eur Respir J. 2016;48(3):889-902.

6. Donaldson K, Poland CA, Murphy FA, MacFarlane M, Chernova T, Schinwald A. Pulmonary toxicity of carbon nanotubes and asbestos - similarities and differences. Adv Drug Deliv Rev. 2013;65(15):2078-86.

7. Wang L, Stueckle TA, Mishra A, Derk R, Meighan T, Castranova V, et al. Neoplastic-like transformation effect of single-walled and multi-walled carbon nanotubes compared to asbestos on human lung small airway epithelial cells. Nanotoxicology. 2014;8(5):485-507.

8. Wang L, Luanpitpong S, Castranova V, Tse W, Lu Y, Pongrakhananon V, et al. Carbon nanotubes induce malignant transformation and tumorigenesis of human lung epithelial cells. Nano Lett. 2011;11(7):2796-803.

9. Luanpitpong S, Wang L, Stueckle TA, Tse W, Chen YC, Rojanasakul Y. Caveolin-1 regulates lung cancer stem-like cell induction and p53 inactivation in carbon nanotube-driven tumorigenesis. Oncotarget. 2014;5(11):3541-54.

10. Clarke MF, Fuller M. Stem cells and cancer: two faces of eve. Cell. 2006;124(6):1111-5.

11. Batlle E, Clevers H. Cancer stem cells revisited. Nat Med. 2017;23(10):1124-34.

12. Thomsen MK, Ambroisine L, Wynn S, Cheah KSE, Foster CS, Fisher G, et al. SOX9 elevation in the prostate promotes proliferation and cooperates with PTEN loss to drive tumor formation. Cancer Res. 2010;70(3):979-87.

13. Wang X, Ju Y, Zhou MI, Liu X, Zhou C. Upregulation of SOX9 promotes cell proliferation, migration and invasion in lung adenocarcinoma. Oncol Lett. 2015;10(2):990-4.

14. Yang X, Liang R, Liu C, Liu JA, Cheung MPL, Liu X, et al. SOX9 is a dose-dependent metastatic fate determinant in melanoma. Journal of experimental \& clinical cancer research : CR. 2019;38(1):17.

15. Prevostel C, Rammah-Bouazza C, Trauchessec H, Canterel-Thouennon L, Busson M, Ychou M, et al. SOX9 is an atypical intestinal tumor suppressor controlling the oncogenic Wnt/SS-catenin signaling. Oncotarget. 2016;7(50):82228-43.

16. Prevostel C, Blache P. The dose-dependent effect of SOX9 and its incidence in colorectal cancer. European journal of cancer (Oxford, England : 1990). 2017;86:150-7.

17. Tomita H, Tanaka K, Tanaka T, Hara A. Aldehyde dehydrogenase $1 \mathrm{~A} 1$ in stem cells and cancer. Oncotarget. 2016;7(10):11018-32.

18. Marchitti SA, Brocker C, Stagos D, Vasiliou V. Non-P450 aldehyde oxidizing enzymes: the aldehyde dehydrogenase superfamily. Expert Opin Drug Metab Toxicol. 2008;4(6):697-720.

19. Moreb JS, Baker HV, Chang LJ, Amaya M, Lopez MC, Ostmark B, et al. ALDH isozymes downregulation affects cell growth, cell motility and gene expression in lung cancer cells. Mol Cancer. 2008;7:87.

20. Moreb JS, Mohuczy D, Muhoczy D, Ostmark B, Zucali JR. RNAi-mediated knockdown of aldehyde dehydrogenase class-1A1 and class-3A1 is specific and reveals that each contributes equally to the resistance against 4-hydroperoxycyclophosphamide. Cancer Chemother Pharmacol. 2007;59(1):127-36. 
21. Zhao D, Mo Y, Li MT, Zou SW, Cheng ZL, Sun YP, et al. NOTCH-induced aldehyde dehydrogenase $1 \mathrm{~A} 1$ deacetylation promotes breast cancer stem cells. J Clin Invest. 2014;124(12):5453-65.

22. Wang J, Nikhil K, Viccaro K, Chang L, White J, Shah K. Phosphorylation-dependent regulation of ALDH1A1 by Aurora kinase $A$ : insights on their synergistic relationship in pancreatic cancer. BMC Biol. 2017;15(1):10.

23. Storms RW, Trujillo AP, Springer JB, Shah L, Colvin OM, Ludeman SM, et al. Isolation of primitive human hematopoietic progenitors on the basis of aldehyde dehydrogenase activity. Proc Natl Acad Sci U S A. 1999;96(16):9118-23.

24. Moreb JS, Maccow C, Schweder M, Hecomovich J. Expression of antisense RNA to aldehyde dehydrogenase class-1 sensitizes tumor cells to 4-hydroperoxycyclophosphamide in vitro. The Journal of pharmacology and experimental therapeutics. 2000;293(2):390-6.

25. Moreb JS, Ucar D, Han S, Amory JK, Goldstein AS, Ostmark B, et al. The enzymatic activity of human aldehyde dehydrogenases $1 \mathrm{~A} 2$ and 2 (ALDH1A2 and ALDH2) is detected by Aldefluor, inhibited by diethylaminobenzaldehyde and has significant effects on cell proliferation and drug resistance. Chem Biol Interact. 2012;195(1):52-60.

26. Luo M, Gates KS, Henzl MT, Tanner JJ. Diethylaminobenzaldehyde is a covalent, irreversible inactivator of ALDH7A1. ACS Chem Biol. 2015;10(3):693-7.

27. Zhou L, Sheng D, Wang D, Ma W, Deng Q, Deng L, et al. Identification of cancer-type specific expression patterns for active aldehyde dehydrogenase (ALDH) isoforms in ALDEFLUOR assay. Cell Biol Toxicol. 2018.

28. Condello S, Morgan CA, Nagdas S, Cao L, Turek J, Hurley TD, et al. beta-Cateninregulated ALDH1A1 is a target in ovarian cancer spheroids. Oncogene. 2015;34(18):2297-308.

29. Morgan CA, Hurley TD. Characterization of two distinct structural classes of selective aldehyde dehydrogenase 1A1 inhibitors. J Med Chem. 2015;58(4):1964-75.

30. Yang SM, Martinez NJ, Yasgar A, Danchik C, Johansson C, Wang Y, et al. Discovery of Orally Bioavailable, Quinoline-Based Aldehyde Dehydrogenase 1A1 (ALDH1A1) Inhibitors with Potent Cellular Activity. J Med Chem. 2018;61(11):4883-903.

31. Chefetz I, Grimley E, Yang K, Hong L, Vinogradova EV, Suciu R, et al. A Pan-ALDH1A Inhibitor Induces Necroptosis in Ovarian Cancer Stem-like Cells. Cell Rep. 2019;26(11):3061-75 e6.

32. Marcucci F, Rumio C, Lefoulon F. Anti-Cancer Stem-like Cell Compounds in Clinical Development - An Overview and Critical Appraisal. Front Oncol. 2016;6:115.

33. Nechushtan H, Hamamreh Y, Nidal S, Gotfried M, Baron A, Shalev YI, et al. A phase Ilb trial assessing the addition of disulfiram to chemotherapy for the treatment of metastatic nonsmall cell lung cancer. The oncologist. 2015;20(4):366-7.

34. Zhou H, Qin Y, Ji S, Ling J, Fu J, Zhuang Z, et al. SOX9 activity is induced by oncogenic Kras to affect MDC1 and MCMs expression in pancreatic cancer. Oncogene. 2018;37(7):91223.

35. Capaccione KM, Hong X, Morgan KM, Liu W, Bishop JM, Liu L, et al. Sox9 mediates Notch1-induced mesenchymal features in lung adenocarcinoma. Oncotarget. 2014;5(11):363650 .

36. Hirsch FR, Suda K, Wiens J, Bunn PA, Jr. New and emerging targeted treatments in advanced non-small-cell lung cancer. Lancet (London, England). 2016;388(10048):1012-24.

37. Shi Z, Chiang Cl, Labhart P, Zhao Y, Yang J, Mistretta TA, et al. Context-specific role of SOX9 in NF-Y mediated gene regulation in colorectal cancer cells. Nucleic Acids Res. 2015;43(13):6257-69.

38. Ohba S, He X, Hojo H, McMahon AP. Distinct Transcriptional Programs Underlie Sox9 Regulation of the Mammalian Chondrocyte. Cell reports. 2015;12(2):229-43. 
39. Yang SM, Yasgar A, Miller B, Lal-Nag M, Brimacombe K, Hu X, et al. Discovery of NCT501, a Potent and Selective Theophylline-Based Inhibitor of Aldehyde Dehydrogenase $1 \mathrm{~A} 1$ (ALDH1A1). J Med Chem. 2015;58(15):5967-78.

40. Huddle BC, Grimley E, Buchman CD, Chtcherbinine M, Debnath B, Mehta P, et al. Structure-Based Optimization of a Novel Class of Aldehyde Dehydrogenase 1A (ALDH1A) Subfamily-Selective Inhibitors as Potential Adjuncts to Ovarian Cancer Chemotherapy. J Med Chem. 2018;61(19):8754-73.

41. Jackson EL, Willis N, Mercer K, Bronson RT, Crowley D, Montoya R, et al. Analysis of lung tumor initiation and progression using conditional expression of oncogenic K-ras. Genes Dev. 2001;15(24):3243-8.

42. Akiyama H, Chaboissier MC, Martin JF, Schedl A, de Crombrugghe B. The transcription factor Sox9 has essential roles in successive steps of the chondrocyte differentiation pathway and is required for expression of Sox5 and Sox6. J Bone Miner Res. 2002;17:S142-S.

43. Kopp JL, von Figura G, Mayes E, Liu FF, Dubois CL, Morris JPt, et al. Identification of Sox9-dependent acinar-to-ductal reprogramming as the principal mechanism for initiation of pancreatic ductal adenocarcinoma. Cancer Cell. 2012;22(6):737-50.

44. Cronin KA, Lake AJ, Scott S, Sherman RL, Noone AM, Howlader N, et al. Annual Report to the Nation on the Status of Cancer, part I: National cancer statistics. Cancer. 2018;124(13):2785-800.

45. Jackson EL, Olive KP, Tuveson DA, Bronson R, Crowley D, Brown M, et al. The differential effects of mutant p53 alleles on advanced murine lung cancer. Cancer Res. 2005;65(22):10280-8.

46. Winslow MM, Dayton TL, Verhaak RGW, Kim-Kiselak C, Snyder EL, Feldser DM, et al. Suppression of lung adenocarcinoma progression by Nkx2-1. Nature. 2011;473(7345):101-4.

47. Perl A-K, Zhang L, Whitsett JA. Conditional expression of genes in the respiratory epithelium in transgenic mice: cautionary notes and toward building a better mouse trap. Am J Respir Cell Mol Biol. 2009;40(1):1-3.

48. Kim Y, Murao H, Yamamoto K, Deng JM, Behringer RR, Nakamura T, et al. Generation of transgenic mice for conditional overexpression of Sox9. J Bone Miner Metab. 2011;29(1):1239. 